MATHEMATICS OF COMPUTATION

Volume 72, Number 243, Pages 1179-1214

S 0025-5718(02)01471-0

Article electronically published on October 18, 2002

\title{
THE $h p$-LOCAL DISCONTINUOUS GALERKIN METHOD FOR LOW-FREQUENCY TIME-HARMONIC MAXWELL EQUATIONS
}

\author{
ILARIA PERUGIA AND DOMINIK SCHÖTZAU
}

\begin{abstract}
The local discontinuous Galerkin method for the numerical approximation of the time-harmonic Maxwell equations in a low-frequency regime is introduced and analyzed. Topologically nontrivial domains and heterogeneous media are considered, containing both conducting and insulating materials. The presented method involves discontinuous Galerkin discretizations of the curl-curl and grad-div operators, derived by introducing suitable auxiliary variables and so-called numerical fluxes. An $h p$-analysis is carried out and error estimates that are optimal in the meshsize $h$ and slightly suboptimal in the approximation degree $p$ are obtained.
\end{abstract}

\section{INTRODUCTION}

In this paper, we propose and analyze an $h p$-local discontinuous Galerkin (LDG) method for the low-frequency time-harmonic Maxwell equations in heterogeneous media, containing both conducting and insulating materials: Find the complex field E that satisfies

$$
\begin{aligned}
\nabla \times\left(\mu^{-1} \nabla \times \mathbf{E}\right)+i \omega \sigma \mathbf{E} & =-i \omega \mathbf{J}_{s}=: \mathbf{J} & & \text { in } \Omega \subset \mathbb{R}^{3}, \\
\nabla \cdot(\varepsilon \mathbf{E}) & =0 & & \text { in } \Omega_{0} \subset \Omega,
\end{aligned}
$$

together with suitable boundary conditions (see Alonso and Valli 2 and Alonso 1]). Here, the field $\mathbf{E}$ is related to the electric field $\mathcal{E}$ by the identity $\mathcal{E}(\mathbf{x}, t)=$ $\operatorname{Re}\left(\mathbf{E}(\mathbf{x}) e^{i \omega t}\right)$, where $\omega \neq 0$ is a given frequency. The parameter $\mu=\mu(\mathbf{x})$ is the magnetic permeability, $\varepsilon=\varepsilon(\mathbf{x})$ the electric permittivity, $\mathbf{J}_{s}$ is the phasor associated with a given current density, and $\sigma=\sigma(\mathbf{x})$ is the electric conductivity, which is zero in the subdomain $\Omega_{0}$ occupied by insulating materials. We remark that the electric field-based formulation in (1.1)-(1.2) is only one of several field- and potential-based formulations proposed in the literature for the solution of eddy current problems

Received by the editor July 9, 2001 and, in revised form, December 10, 2001.

2000 Mathematics Subject Classification. Primary 65N30.

Key words and phrases. hp-finite elements, discontinuous Galerkin methods, low-frequency time-harmonic Maxwell equations, heterogeneous media.

The first author was supported in part by NSF Grant DMS-9807491 and by the University of Minnesota Supercomputing Institute. This work was carried out when the author was visiting the School of Mathematics, University of Minnesota.

The second author was supported in part by NSF Grant DMS-0107609 and by the University of Minnesota Supercomputing Institute. This work was carried out while the author was affiliated with the School of Mathematics, University of Minnesota. 
(see, e.g., Bryan, Emson, Fernandes and Trowbridge [13, Bossavit 12], Hiptmair [33] and the references therein).

The main motivation for using discontinuous Galerkin (DG) methods for the numerical approximation of the above problem is that these methods, being based on discontinuous finite element spaces, can easily handle meshes with hanging nodes, elements of general shape, and local spaces of different types. Thus, they are ideally suited for $h p$-adaptivity and multiphysics or multimaterial problems. This flexibility in the mesh design is not shared in a straightforward way by standard edge or face elements commonly used in computational electromagnetics. Indeed, these elements are designed to enforce the continuity of either the tangential or the normal components of the fields across interelement boundaries (see, e.g., Nédéléc 37, 38, Bossavit [10 11, and Monk 36]). This makes the handling of nonmatching grids and high-order approximations rather inconvenient from an implementational point of view. Nevertheless, efficient $h p$-adaptive edge element methods have been developed recently by Demkowicz and Vardapetyan [29, 45].

There are several possibilities for dealing with the divergence-free constraint (1.2) in the subregion $\Omega_{0}$. One way of imposing this constraint is to use mixed formulations, where new unknowns are introduced as Lagrange multipliers (see, e.g., Chen, Du and Zou [21, Demkowicz and Vardapetyan [29, 45] and the references therein). Other approaches consist in regularizing the formulation in $\Omega_{0}$ by adding suitable terms containing the divergence of $\mathbf{E}$, giving rise to formulations in the variable $\mathbf{E}$ only (see, e.g., Alonso and Valli [2] where an iteration-by-subdomain procedure is studied, using edge elements in the conducting region $\Omega \backslash \Omega_{0}$ and continuous elements in $\Omega_{0}$ ). It is well known that, if the solution exhibits corner singularities, the discretization of regularized formulations by means of continuous elements can result in discrete solutions that converge to a vector function that is not a solution of Maxwell equations. Remedies to overcome this problem have been presented in Bonnet-BenDhia, Hazard and Lohrengel [9], where a singular field method is introduced, and in Dauge, Costabel and Martin 28], where the bilinear forms are suitably weighted near solution singularities. Here we impose the divergence-free constraint by a regularization approach, but propose methods that are based on completely discontinuous spaces. This might give an alternative way to overcome some of the problems related to continuous elements. In this paper, however, we prove basic $h p$-error estimates for the proposed LDG scheme under the assumption of piece-wise smooth exact solutions.

The LDG method has been introduced by Cockburn and Shu 25 for convectiondiffusion systems, and has been further developed and analyzed in Cockburn and Dawson [23], Castillo, Cockburn, Schötzau and Schwab [20, Castillo, Cockburn, Perugia and Schötzau [19, Cockburn, Kanschat, Perugia and Schötzau 24]; see also the review by Cockburn and Shu 26]. It is one of several DG methods that have been proposed in the literature for diffusion problems. We only mention here the DG methods of Baumann and Oden [8] and Oden, Babuška and Baumann 39], and the interior penalty (IP) methods and their variants which have been recently studied; e.g., in Rivière, Wheeler and Girault [43], Rivière and Wheeler [42] and Houston, Schwab and Süli [34]. A comparison of DG methods from a computational point of view can be found in Castillo [18. Recent works have unified the presentation and the analysis of all these methods for elliptic problems. In Prudhomme, Pascal, Oden and Romkes [41, an $h p$-analysis of different DG methods has been given, including the Baumann-Oden method and interior penalty methods. Furthermore, 
in Arnold, Brezzi, Cockburn and Marini [6], a framework has been presented within which virtually all the DG methods found in the literature can be analyzed; it is based on a mixed formulation of the second-order problem and on the so-called numerical fluxes.

The LDG method for the discretization of (1.1)-(1.2) is designed by adapting to the curl-curl and grad-div operators the definition of the numerical fluxes considered in 25, 19] for the Laplacian. This is done in a consistent way and such that the auxiliary variables needed to define the LDG formulation can be eliminated from the equations in an element-by-element manner. For discontinuity stabilization parameters of the order $p^{2} / h$, we prove error estimates that are optimal in the meshsize $h$ and slightly suboptimal in the polynomial degree $p$ (half a power of $p$ is lost). This analysis is the first $h p$-error analysis for the LDG method in several space dimensions, and in this sense it extends previous work in 20, 19. For multidimensional elliptic problems on general unstructured meshes no better $p$-bounds can be found in the DG literature (see, e.g., Rivière, Wheeler and Girault [43, Prudhomme, Pascal, Oden and Romkes [41] and Houston, Schwab and Süli [34, where the same rates of convergence as in our case are obtained with different analysis techniques). We mention, however, that improved $p$-bounds have been proved by Castillo, Cockburn, Schötzau and Schwab [20] for one-dimensional convectiondiffusion problems, and recently by Georgoulis and Süli 32 for two-dimensional reaction-diffusion problems on affine quadrilateral grids containing hanging nodes.

The outline of the paper is as follows. In Section 2, we present the low-frequency time-harmonic Maxwell equations in heterogeneous media, under quite general and realistic assumptions on the domain and the data. We need to extend to our case the existence and uniqueness results established in [2] for a more particular situation. The proof of these extensions is developed in detail in the appendix, and relies on the existence of a continuous lifting of tangential traces, which is divergence-free in $\Omega_{0}$ and satisfies certain homogeneous flux conditions through the cavities of $\Omega_{0}$. In Section 3 we derive the LDG method and show that it defines a unique approximate solution. An $h p$-error analysis is carried out in Section 4 Possible extensions of our work and concluding remarks are presented in Section 5

\section{The Model PRoblem in Heterogeneous media}

In this section, we specify our assumptions on the domain and the data, and present the complete model problem in heterogeneous media. The proof of the well-posedness of the continuous problem is postponed until the appendix.

2.1. Preliminaries. We start by making precise the assumptions on the domain and the data, and by introducing the functional spaces used throughout the paper.

Assumptions on the domain. Let $\Omega$ be a connected, bounded, open Lipschitz polyhedron in $\mathbb{R}^{3}$, whose boundary may contain several connected, not necessarily simply connected components. Throughout the paper, whenever referring to a non-simply connected domain, we assume that there exists an "admissible set of cuts" in the sense of [4], whose removal reduces the domain to a simply connected one (see also [31] for further comments). Let $\Omega_{0}$ be the subdomain of $\Omega$ occupied by insulating materials. We define $\Omega_{\sigma}=\Omega \backslash \Omega_{0}$, and denote by $\Gamma$ the interface $\partial \Omega_{0} \cap \partial \Omega_{\sigma}$. We assume $\Omega_{0}$ and $\Omega_{\sigma}$ to be open Lipschitz polyhedra such that the closure of $\Gamma$ is a collection of closed faces of $\partial \Omega_{0}$ and $\partial \Omega_{\sigma}$. For the sake of 


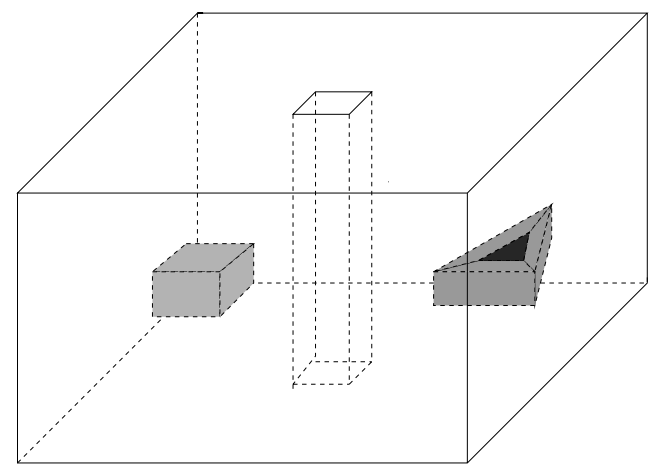

Figure 1. Example of an admissible domain: $\Omega$ is the big parallelepiped with rectangular hole ( $\partial \Omega$ has one non-simply connected component), $\Omega_{\sigma}$ is the union of the shadowed parallelepiped and prisma with triangular hole, and $\Omega_{0}=\Omega \backslash \Omega_{\sigma}$. Therefore, $\partial \Omega_{0}=\Gamma_{0,0} \cup \Gamma_{0,1} \cup \Gamma_{0,2}$, where the external component $\Gamma_{0,0}$ coincides with $\partial \Omega$, and $\Gamma_{0,1}$ and $\Gamma_{0,2}$ are boundaries of the shadowed parallelepiped and prisma, respectively $\left(\Gamma_{0,1}\right.$ is simply connected, whereas $\Gamma_{0,0}$ and $\Gamma_{0,2}$ are not).

simplicity, we assume $\Omega_{0}$ to be connected. The extension to the general case where $\Omega_{0}$ is not connected can be done easily by dealing with each of the connected components of $\Omega_{0}$ as done with $\Omega_{0}$ in this paper. Let $\Gamma_{0, j}, j=0, \ldots, J$, be the connected, not necessarily simply connected components of $\partial \Omega_{0}$. We denote by $\Gamma_{0,0}$ the "external" connected component of $\partial \Omega_{0}$, defined as the boundary of the only unbounded component of $\mathbb{R}^{3} \backslash \bar{\Omega}_{0}$, and by $\Gamma_{0, j}, j=1, \ldots, J$, the possible "cavities" of $\Omega_{0}$, which are boundaries of connected, bounded Lipschitz polyhedra in $\mathbb{R}^{3} \backslash \bar{\Omega}_{0}$. An example of an admissible domain is given in Figure 1 .

Assumptions on the data. The magnetic permeability and reluctivity $\mu$ and $\mu^{-1}$ and the electric permittivity $\varepsilon$ are symmetric, uniformly positive definite tensors with bounded coefficients. The electric conductivity $\sigma$ is a symmetric tensor with bounded coefficients, uniformly positive definite in the conducting region $\Omega_{\sigma}$ and zero in $\Omega_{0}$. These tensors are smooth within any subdomain occupied by a single material, and might be discontinuous across the interfaces between different materials. Finally, the current density $\mathbf{J}$ satisfies $\mathbf{J}=\mathbf{0}$ in $\Omega_{\sigma}, \nabla \cdot \mathbf{J}=0$ in $\Omega_{0}$ and $\mathbf{J} \cdot \mathbf{n}_{0}=0$ on $\partial \Omega_{0}$, where $\mathbf{n}_{0}$ is the outward normal unit vector to $\partial \Omega_{0}$. Moreover, if $\Omega_{0}$ is not simply connected, denoting by $\left\{\Sigma_{\ell}\right\}_{\ell=1, \ldots, L}$ an admissible set of cuts for $\Omega_{0}$, we also assume that $\mathbf{J}$ has zero flux through each $\Sigma_{\ell}$.

Functional spaces. Given a domain $D$ in $\mathbb{R}^{2}$ or $\mathbb{R}^{3}$, we denote, as usual, by $H^{s}(D)^{d}, d=1,2,3$, the Sobolev space of real or complex functions with integer or fractional regularity exponent $s \geq 0$, endowed with the norm $\|\cdot\|_{s, D}$ (see, e.g., [35]). For $D \subset \mathbb{R}^{3}, H(\operatorname{curl} ; D)$ and $H\left(\operatorname{div}_{\varepsilon} ; D\right)$ are the spaces of real or complex vector functions $\mathbf{u} \in L^{2}(D)^{3}$ with $\nabla \times \mathbf{u} \in L^{2}(D)^{3}$ and $\nabla \cdot(\varepsilon \mathbf{u}) \in L^{2}(D)$, respectively, endowed with the graph norms. Whenever $\varepsilon$ is the identity, we omit the subscript and simply write $H(\operatorname{div} ; D)$. We denote by $H_{0}^{1}(D), H_{0}(\operatorname{curl} ; D)$ and $H_{0}\left(\operatorname{div}_{\varepsilon} ; D\right)$ the subspaces of $H^{1}(D), H(\operatorname{curl} ; D)$ and $H\left(\operatorname{div}_{\varepsilon} ; D\right)$ of functions with zero trace, 
tangential trace, and normal trace, respectively, and by $H\left(\operatorname{curl}^{0} ; D\right)$ and $H\left(\operatorname{div}_{\varepsilon}^{0} ; D\right)$ the subspaces of $H(\operatorname{curl} ; D)$ and $H\left(\operatorname{div}_{\varepsilon} ; D\right)$ of curl-free and divergence-free functions, respectively. We also define $H_{0}\left(\operatorname{curl}, \operatorname{div}_{\varepsilon} ; D\right)=H_{0}(\operatorname{curl} ; D) \cap H\left(\operatorname{div}_{\varepsilon} ; D\right)$ and $H_{0}\left(\operatorname{curl}^{0}, \operatorname{div}_{\varepsilon}^{0} ; D\right)=H_{0}\left(\operatorname{curl}^{0} ; D\right) \cap H\left(\operatorname{div}_{\varepsilon}^{0} ; D\right)$. Finally, we denote by $\mathcal{H}(\partial D)$ the space of tangential traces of $H(\operatorname{curl} ; D)$ functions endowed with the norm $\|\cdot\|_{\mathcal{H}(\partial D)}$, and refer to [14 for its complete characterization in non-simply connected domains.

2.2. The low-frequency time-harmonic Maxwell equations in heterogeneous media. The physical problem we are interested in is the low-frequency timeharmonic Maxwell system (1.1)-(1.2), completed with Dirichlet boundary conditions on $\partial \Omega$ and flux conditions through the cavities of $\Omega_{0}$. Renaming the unknown field, the complete problem reads as follows. Find $\mathbf{u} \in H(\operatorname{curl} ; \Omega) \cap H\left(\operatorname{div}_{\varepsilon} ; \Omega_{0}\right)$ such that

$$
\begin{array}{rlrl}
\nabla \times\left(\mu^{-1} \nabla \times \mathbf{u}\right)+i \omega \sigma \mathbf{u}=\mathbf{J} & & \text { in } \Omega, \\
\nabla \cdot(\varepsilon \mathbf{u})=0 & & \text { in } \Omega_{0}, \\
\mathbf{n} \times \mathbf{u}=\mathbf{g} & & \text { on } \partial \Omega, \\
\left\langle\left.\varepsilon \mathbf{u}\right|_{\Omega_{0}} \cdot \mathbf{n}_{0, j}, 1\right\rangle_{\Gamma_{0, j}} & =0 & & \forall j=1, \ldots, J,
\end{array}
$$

where $\mathbf{n}$ is the outward normal unit vector to $\partial \Omega$, g is the tangential trace in $\mathcal{H}(\partial \Omega)$ of a function in $H(\operatorname{curl} ; \Omega), \mathbf{n}_{0, j}$ is the normal unit vector to $\Gamma_{0, j}$ pointing outside $\Omega_{0}$, and $\langle\cdot, \cdot\rangle_{\Gamma_{0, j}}$ denotes the duality product between $H^{-\frac{1}{2}}\left(\Gamma_{0, j}\right)$ and $H^{\frac{1}{2}}\left(\Gamma_{0, j}\right)$ with $L^{2}\left(\Gamma_{0, j}\right)$ as pivot space. We refer to [1] for a mathematical justification of this low-frequency model of time-harmonic Maxwell equations.

We consider the following regularized variational formulation of (2.1) $-(2.4)$. Find $\mathbf{u} \in H(\operatorname{curl} ; \Omega) \cap H\left(\operatorname{div}_{\varepsilon} ; \Omega_{0}\right)$ such that $\mathbf{n} \times \mathbf{u}=\mathbf{g}$ on $\partial \Omega,\left\langle\left.\varepsilon \mathbf{u}\right|_{\Omega_{0}} \cdot \mathbf{n}_{0, j}, 1\right\rangle_{\Gamma_{0, j}}=0$ for $j=1, \ldots, J$, and

$$
\begin{aligned}
& \int_{\Omega} \mu^{-1} \nabla \times \mathbf{u} \cdot \nabla \times \overline{\mathbf{v}} d \mathbf{x}+i \omega \int_{\Omega} \sigma \mathbf{u} \cdot \overline{\mathbf{v}} d \mathbf{x}+\int_{\Omega_{0}} \nu \nabla \cdot(\varepsilon \mathbf{u}) \nabla \cdot(\varepsilon \overline{\mathbf{v}}) d \mathbf{x} \\
& \quad=\int_{\Omega} \mathbf{J} \cdot \overline{\mathbf{v}} d \mathbf{x},
\end{aligned}
$$

for all $\mathbf{v} \in H_{0}(\operatorname{curl} ; \Omega) \cap H\left(\operatorname{div}_{\varepsilon} ; \Omega_{0}\right)$ with $\left\langle\left.\varepsilon \mathbf{v}\right|_{\Omega_{0}} \cdot \mathbf{n}_{0, j}, 1\right\rangle_{\Gamma_{0, j}}=0, j=1, \ldots, J$. Here, $\nu=\nu(\mathbf{x})$ is any positive bounded dimensional scalar function bounded away from zero that should be chosen in such a way that the magnitudes of the different terms at the left-hand side are balanced.

Although $\mathbf{J}$ is divergence free in $\Omega_{0}$, possible errors in the experimental recovering and/or numerical representation of $\mathbf{J}$ may give rise to source terms components that are not divergence free. In order to address this issue, we consider in the next section the strong problem corresponding to (2.5) with $\mathbf{J}$ replaced by a generic $\mathbf{F} \in L^{2}(\Omega)^{3}$.

2.3. The model problem. Let $\mathbf{F} \in L^{2}(\Omega)^{3}$. Then the function $\varepsilon^{-1} \mathbf{F} \in L^{2}(\Omega)^{3}$ admits the decomposition

$$
\varepsilon^{-1} \mathbf{F}=\mathbf{F}^{\prime}+\mathbf{F}^{\prime \prime}
$$

where $\mathbf{F}^{\prime} \in L^{2}(\Omega)^{3}$ is such that $\left.\nabla \cdot\left(\varepsilon \mathbf{F}^{\prime}\right)\right|_{\Omega_{0}}=0$ and $\left\langle\left.\varepsilon \mathbf{F}^{\prime}\right|_{\Omega_{0}} \cdot \mathbf{n}_{0, j}, 1\right\rangle_{\Gamma_{0, j}}=0$ for $j=1, \ldots, J$, while $\mathbf{F}^{\prime \prime}$ satisfies $\left.\mathbf{F}^{\prime \prime}\right|_{\Omega_{\sigma}}=\mathbf{0}$ and $\left.\mathbf{F}^{\prime \prime}\right|_{\Omega_{0}}=\nabla f$, with $f \in H^{1}\left(\Omega_{0}\right)$, $f=0$ on $\Gamma_{0,0}$ and $f$ constant, say $f=f_{j}$, on each $\Gamma_{0, j}$ for $j=1, \ldots, J$. This is a consequence of the decomposition (4.14) in [30], with $\Omega=\Omega_{0}, \Gamma_{\tau}=\partial \Omega_{0}$, $\Gamma_{\nu}=\emptyset$ and $\omega=\varepsilon$, and of Proposition 3.18 in [4, which can be easily generalized 
to the space $H_{0}\left(\operatorname{curl}^{0}, \operatorname{div}_{\varepsilon}^{0} ; \Omega_{0}\right)$. The decomposition in (2.6) is $\varepsilon$-orthogonal, i.e., orthogonal with respect to the weighted inner product $(\mathbf{v}, \mathbf{w})_{\varepsilon}=\int_{\Omega} \varepsilon \mathbf{v} \cdot \mathbf{w} d \mathbf{x}$.

We consider the following problem. Find $\mathbf{u} \in H(\operatorname{curl} ; \Omega) \cap H\left(\operatorname{div}_{\varepsilon} ; \Omega_{0}\right)$ such that

$$
\begin{aligned}
\nabla \times\left(\mu^{-1} \nabla \times \mathbf{u}\right)+i \omega \sigma \mathbf{u} & =\varepsilon \mathbf{F}^{\prime} & & \text { in } \Omega, \\
\nu \nabla \cdot(\varepsilon \mathbf{u}) & =-f & & \text { in } \Omega_{0}, \\
\mathbf{n} \times \mathbf{u} & =\mathbf{g} & & \text { on } \partial \Omega, \\
\lambda\left\langle\left.\varepsilon \mathbf{u}\right|_{\Omega_{0}} \cdot \mathbf{n}_{0, j}, 1\right\rangle_{\Gamma_{0, j}} & =f_{j} & & \forall j=1, \ldots, J,
\end{aligned}
$$

where $\lambda$ is any positive constant. Notice that for $\mathbf{F}=\mathbf{J}$, we have $\varepsilon \mathbf{F}^{\prime}=\mathbf{J}$ and $f=0$ in the decomposition (2.6), and problem (2.7)-(2.10) reduces to (2.1) $-(2.4)$.

We point out that in the LDG discretization of problem (2.7)-(2.10) we need to compute neither the elements $\mathbf{F}^{\prime}$ and $f$ in the decomposition of $\mathbf{F}$ nor the constants $f_{j}$. The only data that enter explicitly the formulation of the method are $\mathbf{F}$ and $\mathbf{g}$. This is due to the variational character of the method and the particular choice of the inhomogeneous flux conditions in (2.10) (see Remark 3.4 below).

We define the space $\mathbf{V}=H(\operatorname{curl} ; \Omega) \cap H\left(\operatorname{div}_{\varepsilon} ; \Omega_{0}\right)$, endowed with the norm

$$
\begin{aligned}
\|\mathbf{v}\|_{\mathbf{V}}^{2}=|\omega|\left\|\sigma_{\vartheta}^{\frac{1}{2}} \mathbf{v}\right\|_{0, \Omega}^{2}+\| \mu^{-\frac{1}{2}} \nabla & \times \mathbf{v}\left\|_{0, \Omega}^{2}+\right\| \nu^{\frac{1}{2}} \nabla \cdot(\varepsilon \mathbf{v}) \|_{0, \Omega_{0}}^{2} \\
& +\lambda \sum_{j=1}^{J}\left|\left\langle\left.\varepsilon \mathbf{v}\right|_{\Omega_{0}} \cdot \mathbf{n}_{0, j}, 1\right\rangle_{\Gamma_{0, j}}\right|^{2},
\end{aligned}
$$

with $\sigma_{\vartheta}=\sigma$ in $\Omega_{\sigma}$ and $\sigma_{\vartheta}=\vartheta I$ in $\Omega_{0}$, where $I$ is the identity and $\vartheta$ is a fixed positive dimensional constant.

The variational formulation corresponding to (2.7)-(2.10) is find $\mathbf{u} \in \mathbf{V}$ such that $\mathbf{n} \times \mathbf{u}=\mathbf{g}$ on $\partial \Omega, \lambda\left\langle\left.\varepsilon \mathbf{u}\right|_{\Omega_{0}} \cdot \mathbf{n}_{0, j}, 1\right\rangle_{\Gamma_{0, j}}=f_{j}$ for $j=1, \ldots, J$, and

$$
\begin{aligned}
\int_{\Omega} \mu^{-1} \nabla \times \mathbf{u} \cdot \nabla \times \overline{\mathbf{v}} d \mathbf{x}+i \omega \int_{\Omega} \sigma \mathbf{u} \cdot \overline{\mathbf{v}} d \mathbf{x}+\int_{\Omega_{0}} \nu \nabla \cdot(\varepsilon \mathbf{u}) \nabla \cdot(\varepsilon \overline{\mathbf{v}}) d \mathbf{x} \\
\quad=\int_{\Omega} \mathbf{F} \cdot \overline{\mathbf{v}} d \mathbf{x},
\end{aligned}
$$

for all $\mathbf{v} \in \mathbf{V}$, with $\mathbf{n} \times \mathbf{v}=\mathbf{0}$ on $\partial \Omega$ and $\left\langle\left.\varepsilon \mathbf{v}\right|_{\Omega_{0}} \cdot \mathbf{n}_{0, j}, 1\right\rangle_{\Gamma_{0, j}}=0, j=1, \ldots, J$.

Well-posedness of the above formulation is established in the following theorem.

Theorem 2.1. For any $\mathbf{F} \in L^{2}(\Omega)^{3}$ and $\mathbf{g} \in \mathcal{H}(\partial \Omega)$, the variational formulation (2.11) admits a unique solution and there exists a positive constant $C$ such that

$$
\|\mathbf{u}\|_{\mathbf{v}} \leq C\left(\|\mathbf{F}\|_{0, \Omega}+\|\mathbf{g}\|_{\mathcal{H}(\partial \Omega)}\right) .
$$

Moreover, $\mathbf{u}$ is solution to problem (2.7)-(2.10) if and only if $\mathbf{u}$ is solution to (2.11).

In the case where the domain is such that $H\left(\operatorname{curl} ; \Omega_{0}\right) \cap H_{0}\left(\operatorname{div} ; \Omega_{0}\right) \hookrightarrow H^{1}\left(\Omega_{0}\right)^{3}$ and the problem is driven by boundary conditions only, this result has been proved in [2] and [1]. The extension to our more general case is rather technical and will be given in detail in the appendix. One of the key ingredients necessary to prove Theorem 2.1 is to construct, under our assumptions on the domain, a continuous lifting of tangential traces with zero $\varepsilon$-divergence in $\Omega_{0}$ and zero flux conditions through $\Gamma_{0, j}, j=1 \ldots, J$. We do this in Proposition A.1, by using trace theorems recently proved in [15] and [16], and extended in [14] to domains with non-simply connected boundaries. 


\section{The LOCAL Discontinuous GALERKIN METHOD}

In this section, we formulate the LDG method for the discretization of problem (2.7) - (2.10). We assume from now on that

$$
\mathbf{g} \in L^{2}(\partial \Omega)^{3}
$$

3.1. Traces and discontinuous finite element spaces. We start by introducing certain trace operators and finite element spaces used in the definition of the method. Let $\mathcal{T}_{h}$ be a shape regular triangulation of the domain $\Omega$ into tetrahedra and/or parallelepipeds, with possible hanging nodes and aligned with the interfaces between different materials, so that $\mu, \mu^{-1}, \varepsilon$ and $\sigma$ are smooth within each element of $\mathcal{T}_{h}$. We set $\mathcal{T}_{h}^{0}:=\left.\mathcal{T}_{h}\right|_{\Omega_{0}}$ and have $\bar{\Omega}=\bigcup_{K \in \mathcal{T}_{h}} \bar{K}$ and $\bar{\Omega}_{0}=\bigcup_{K \in \mathcal{T}_{h}^{0}} \bar{K}$. We will denote by $h_{K}$ the diameter of the element $K \in \mathcal{T}_{h}$.

Faces. We define and characterize the faces of the triangulation $\mathcal{T}_{h}$. An interior face of $\mathcal{T}_{h}$ is defined as the (nonempty) two-dimensional interior of $\partial K^{+} \cap \partial K^{-}$, where $K^{+}$and $K^{-}$are two adjacent elements of $\mathcal{T}_{h}$, not necessarily matching. A boundary face of $\mathcal{T}_{h}$ is defined as the (nonempty) two-dimensional interior of $\partial K \cap \partial \Omega$, where $K$ is a boundary element of $\mathcal{T}_{h}$. We denote by $\mathcal{E}_{\mathcal{I}}$ the union of all interior faces of $\mathcal{T}_{h}$, by $\mathcal{E}_{\mathcal{D}}$ the union of all the boundary faces of $\mathcal{T}_{h}$, and by $\mathcal{E}=\mathcal{E}_{\mathcal{I}} \cup \mathcal{E}_{\mathcal{D}}$ the union of all faces of $\mathcal{T}_{h}$. Similarly, we denote by $\mathcal{E}^{0}$ the union of all faces of $\mathcal{T}_{h}^{0}$, and we write $\mathcal{E}_{\mathcal{I}}^{0}$ and $\mathcal{E}_{\partial}^{0}$ for the interior and boundary faces of $\mathcal{T}_{h}^{0}$.

Traces. Let $H^{s}\left(\mathcal{T}_{h}\right):=\left\{v:\left.v\right|_{K} \in H^{s}(K), K \in \mathcal{T}_{h}\right\}$ for $s>\frac{1}{2}$, endowed with the norm $\|v\|_{s, \mathcal{T}_{h}}^{2}=\sum_{K \in \mathcal{T}_{h}}\|v\|_{s, K}^{2}$. Then, the element-wise traces of functions in $H^{s}\left(\mathcal{T}_{h}\right)$ belong to $\operatorname{TR}(\mathcal{E}):=\Pi_{K \in \mathcal{T}_{h}} L^{2}(\partial K)$; they are double-valued on $\mathcal{E}_{\mathcal{I}}$ and single-valued on $\mathcal{E}_{\mathcal{D}}$. The space $L^{2}(\mathcal{E})$ can be identified with the functions in $\operatorname{TR}(\mathcal{E})$ for which the two trace values coincide. We define similarly $H^{s}\left(\mathcal{T}_{h}^{0}\right), \operatorname{TR}\left(\mathcal{E}^{0}\right)$, and $L^{2}\left(\mathcal{E}^{0}\right)$.

Trace operators. Let us introduce the following trace operators for piece-wise smooth functions. First, let $\mathbf{v} \in \operatorname{TR}(\mathcal{E})^{3}$ and $e \in \mathcal{E}$. If $e$ is an interior face in $\mathcal{E}_{\mathcal{I}}$, we denote by $K_{1}$ and $K_{2}$ the elements sharing $e$, by $\mathbf{n}_{i}$ the normal unit vector pointing exterior to $K_{i}$, and we set $\mathbf{v}_{i}=\left.\mathbf{v}\right|_{\partial K_{i}}, i=1,2$. We define the average and tangential jump of $\mathbf{v}$ at $\mathbf{x} \in e$ as

$\{\{\mathbf{v}\}\}=\left\{\begin{array}{ll}\frac{1}{2}\left(\mathbf{v}_{1}+\mathbf{v}_{2}\right) & \text { if } e \subset \mathcal{E}_{\mathcal{I}}, \\ \mathbf{v} & \text { if } e \subset \mathcal{E}_{\mathcal{D}},\end{array} \| \mathbf{v} \rrbracket_{T}= \begin{cases}\mathbf{n}_{1} \times \mathbf{v}_{1}+\mathbf{n}_{2} \times \mathbf{v}_{2} & \text { if } e \subset \mathcal{E}_{\mathcal{I}}, \\ \mathbf{n} \times \mathbf{v} & \text { if } e \subset \mathcal{E}_{\mathcal{D}},\end{cases}\right.$

and, if $e \subset \mathcal{E}_{\mathcal{I}}^{0}$, the normal jump of $\mathbf{v}$ at $\mathbf{x} \in e$ as

$$
\llbracket \mathbf{v} \rrbracket_{N}=\mathbf{v}_{1} \cdot \mathbf{n}_{1}+\mathbf{v}_{2} \cdot \mathbf{n}_{2} \quad \text { if } e \subset \mathcal{E}_{\mathcal{I}}^{0} .
$$

The normal jump of $\mathbf{v}$ will not be used on faces outside $\mathcal{T}_{h}^{0}$, and thus is left undefined. Similarly, we define for $\psi \in \mathrm{TR}\left(\mathcal{E}^{0}\right)$ the average and jump at $\mathbf{x} \in e$ as

$$
\left\{\{\psi\}=\left\{\begin{array}{ll}
\frac{1}{2}\left(\psi_{1}+\psi_{2}\right) & \text { if } e \subset \mathcal{E}_{\mathcal{I}}^{0}, \\
\psi & \text { if } e \subset \mathcal{E}_{\partial}^{0},
\end{array} \| \psi \rrbracket= \begin{cases}\psi_{1} \mathbf{n}_{1}+\psi_{2} \mathbf{n}_{2} & \text { if } e \subset \mathcal{E}_{\mathcal{I}}^{0}, \\
\psi \mathbf{n}_{0} & \text { if } e \subset \mathcal{E}_{\partial}^{0},\end{cases}\right.\right.
$$

where we recall that $\mathbf{n}_{0}$ denotes the outward normal unit to $\partial \Omega_{0}$. Note that the averages and jumps defined above are single-valued functions. 
If $\mathbf{v} \in H(\operatorname{curl} ; \Omega)$, then, for all $e \subset \mathcal{E}_{\mathcal{I}}$, the jump condition $\mathbf{n}_{1} \times \mathbf{v}_{1}+\mathbf{n}_{2} \times \mathbf{v}_{2}=\mathbf{0}$ holds true in $H_{00}^{-\frac{1}{2}}(e)^{3}$, and thus also in $L^{2}(e)^{3}$. For the definition of $H_{00}^{-\frac{1}{2}}(e)$, see, e.g., [35. Therefore $\llbracket \mathbf{v} \rrbracket_{T}$ is well defined and equal to zero on $\mathcal{E}_{\mathcal{I}}$. Similarly, for $\mathbf{v} \in H\left(\operatorname{div} ; \Omega_{0}\right)$, we have that $\llbracket \mathbf{v} \rrbracket_{N}$ is well defined and equal to zero on $\mathcal{E}_{\mathcal{I}}^{0}$. Furthermore, for the exact solution $\mathbf{u} \in \mathbf{V}$, owing to assumption (3.1), we have for a boundary face $e \subset \mathcal{E}_{\mathcal{D}}$ that $\llbracket \mathbf{u} \rrbracket_{T}=\mathbf{g}$ in $L^{2}(e)^{3}$, in addition to $\llbracket \mathbf{u} \rrbracket_{T}=\mathbf{0}$ on $\mathcal{E}_{I}$ and $\llbracket \varepsilon \mathbf{u} \rrbracket_{N}=0$ on $\mathcal{E}_{I}^{0}$.

Finite element spaces. Let $\underline{p}=\left\{p_{K}\right\}_{K \in \mathcal{T}_{h}}$ be a degree vector that assigns to each element $K \in \mathcal{T}_{h}$ a polynomial approximation order $p_{K} \geq 1$. The generic $h p$-finite element space of piece-wise polynomials is then given by

$$
S^{\underline{p}, 0}\left(\mathcal{T}_{h}\right):=\left\{u \in L^{2}(\Omega):\left.u\right|_{K} \in \mathcal{S}^{p_{K}}(K), \forall K \in \mathcal{T}_{h}\right\},
$$

where $\mathcal{S}^{p_{K}}(K)$ is the space $\mathcal{P}^{p_{K}}(K)$ of complex polynomials of degree at most $p_{K}$ in $K$ if $K$ is a tetrahedron, and the space $\mathcal{Q}^{p_{K}}(K)$ of complex polynomials of degree at most $p_{K}$ in each variable in $K$ if $K$ is a parallelepiped. The superscript 0 indicates that $S^{\underline{p}, 0}\left(\mathcal{T}_{h}\right) \subset L^{2}(\Omega)=H^{0}(\Omega)$. We define $S^{\underline{p}}, 0\left(\mathcal{T}_{h}^{0}\right)$ similarly.

3.2. Derivation of the LDG method. We introduce the auxiliary variables

$$
\begin{array}{lll}
\mathbf{s}=\mu^{-1} \mathbf{w} & \mathbf{w}=\nabla \times \mathbf{u} & \text { in } \Omega, \\
\varphi=\nu \rho & \rho=\nabla \cdot(\varepsilon \mathbf{u}) & \text { in } \Omega_{0} .
\end{array}
$$

Notice that $\mathbf{s} \in H(\operatorname{curl} ; \Omega), \mathbf{w} \in L^{2}(\Omega)^{3}, \varphi \in H^{1}\left(\Omega_{0}\right)$, and $\rho \in L^{2}\left(\Omega_{0}\right)$. By subtracting $\varepsilon$ times the gradient of equation (2.8) from equation (2.7), taking into account the above identities and that $\mathbf{F}=\varepsilon \mathbf{F}^{\prime}$ in $\Omega_{\sigma}$ and $\mathbf{F}=\varepsilon \mathbf{F}^{\prime}+\varepsilon \nabla f$ in $\Omega_{0}$, we obtain

$$
\begin{aligned}
\nabla \times \mathbf{s}+i \omega \sigma \mathbf{u}-\varepsilon \nabla \varphi & =\mathbf{F} & & \text { in } \Omega_{0} \\
\nabla \times \mathbf{s}+i \omega \sigma \mathbf{u} & =\mathbf{F} & & \text { in } \Omega_{\sigma}
\end{aligned}
$$

The LDG method is obtained by discretizing the first-order equations in (3.2)(3.4) in a discontinuous way. Notice that $\mathbf{s}$ is related to the magnetic field phasor given by $i \omega^{-1} \mu^{-1} \nabla \times \mathbf{u}$. In this context, however, $\mathbf{s}, \mathbf{w}, \varphi$, and $\rho$ are auxiliary variables introduced in order to derive the method and will be eliminated from the equations locally in an element-by-element manner. This local solvability gives the name to the LDG method. We refer to [17] and [18 for a discussion of this elimination process from a computational point of view.

Since the LDG method is defined element-wise, we fix $K \in \mathcal{T}_{h}$ and set $K_{0}=$ $K_{0}(K)=K$, if $K \subset \Omega_{0}$, and $K_{0}=K_{0}(K)=\emptyset$, if $K \subset \Omega_{\sigma}$. We proceed formally by multiplying in $K$ the first identities in (3.2) and (3.3) by test functions $\mathbf{z}$ and $\tau$, the second identities in (3.2) and (3.3) by test functions $\mathbf{t}$ and $\psi$, and equation (3.4) by a test function $\mathbf{v}$. By integration by parts and varying $K \in \mathcal{T}_{h}$, we obtain 
the following weak formulation:

$$
\begin{aligned}
& \int_{K} \mu^{-1} \mathbf{w} \cdot \overline{\mathbf{z}} d \mathbf{x}=\int_{K} \mathbf{s} \cdot \overline{\mathbf{z}} d \mathbf{x} \\
& \int_{K_{0}} \nu \rho \bar{\tau} d \mathbf{x}=\int_{K_{0}} \varphi \bar{\tau} d \mathbf{x} \\
& \int_{K} \mathbf{w} \cdot \overline{\mathbf{t}} d \mathbf{x}=\int_{K} \mathbf{u} \cdot \nabla \times \overline{\mathbf{t}} d \mathbf{x}-\int_{\partial K} \overline{\mathbf{t}} \cdot \mathbf{u} \times \mathbf{n}_{K} d s \\
& \int_{K_{0}} \rho \bar{\psi} d \mathbf{x}=-\int_{K_{0}} \varepsilon \mathbf{u} \cdot \nabla \bar{\psi} d \mathbf{x}+\int_{\partial K_{0}} \varepsilon \mathbf{u} \cdot\left(\bar{\psi} \mathbf{n}_{K_{0}}\right) d s \\
& \int_{K} \mathbf{s} \cdot \nabla \times \overline{\mathbf{v}} d \mathbf{x}-\int_{\partial K} \overline{\mathbf{v}} \cdot \mathbf{s} \times \mathbf{n}_{K} d s+i \omega \int_{K} \sigma \mathbf{u} \cdot \overline{\mathbf{v}} d \mathbf{x} \\
& \quad+\int_{K_{0}} \varphi \nabla \cdot(\varepsilon \overline{\mathbf{v}}) d \mathbf{x}-\int_{\partial K_{0}} \varphi(\varepsilon \overline{\mathbf{v}}) \cdot \mathbf{n}_{K_{0}} d s=\int_{K} \mathbf{F} \cdot \overline{\mathbf{v}} d \mathbf{x}
\end{aligned}
$$

for any $K \in \mathcal{T}_{h}$, where $\mathbf{n}_{K}$ is the outward normal unit vector to $\partial K$. The boundary integrals in (3.5) have to be understood as duality pairings.

We approximate $(\mathbf{w}, \rho, \mathbf{s}, \varphi, \mathbf{u})$ in (3.5) by functions $\left(\mathbf{w}_{h}, \rho_{h}, \mathbf{s}_{h}, \varphi_{h}, \mathbf{u}_{h}\right)$ in the finite element space $\mathbf{W}_{h} \times M_{h} \times \boldsymbol{\Sigma}_{h} \times Q_{h} \times \mathbf{V}_{h}$ chosen as

$$
\mathbf{W}_{h}=\boldsymbol{\Sigma}_{h}=\mathbf{V}_{h}=S \underline{\underline{p}}, 0\left(\mathcal{T}_{h}\right)^{3}, \quad M_{h}=Q_{h}=\left\{\left.\nabla_{h} \cdot\left(\varepsilon \mathbf{v}_{h}\right)\right|_{\Omega_{0}}: \mathbf{v}_{h} \in \mathbf{V}_{h}\right\}
$$

for a given degree distribution $p$ and with $\nabla_{h}$. denoting the element-wise divergence operator. This choice implies that $\nabla_{h} \times \mathbf{V}_{h} \subset \boldsymbol{\Sigma}_{h}=\mathbf{W}_{h}$, where $\nabla_{h} \times$ denotes the element-wise curl operator. Notice that the finite-dimensional spaces $M_{h}$ and $Q_{h}$ are not, in general, polynomial spaces. On the other hand, the unknowns belonging to these spaces are auxiliary and will be eliminated from the formulation.

The discrete version of (3.5) then reads as follows. Find $\left(\mathbf{w}_{h}, \rho_{h}, \mathbf{s}_{h}, \varphi_{h}, \mathbf{u}_{h}\right) \in$ $\mathbf{W}_{h} \times M_{h} \times \boldsymbol{\Sigma}_{h} \times Q_{h} \times \mathbf{V}_{h}$ such that, for any $K \in \mathcal{T}_{h}$ and for any choice of test functions $(\mathbf{z}, \tau, \mathbf{t}, \psi, \mathbf{v}) \in \mathbf{W}_{h} \times M_{h} \times \boldsymbol{\Sigma}_{h} \times Q_{h} \times \mathbf{V}_{h}$, we have

$$
\begin{aligned}
& \int_{K} \mu^{-1} \mathbf{w}_{h} \cdot \overline{\mathbf{z}} d \mathbf{x}=\int_{K} \mathbf{s}_{h} \cdot \overline{\mathbf{z}} d \mathbf{x} \\
& \int_{K_{0}} \nu \rho_{h} \bar{\tau} d \mathbf{x}=\int_{K_{0}} \varphi_{h} \bar{\tau} d \mathbf{x} \\
& \int_{K} \mathbf{w}_{h} \cdot \overline{\mathbf{t}} d \mathbf{x}=\int_{K} \mathbf{u}_{h} \cdot \nabla \times \overline{\mathbf{t}} d \mathbf{x}-\int_{\partial K} \overline{\mathbf{t}} \cdot \widehat{\mathbf{u}}_{h} \times \mathbf{n}_{K} d s, \\
& \int_{K_{0}} \rho_{h} \bar{\psi} d \mathbf{x}=-\int_{K_{0}} \varepsilon \mathbf{u}_{h} \cdot \nabla \bar{\psi} d \mathbf{x}+\int_{\partial K_{0}} \widehat{\widehat{\varepsilon \mathbf{u}}}_{h} \cdot\left(\bar{\psi} \mathbf{n}_{K_{0}}\right) d s, \\
& \int_{K} \mathbf{s}_{h} \cdot \nabla \times \overline{\mathbf{v}} d \mathbf{x}-\int_{\partial K} \overline{\mathbf{v}} \cdot \widehat{\mathbf{s}}_{h} \times \mathbf{n}_{K} d s+i \omega \int_{K} \sigma \mathbf{u}_{h} \cdot \overline{\mathbf{v}} d \mathbf{x} \\
& +\int_{K_{0}} \varphi_{h} \nabla \cdot(\varepsilon \overline{\mathbf{v}}) d \mathbf{x}-\int_{\partial K_{0}} \widehat{\widehat{\varphi}}_{h}(\varepsilon \overline{\mathbf{v}}) \cdot \mathbf{n}_{K_{0}} d s=\int_{K} \mathbf{F} \cdot \overline{\mathbf{v}} d \mathbf{x} .
\end{aligned}
$$

Here, $\widehat{\mathbf{u}}_{h}, \widehat{\mathbf{s}}_{h}, \widehat{\widehat{\varepsilon \mathbf{u}}}_{h}$ and $\widehat{\widehat{\varphi}}_{h}$ denote the so-called numerical fluxes which are approximations to the traces of $\mathbf{u}, \mathbf{s}, \varepsilon \mathbf{u}$ and $\varphi$ on $\partial K$. They are crucial for the stability as well as for the accuracy of the method and will be defined in the next section. The fluxes $\widehat{\mathbf{u}}_{h}$ and $\widehat{\mathbf{s}}_{h}$ are related to the curl-curl operator, whereas the fluxes $\widehat{\widehat{\varepsilon \mathbf{u}}}_{h}$ and $\widehat{\widehat{\varphi}}_{h}$ are associated with the grad-div operator in $\Omega_{0}$. 
Remark 3.1. If $\mu$ and $\nu$ are piece-wise constant, the auxiliary variables $\mathbf{w}$ and $\rho$ are not needed, and the method can be defined by introducing directly $\mathbf{s}=\mu^{-1} \nabla \times \mathbf{u}$ and $\varphi=\nu \nabla \cdot \mathbf{u}$.

3.3. The numerical fluxes. As in 6, we understand the numerical fluxes as follows. Given $\mathbf{u}$ and $\mathbf{s}$ in $H^{s}\left(\mathcal{T}_{h}\right)^{3}$ for $s>\frac{1}{2}$, the fluxes $\widehat{\mathbf{u}}=\widehat{\mathbf{u}}(\mathbf{u})$ and $\widehat{\mathbf{s}}=\widehat{\mathbf{s}}(\mathbf{s}, \mathbf{u})$ belong to $L^{2}(\mathcal{E})^{3}$. Similarly, for $\left.\mathbf{u}\right|_{\Omega_{0}} \in H^{s}\left(\mathcal{T}_{h}^{0}\right)^{3}$ and $\varphi \in H^{s}\left(\mathcal{T}_{h}^{0}\right), \widehat{\widehat{\varepsilon \mathbf{u}}}=\widehat{\widehat{\varepsilon \mathbf{u}}}\left(\left.\mathbf{u}\right|_{\Omega_{0}}, \varepsilon\right)$ belongs to $L^{2}\left(\mathcal{E}^{0}\right)^{3}$ and $\widehat{\hat{\varphi}}=\widehat{\varphi}\left(\varphi,\left.\mathbf{u}\right|_{\Omega_{0}}\right)$ to $L^{2}\left(\mathcal{E}^{0}\right)$. The fluxes are thus singlevalued on the union of faces. Furthermore, the fluxes $\widetilde{\mathbf{u}}$ and $\widehat{\widehat{\varepsilon \mathbf{u}}}$ are assumed to be independent of the auxiliary variables in order to be able to eliminate them from the system of equations.

We define the fluxes $\widehat{\mathbf{s}}$ and $\widehat{\mathbf{u}}$ face by face by adapting to the curl-curl operator the numerical fluxes considered in [19] and 24] for the Laplacian

$$
\begin{aligned}
& \widehat{\mathbf{s}}= \begin{cases}\{\mathbf{s}\}-\mathrm{a} \llbracket \mathbf{u} \rrbracket_{T}+\mathrm{b} \llbracket \mathbf{s} \rrbracket_{T} & \text { if } e \subset \mathcal{E}_{\mathcal{I}}, \\
\mathbf{s}-\mathrm{a}(\mathbf{n} \times \mathbf{u}-\mathbf{g}) & \text { if } e \subset \mathcal{E}_{\mathcal{D}},\end{cases} \\
& \widehat{\mathbf{u}}= \begin{cases}\{\mathbf{u}\}+\mathrm{b} \llbracket \mathbf{u} \rrbracket_{T} & \text { if } e \subset \mathcal{E}_{\mathcal{I}}, \\
\mathbf{g} \times \mathbf{n} & \text { if } e \subset \mathcal{E}_{\mathcal{D}} .\end{cases}
\end{aligned}
$$

We use a similar recipe for the grad-div fluxes and set

$$
\begin{gathered}
\widehat{\widehat{\varphi}}= \begin{cases}\{\varphi \varphi\}-\mathrm{c} \llbracket \varepsilon \mathbf{u} \rrbracket_{N}+\mathrm{d} \cdot \llbracket \varphi \rrbracket & \text { if } e \subset \mathcal{E}_{\mathcal{I}}^{0}, \\
-\lambda\left\langle\left.\varepsilon \mathbf{u}\right|_{\Omega_{0}} \cdot \mathbf{n}_{0, j} 1\right\rangle_{\Gamma_{0, j},}, & \text { if } e \subset \Gamma_{0, j} \quad \text { if } e \subset \Gamma_{0,0}, \\
0 & \text { if } e \subset \mathcal{E}_{\mathcal{I}}^{0},\end{cases} \\
\widehat{\widehat{\varepsilon} \mathbf{u}}= \begin{cases}\left\{\left\{\varepsilon \mathbf{u} \rrbracket-\mathrm{d} \llbracket \varepsilon \mathbf{u} \rrbracket_{N}\right.\right. & \text { if } e \subset \mathcal{E}_{\partial}^{0} . \\
\varepsilon \mathbf{u} & \end{cases}
\end{gathered}
$$

Here, a $\in L^{\infty}(\mathcal{E}), \mathrm{b} \in L^{\infty}\left(\mathcal{E}_{\mathcal{I}}\right), \mathrm{c} \in L^{\infty}\left(\mathcal{E}_{\mathcal{I}}^{0}\right)$, and $\mathrm{d} \in L^{\infty}\left(\mathcal{E}_{\mathcal{I}}^{0}\right)^{3}$ are real-valued functions still at our disposal. This completes the definition of the LDG method.

Let us make some comments about these fluxes.

- The fluxes introduced above are conservative in the sense of [6], and give rise to a consistent formulation (see Theorem 3.3 below).

- The parameters a and c are referred to as discontinuity stabilization parameters. They have to be positive and will be chosen depending on the local meshsize, polynomial degree, and on the coefficients $\mu$ and $\nu$. The parameters $\mathrm{b}$ and $\mathrm{d}$, on the other hand, are independent of $h$ and $p$; their purpose is to enhance the accuracy in the approximation of the auxiliary variables $\mathbf{s}$ and $\varphi$ that might be computed in a postprocessing step. Indeed, in [24] it has been shown for the Laplacian that a parameter like $b$ and $d$ can be selected in such a way that the auxiliary variable superconverges on Cartesian grids.

- The numerical flux $\widehat{\mathbf{u}}$ enforces the boundary condition (2.3) in a weak sense. Namely, for any $\mathbf{u} \in H^{s}\left(\mathcal{T}_{h}\right)^{3}$, we have that

$$
\mathbf{n} \times \widehat{\mathbf{u}}=\mathbf{g} \quad \text { on } \mathcal{E}_{\mathcal{D}},
$$

since $\mathbf{g}=\mathbf{n} \times(\mathbf{g} \times \mathbf{n})$. The flux $\widehat{\hat{\varphi}}$ imposes the condition $\varphi=0$ on $\Gamma_{0,0}$ and $\varphi=-\lambda\left\langle\left.\varepsilon \mathbf{u}_{h}\right|_{\Omega_{0}} \cdot \mathbf{n}_{0, j}, 1\right\rangle_{\Gamma_{0, j}}$ on $\Gamma_{0, j}, j=1, \ldots, J$. Since for the exact solution $\lambda\left\langle\left.\varepsilon \mathbf{u}\right|_{\Omega_{0}} \cdot \mathbf{n}_{0, j}, 1\right\rangle_{\Gamma_{0, j}}=f_{j}$ on $\Gamma_{0, j}$, the flux $\widehat{\hat{\varphi}}$ approximates the boundary condition 
$\varphi=-f_{j}$ on $\Gamma_{0, j}$. This is the reason why the constants $f_{j}$ do not appear explicitly in the formulation (see also Remark 3.4 below).

- Since the trace on $\Gamma_{0, j}$ of a function $\mathbf{v} \in H^{s}\left(\mathcal{T}_{h}^{0}\right)^{3}$ with $s>\frac{1}{2}$ actually belongs to $L^{2}\left(\Gamma_{0, j}\right)^{3}$, and $\varepsilon$ is smooth in each element, we have that $\left\langle\left.\varepsilon \mathbf{v}\right|_{\Omega_{0}} \cdot \mathbf{n}_{0, j}, 1\right\rangle_{\Gamma_{0, j}}=$ $\left.\int_{\Gamma_{0, j}} \varepsilon \mathbf{v}\right|_{\Omega_{0}} \cdot \mathbf{n}_{0, j} d s, j=1, \ldots, J$.

3.4. The mixed formulation of the LDG method. In this section, we cast the LDG method in a mixed form, as in [19], and prove existence and uniqueness of discrete solutions and consistency of the method. To do this, we sum the equations in (3.7) over all elements, and integrate back by parts. Then, by using the identities

$$
\begin{aligned}
\sum_{K \in \mathcal{T}_{h}} \int_{\partial K} \overline{\mathbf{t}} \cdot \mathbf{v} \times \mathbf{n}_{K} d s & =-\sum_{K \in \mathcal{T}_{h}} \int_{\partial K} \mathbf{v} \cdot \overline{\mathbf{t}} \times \mathbf{n}_{K} d s \\
& =-\int_{\mathcal{E}} \llbracket \mathbf{v} \rrbracket_{T} \cdot\left\{\{\overline{\mathbf{t}}\} d s+\int_{\mathcal{E}_{\mathcal{I}}}\{\mathbf{v}\}\right] \cdot \llbracket \overline{\mathbf{t}} \rrbracket_{T} d s \\
\sum_{K \in \mathcal{T}_{h}^{0}} \int_{\partial K} \mathbf{w} \cdot\left(\bar{\psi} \mathbf{n}_{K}\right) d s & =\int_{\mathcal{E}_{\mathcal{I}}^{0}}\left(\{\mathbf{w}\} \cdot \llbracket \bar{\psi} \rrbracket+\llbracket \mathbf{w} \rrbracket_{N}\{\{\bar{\psi}\}) d s+\int_{\mathcal{E}_{\partial}^{0}} \mathbf{w} \cdot\left(\bar{\psi} \mathbf{n}_{0}\right) d s\right.
\end{aligned}
$$

that hold true for all $\mathbf{v}, \mathbf{t} \in \operatorname{TR}(\mathcal{E})^{3}, \mathbf{w} \in \operatorname{TR}\left(\mathcal{E}^{0}\right)^{3}$, and $\psi \in \operatorname{TR}\left(\mathcal{E}^{0}\right)$, as well as the form of the numerical fluxes, we obtain the following formulation.

Mixed formulation. Find $\left(\mathbf{w}_{h}, \rho_{h}, \mathbf{s}_{h}, \varphi_{h}, \mathbf{u}_{h}\right) \in \mathbf{W}_{h} \times M_{h} \times \boldsymbol{\Sigma}_{h} \times Q_{h} \times \mathbf{V}_{h}$ such that

$$
\begin{aligned}
& \int_{\Omega} \mu^{-1} \mathbf{w}_{h} \cdot \overline{\mathbf{z}} d \mathbf{x}=\int_{\Omega} \mathbf{s}_{h} \cdot \overline{\mathbf{z}} d \mathbf{x}, \\
& \int_{\Omega_{0}} \nu \rho_{h} \bar{\tau} d \mathbf{x}=\int_{\Omega_{0}} \varphi_{h} \bar{\tau} d \mathbf{x}, \\
& \int_{\Omega} \mathbf{w}_{h} \cdot \overline{\mathbf{t}} d \mathbf{x}=\int_{\Omega} \nabla_{h} \times \mathbf{u}_{h} \cdot \overline{\mathbf{t}} d \mathbf{x}-\int_{\mathcal{E}_{\mathcal{I}}} \mathrm{b} \llbracket \mathbf{u}_{h} \rrbracket_{T} \cdot \llbracket \overline{\mathbf{t}} \rrbracket_{T} d s \\
& \quad-\int_{\mathcal{E}} \llbracket \mathbf{u}_{h} \rrbracket_{T} \cdot\{\{\overline{\mathbf{t}}\}\} d s+\int_{\mathcal{E}_{\mathcal{D}}} \mathbf{g} \cdot \overline{\mathbf{t}} d s, \\
& \int_{\Omega_{0}} \rho_{h} \bar{\psi} d \mathbf{x}=\int_{\Omega_{0}} \nabla_{h} \cdot\left(\varepsilon \mathbf{u}_{h}\right) \bar{\psi} d \mathbf{x}-\int_{\mathcal{E}_{I}^{0}} \mathrm{~d} \llbracket \varepsilon \mathbf{u}_{h} \rrbracket_{N} \cdot \llbracket \bar{\psi} \rrbracket d s-\int_{\mathcal{E}_{I}^{0}} \llbracket \varepsilon \mathbf{u}_{h} \rrbracket_{N}\{\{\bar{\psi}\}] d s, \\
& \int_{\Omega} \mathbf{s}_{h} \cdot \nabla_{h} \times \overline{\mathbf{v}} d \mathbf{x}-\int_{\mathcal{E}}\left\{\mathbf{s}_{h}\right\} \cdot \llbracket \overline{\mathbf{v}} \rrbracket_{T} d s-\int_{\mathcal{E}_{\mathcal{I}}} \mathrm{b} \llbracket \mathbf{s}_{h} \rrbracket_{T} \cdot \llbracket \overline{\mathbf{v}} \rrbracket_{T} d s+\int_{\mathcal{E}} \mathrm{a} \llbracket \mathbf{u}_{h} \rrbracket_{T} \cdot \llbracket \overline{\mathbf{v}} \rrbracket_{T} d s \\
& \quad+i \omega \int_{\Omega} \sigma \mathbf{u}_{h} \cdot \overline{\mathbf{v}} d \mathbf{x}+\int_{\Omega_{0}} \varphi_{h} \nabla_{h} \cdot(\varepsilon \overline{\mathbf{v}}) d \mathbf{x}-\int_{\mathcal{E}_{I}^{0}}\left\{\varphi_{h}\right\} \llbracket \varepsilon \overline{\mathbf{v}} \rrbracket_{N} d s \\
& \quad-\int_{\mathcal{E}_{\mathcal{I}}^{0}} \mathrm{~d} \cdot \llbracket \varphi_{h} \rrbracket \llbracket \varepsilon \overline{\mathbf{v}} \rrbracket_{N} d s+\int_{\mathcal{E}_{I}^{0}} \mathrm{c} \llbracket \varepsilon \mathbf{u}_{h} \rrbracket_{N} \llbracket \varepsilon \overline{\mathbf{v}} \rrbracket_{N} d s \\
& \quad+\lambda \sum_{j=1}^{J}\left\langle\left.\varepsilon \mathbf{u}_{h}\right|_{\Omega_{0}} \cdot \mathbf{n}_{0, j}, 1\right\rangle_{\Gamma_{0, j}}\left\langle\left.\varepsilon \overline{\mathbf{v}}\right|_{\Omega_{0}} \cdot \mathbf{n}_{0, j}, 1\right\rangle_{\Gamma_{0, j}}=\int_{\Omega} \mathbf{F} \cdot \overline{\mathbf{v}} d \mathbf{x}+\int_{\mathcal{E}_{\mathcal{D}}} \mathrm{ag} \cdot(\mathbf{n} \times \overline{\mathbf{v}}) d s,
\end{aligned}
$$

for all $(\mathbf{z}, \tau, \mathbf{t}, \psi, \mathbf{v}) \in \mathbf{W}_{h} \times M_{h} \times \boldsymbol{\Sigma}_{h} \times Q_{h} \times \mathbf{V}_{h}$. 
Remark 3.2. By using (3.9), the first two terms in the fifth equation of (3.10) can be expressed by

$$
\begin{aligned}
\int_{\Omega} \mathbf{s}_{h} \cdot \nabla_{h} & \times \overline{\mathbf{v}} d \mathbf{x}-\int_{\mathcal{E}}\left\{\left\{\mathbf{s}_{h}\right\} \cdot \llbracket \overline{\mathbf{v}} \rrbracket_{T} d s\right. \\
= & \left.\int_{\Omega} \mathbf{s}_{h} \cdot \nabla_{h} \times \overline{\mathbf{v}} d \mathbf{x}-\sum_{K \in \mathcal{T}_{h}} \int_{\partial K} \overline{\mathbf{v}} \cdot \mathbf{s}_{h} \times \mathbf{n}_{K} d s-\int_{\mathcal{E}_{\mathcal{I}}}\{\overline{\mathbf{v}}\}\right] \cdot \llbracket \mathbf{s}_{h} \rrbracket_{T} d s .
\end{aligned}
$$

The right-hand side is well defined for the exact solution $\mathbf{s}$ if we interpret the boundary integrals as duality pairings and take into account that $\llbracket \mathbf{s} \rrbracket_{T}=\mathbf{0}$ on $\mathcal{E}_{\mathcal{I}}$. Thus, for the exact solution $\mathbf{s}$ we understand the fifth equation in the above sense.

We prove existence and uniqueness of solutions and consistency of (3.10) in the following theorem. Notice that in order to have consistency we do not need any smoothness assumption on the exact solution in addition to (3.1).

Theorem 3.3. For strictly positive discontinuity stabilization parameters a and c, the LDG method defines a unique approximate solution $\left(\mathbf{w}_{h}, \rho_{h}, \mathbf{s}_{h}, \varphi_{h}, \mathbf{u}_{h}\right)$ in the space $\mathbf{W}_{h} \times M_{h} \times \boldsymbol{\Sigma}_{h} \times Q_{h} \times \mathbf{V}_{h}$. Furthermore, the LDG formulation (3.10) is consistent, i.e., the exact solution $(\mathbf{w}, \rho, \mathbf{s}, \varphi, \mathbf{u})$ satisfies (3.10), for all test functions $(\mathbf{z}, \tau, \mathbf{t}, \psi, \mathbf{v}) \in \mathbf{W}_{h} \times M_{h} \times \boldsymbol{\Sigma}_{h} \times Q_{h} \times \mathbf{V}_{h}$.

Proof. Since problem (3.10) is linear and finite dimensional, in order to prove existence and uniqueness of solutions, it is sufficient to prove that if $\mathbf{F}=\mathbf{0}$ and $\mathbf{g}=\mathbf{0}$, then $\mathbf{w}_{h}=\mathbf{s}_{h}=\mathbf{u}_{h}=\mathbf{0}$ and $\rho_{h}=\varphi_{h}=0$. Taking $(\mathbf{z}, \tau, \mathbf{t}, \psi, \mathbf{v})=$ $\left(\mathbf{w}_{h}, \rho_{h}, \mathbf{s}_{h}, \varphi_{h}, \mathbf{u}_{h}\right)$ in (3.10), subtracting the first and the second equations from the third and the fourth ones, respectively, and then subtracting the results from the fifth equation, we obtain

$$
\begin{aligned}
\int_{\Omega} \mu^{-1} \mathbf{w}_{h}^{2} d \mathbf{x} & +\int_{\mathcal{E}} \mathrm{a} \llbracket \mathbf{u}_{h} \rrbracket_{T}^{2} d s+i \omega \int_{\Omega} \sigma \mathbf{u}_{h}^{2} d \mathbf{x}+\int_{\Omega_{0}} \nu \rho_{h}^{2} d \mathbf{x} \\
& +\int_{\mathcal{E}_{I}^{0}} \mathrm{c} \llbracket \varepsilon \mathbf{u}_{h} \rrbracket_{N}^{2} d s+\lambda \sum_{j=1}^{J}\left\langle\left.\varepsilon \mathbf{u}_{h}\right|_{\Omega_{0}} \cdot \mathbf{n}_{0, j}, 1\right\rangle_{\Gamma_{0, j}}^{2}=0 .
\end{aligned}
$$

Taking into account that $\mu^{-1}$ is positive definite in $\Omega$ and $\nu$ is positive in $\Omega_{0}$, we have $\mathbf{w}_{h}=\mathbf{0}$ in $\Omega$ and $\rho_{h}=0$ in $\Omega_{0}$; since $\sigma$ is positive definite in $\Omega_{\sigma}$, then $\mathbf{u}_{h}=\mathbf{0}$ in $\Omega_{\sigma}$, and since a $>0, \mathrm{c}>0$ and $\lambda>0$, then $\llbracket \mathbf{u}_{h} \rrbracket_{T}=\mathbf{0}$ on $\mathcal{E}, \llbracket \varepsilon \mathbf{u}_{h} \rrbracket_{N}=0$ on $\mathcal{E}_{\mathcal{I}}^{0}$ and $\left\langle\left.\varepsilon \mathbf{u}_{h}\right|_{\Omega_{0}} \cdot \mathbf{n}_{0, j}, 1\right\rangle_{\Gamma_{0, j}}=0, j=1, \ldots, J$. Now, since $\boldsymbol{\Sigma}_{h}=\mathbf{W}_{h}$ and $Q_{h}=M_{h}$, taking $\mathbf{s}_{h}$ and $\varphi_{h}$ as test functions in the first and second equations of (3.10), respectively, from $\mathbf{w}_{h}=\mathbf{0}$ and $\rho_{h}=0$, we have $\mathbf{s}_{h}=\mathbf{0}$ in $\Omega$ and $\varphi_{h}=0$ in $\Omega_{0}$. Then, the third equation reduces to $\int_{\Omega} \nabla_{h} \times \mathbf{u}_{h} \cdot \overline{\mathbf{t}} d \mathbf{x}=0$, for all $\mathbf{t} \in \boldsymbol{\Sigma}_{h}$. Since $\nabla_{h} \times \mathbf{V}_{h} \subseteq \boldsymbol{\Sigma}_{h}$, we have $\nabla_{h} \times \mathbf{u}_{h}=\mathbf{0}$ in $\Omega$. Similarly, the fourth equation becomes $\int_{\Omega_{0}} \nabla_{h} \cdot\left(\varepsilon \mathbf{u}_{h}\right) \bar{\psi} d \mathbf{x}=0$, for all $\psi \in Q_{h}$. From the definition of $Q_{h}$, we can take $\psi=\nabla_{h} \cdot\left(\varepsilon \mathbf{u}_{h}\right)$ and obtain $\nabla_{h} \cdot\left(\varepsilon \mathbf{u}_{h}\right)=0$ in $\Omega_{0}$. From $\mathbf{u}_{h}=\mathbf{0}$ in $\Omega_{\sigma}$ and $\llbracket \mathbf{u}_{h} \rrbracket_{T}=\mathbf{0}$ on $\mathcal{E}$, we get $\mathbf{n}_{0} \times \mathbf{u}_{h}=\mathbf{0}$ on $\partial \Omega_{0}$. We can summarize the above conditions on $\mathbf{u}_{h}$ in $\Omega_{0}$ as $\left.\mathbf{u}_{h}\right|_{\Omega_{0}} \in H_{0}\left(\operatorname{curl}^{0}, \operatorname{div}_{\varepsilon}^{0} ; \Omega_{0}\right)$ and $\left\langle\left.\varepsilon \mathbf{u}_{h}\right|_{\Omega_{0}} \cdot \mathbf{n}_{0, j}, 1\right\rangle_{\Gamma_{0, j}}=0, j=1, \ldots, J$. This implies that $\mathbf{u}_{h}=\mathbf{0}$ also in $\Omega_{0}$ (see [30], formula (4.14) with $\Gamma_{\tau}=\partial \Omega_{0}, \Gamma_{\nu}=\emptyset$ and weight $\omega=\varepsilon$ ). This concludes the proof of the first part of the theorem.

Now let $(\mathbf{w}, \rho, \mathbf{s}, \varphi, \mathbf{u})$ be the exact solution. From $\mathbf{s}=\mu^{-1} \mathbf{w}$ and $\varphi=\nu \rho$, it is obvious that the first two equations are fulfilled, for any $\mathbf{z} \in \mathbf{W}_{h}$ and $\tau \in M_{h}$. Since $\llbracket \mathbf{u} \rrbracket_{T}=\mathbf{0}$ on $\mathcal{E}_{\mathcal{I}}$ and $\llbracket \mathbf{u} \rrbracket_{T}=\mathbf{g} \in L^{2}\left(\mathcal{E}_{\mathcal{D}}\right)^{3}$ on $\mathcal{E}_{\mathcal{D}}$, due to (3.1), taking into account 
that $\mathbf{w}=\nabla \times \mathbf{u}$, we have that the third equation is satisfied by $\mathbf{w}$ and $\mathbf{u}$, for all $\mathbf{t} \in \boldsymbol{\Sigma}_{h}$. Similarly, since $\llbracket \varepsilon \mathbf{u} \rrbracket_{N}=0$ on $\mathcal{E}_{\mathcal{I}}^{0}$, taking into account that $\varphi=\nabla \cdot(\varepsilon \mathbf{u})$, we have that the fourth equation is satisfied by $\varphi$ and $\mathbf{u}$, for all $\psi \in Q_{h}$. Finally, consider the fifth equation. Understanding the first two terms as in Remark 3.2 integrating by parts and observing that $\mathbf{u} \in \mathbf{V}, \mathbf{s} \in H(\operatorname{curl} ; \Omega)$ and $\varphi \in H^{1}\left(\Omega_{0}\right)$, together with the definition of $\{\{\varphi\}$, we get

$$
\begin{aligned}
\int_{\Omega} \nabla \times \mathbf{s} \cdot \overline{\mathbf{v}} d \mathbf{x} & +i \omega \int_{\Omega} \sigma \mathbf{u} \cdot \overline{\mathbf{v}} d \mathbf{x}-\int_{\Omega_{0}} \varepsilon \nabla \varphi \cdot \overline{\mathbf{v}} d \mathbf{x}+\int_{\mathcal{E}_{\partial}^{0}} \varphi(\varepsilon \overline{\mathbf{v}}) \cdot \mathbf{n} d s \\
& +\lambda \sum_{j=1}^{J}\left\langle\left.\varepsilon \mathbf{u}\right|_{\Omega_{0}} \cdot \mathbf{n}_{0, j}, 1\right\rangle_{\Gamma_{0, j}}\left\langle\left.\varepsilon \overline{\mathbf{v}}\right|_{\Omega_{0}} \cdot \mathbf{n}_{0, j}, 1\right\rangle_{\Gamma_{0, j}}=\int_{\Omega} \mathbf{F} \cdot \overline{\mathbf{v}} d \mathbf{x} .
\end{aligned}
$$

From (3.4) and the flux conditions (2.10), we obtain

$$
\int_{\mathcal{E}_{\partial}^{0}} \varphi(\varepsilon \overline{\mathbf{v}} \cdot \mathbf{n}) d s+\sum_{j=1}^{J} f_{j}\left\langle\left.\varepsilon \overline{\mathbf{v}}\right|_{\Omega_{0}} \cdot \mathbf{n}_{0, j}, 1\right\rangle_{\Gamma_{0, j}}=0,
$$

which is satisfied because $\left.\varphi\right|_{\partial \Omega_{0}}=\left.(\nu \nabla \cdot(\varepsilon \mathbf{u}))\right|_{\partial \Omega_{0}}=-\left.f\right|_{\partial \Omega_{0}}$, and $f$ is zero on $\Gamma_{0,0}$ and constant $f_{j}$ on $\Gamma_{0, j}, j=1, \ldots, J$. This completes the proof of the theorem.

Remark 3.4. The constants $f_{j}$ do not appear explicitly in the LDG formulation (3.10). As can be inferred from the proof of Theorem 3.3, this is due to the particular choice of the flux conditions in (2.10), whose purpose is, in fact, to cancel the terms containing the constants $f_{j}$, since they are not easily computable from the datum F. If we consider problem (2.7)-(2.10) with more general flux conditions

$$
\lambda\left\langle\left.\varepsilon \mathbf{u}\right|_{\Omega_{0}} \cdot \mathbf{n}_{0, j}, 1\right\rangle_{\Gamma_{0, j}}=\alpha_{j},
$$

for given constants $\alpha_{j}, j=1, \ldots, J$, the numerical flux $\widehat{\hat{\varphi}}$ on the faces belonging to $\Gamma_{0, j}, j=1, \ldots, J$, must be adjusted accordingly by setting

$$
\widehat{\hat{\varphi}}=\left(\alpha_{j}-f_{j}\right)-\lambda\left\langle\left.\varepsilon \mathbf{u}_{h}\right|_{\Omega_{0}} \cdot \mathbf{n}_{0, j}, 1\right\rangle_{\Gamma_{0, j}} .
$$

Consequently, the right-hand side in the last equation of (3.10) becomes

$$
\int_{\Omega} \mathbf{F} \cdot \overline{\mathbf{v}} d \mathbf{x}+\int_{\mathcal{E}_{\mathcal{D}}} \mathbf{a} \mathbf{g} \cdot(\mathbf{n} \times \overline{\mathbf{v}}) d s+\sum_{j=1}^{J}\left(\alpha_{j}-f_{j}\right)\left\langle\left.\varepsilon \overline{\mathbf{v}}\right|_{\Omega_{0}} \cdot \mathbf{n}_{0, j}, 1\right\rangle_{\Gamma_{0, j}} .
$$

3.5. The primal formulation of the LDG method. In this subsection, we eliminate the auxiliary variables $\mathbf{w}, \mathbf{s}, \rho$, and $\varphi$ from the mixed system in (3.10) and derive the primal formulation of the LDG method. This is possible since the fluxes $\widehat{\mathbf{u}}$ and $\widehat{\widehat{\varepsilon \mathbf{u}}}$ are chosen independently of $\mathbf{s}$ and $\varphi$.

Let us start by introducing the lifting operators $\mathcal{L}_{1}: L^{2}\left(\mathcal{E}_{\mathcal{I}}\right)^{3} \rightarrow \boldsymbol{\Sigma}_{h}, \mathcal{L}_{2}$ : $L^{2}(\mathcal{E})^{3} \rightarrow \boldsymbol{\Sigma}_{h}, \mathcal{M}_{1}: L^{2}\left(\mathcal{E}_{\mathcal{I}}^{0}\right)^{3} \rightarrow Q_{h}$, and $\mathcal{M}_{2}: L^{2}\left(\mathcal{E}_{\mathcal{I}}^{0}\right) \rightarrow Q_{h}$ defined by

$$
\begin{array}{ll}
\int_{\Omega} \mathcal{L}_{1}(\mathbf{v}) \cdot \overline{\mathbf{t}} d \mathbf{x}=\int_{\mathcal{E}_{\mathcal{I}}} \mathbf{v} \cdot \llbracket \overline{\mathbf{t}} \rrbracket_{T} d s, & \int_{\Omega} \mathcal{L}_{2}(\mathbf{v}) \cdot \overline{\mathbf{t}} d \mathbf{x}=\int_{\mathcal{E}} \mathbf{v} \cdot\{\overline{\mathbf{t}}\} d s \quad \forall \mathbf{t} \in \mathbf{\Sigma}_{h}, \\
\int_{\Omega_{0}} \mathcal{M}_{1}(\mathbf{v}) \bar{\psi} d \mathbf{x}=\int_{\mathcal{E}_{I}^{0}} \mathbf{v} \cdot \llbracket \bar{\psi} \rrbracket d s, & \int_{\Omega_{0}} \mathcal{M}_{2}(v) \bar{\psi} d \mathbf{x}=\int_{\mathcal{E}_{\bar{I}}^{0}} v\left\{\{\bar{\psi}\} d s \quad \forall \psi \in Q_{h},\right.
\end{array}
$$

as well as the lifting $\mathbf{G}_{\mathcal{D}} \in \boldsymbol{\Sigma}_{h}$ of the boundary datum given by

$$
\int_{\Omega} \mathbf{G}_{\mathcal{D}} \cdot \overline{\mathbf{t}} d \mathbf{x}=\int_{\mathcal{E}_{\mathcal{D}}} \mathbf{g} \cdot \overline{\mathbf{t}} d \mathbf{x} \quad \forall \mathbf{t} \in \mathbf{\Sigma}_{h} .
$$


Denoting by $\Pi_{\boldsymbol{\Sigma}_{h}}$ and $\Pi_{Q_{h}}$ the $L^{2}$-projections onto $\mathbf{W}_{h}=\boldsymbol{\Sigma}_{h}$ and $M_{h}=Q_{h}$, the first and second equation in (3.10) can be written as $\mathbf{s}_{h}=\boldsymbol{\Pi}_{\boldsymbol{\Sigma}_{h}}\left(\mu^{-1} \mathbf{w}_{h}\right)$ and $\varphi_{h}=\Pi_{Q_{h}}\left(\nu \rho_{h}\right)$. Then, from the third and fourth equations in (3.10), we obtain

$$
\begin{aligned}
\mathbf{s}_{h} & =\Pi_{\boldsymbol{\Sigma}_{h}}\left[\mu^{-1}\left(\nabla_{h} \times \mathbf{u}_{h}-\mathcal{L}\left(\llbracket \mathbf{u}_{h} \rrbracket_{T}\right)+\mathbf{G}_{\mathcal{D}}\right)\right] \\
\varphi_{h} & =\Pi_{Q_{h}}\left[\nu\left(\nabla_{h} \cdot\left(\varepsilon \mathbf{u}_{h}\right)-\mathcal{M}\left(\llbracket \varepsilon \mathbf{u}_{h} \rrbracket_{N}\right)\right)\right]
\end{aligned}
$$

with the compact notation $\mathcal{L}\left(\llbracket \mathbf{u}_{h} \rrbracket_{T}\right):=\mathcal{L}_{1}\left(\mathrm{~b} \llbracket \mathbf{u}_{h} \rrbracket_{T}\right)+\mathcal{L}_{2}\left(\llbracket \mathbf{u}_{h} \rrbracket_{T}\right)$, with $\mathrm{b} \llbracket \mathbf{u}_{h} \rrbracket_{T}$ understood as being restricted to $\mathcal{E}_{\mathcal{I}}$, and $\mathcal{M}\left(\llbracket \varepsilon \mathbf{u}_{h} \rrbracket_{N}\right):=\mathcal{M}_{1}\left(\mathrm{~d} \llbracket \varepsilon \mathbf{u}_{h} \rrbracket_{N}\right)+\mathcal{M}_{2}\left(\llbracket \varepsilon \mathbf{u}_{h} \rrbracket_{N}\right)$. Since $\nabla_{h} \times \mathbf{V}_{h} \subseteq \boldsymbol{\Sigma}_{h}$ and $\left.\nabla_{h} \cdot\left(\varepsilon \mathbf{V}_{h}\right)\right|_{\Omega_{0}}=Q_{h}$, identities (3.11) and (3.12) can be used in the fifth equation of (3.10), giving rise to the so-called primal formulation of the LDG discretization of (2.7) $-(2.10)$, in the variable $\mathbf{u}$ only.

Primal formulation. Find $\mathbf{u}_{h} \in \mathbf{V}_{h}$ such that, for all $\mathbf{v} \in \mathbf{V}_{h}$,

$$
\mathcal{B}_{h}\left(\mathbf{u}_{h}, \mathbf{v}\right):=\mathcal{A}_{h}\left(\mathbf{u}_{h}, \mathbf{v}\right)+\mathcal{I}_{h}\left(\mathbf{u}_{h}, \mathbf{v}\right)+i \omega \int_{\Omega} \sigma \mathbf{u}_{h} \cdot \overline{\mathbf{v}} d \mathbf{x}+\mathcal{J}\left(\mathbf{u}_{h}, \mathbf{v}\right)=\mathcal{F}_{h}(\mathbf{v}),
$$

where the forms $\mathcal{A}_{h}, \mathcal{I}_{h}$ (interior penalty form) and $\mathcal{J}$ are defined by

$$
\begin{aligned}
\mathcal{A}_{h}(\mathbf{u}, \mathbf{v})= & \int_{\Omega} \mu^{-1}\left(\nabla_{h} \times \mathbf{u}-\mathcal{L}\left(\llbracket \mathbf{u} \rrbracket_{T}\right)\right) \cdot\left(\nabla_{h} \times \overline{\mathbf{v}}-\mathcal{L}\left(\llbracket \overline{\mathbf{v}} \rrbracket_{T}\right)\right) d \mathbf{x} \\
& \quad+\int_{\Omega_{0}} \nu\left(\nabla_{h} \cdot(\varepsilon \mathbf{u})-\mathcal{M}\left(\llbracket \varepsilon \mathbf{u} \rrbracket_{N}\right)\right)\left(\nabla_{h} \cdot(\varepsilon \overline{\mathbf{v}})-\mathcal{M}\left(\llbracket \varepsilon \overline{\mathbf{v}} \rrbracket_{N}\right)\right) d \mathbf{x}, \\
\mathcal{I}_{h}(\mathbf{u}, \mathbf{v})= & \int_{\mathcal{E}} \mathrm{a} \llbracket \mathbf{u} \rrbracket_{T} \cdot \llbracket \overline{\mathbf{v}} \rrbracket_{T} d s+\int_{\mathcal{E}_{\mathcal{I}}^{0}} \mathrm{c} \llbracket \varepsilon \mathbf{u} \rrbracket_{N} \llbracket \varepsilon \overline{\mathbf{v}} \rrbracket_{N} d s, \\
\mathcal{J}(\mathbf{u}, \mathbf{v})= & \lambda \sum_{j=1}^{J}\left\langle\left.\varepsilon \mathbf{u}\right|_{\Omega_{0}} \cdot \mathbf{n}_{0, j}, 1\right\rangle_{\Gamma_{0, j}}\left\langle\left.\varepsilon \overline{\mathbf{v}}\right|_{\Omega_{0}} \cdot \mathbf{n}_{0, j}, 1\right\rangle_{\Gamma_{0, j}},
\end{aligned}
$$

and the linear form $\mathcal{F}_{h}$ by

$$
\mathcal{F}_{h}(\mathbf{v})=\int_{\Omega} \mathbf{F} \cdot \overline{\mathbf{v}} d \mathbf{x}-\int_{\Omega} \mu^{-1} \mathbf{G}_{\mathcal{D}} \cdot\left(\nabla_{h} \times \overline{\mathbf{v}}-\mathcal{L}\left(\llbracket \overline{\mathbf{v}} \rrbracket_{T}\right)\right) d \mathbf{x}+\int_{\mathcal{E}_{\mathcal{D}}} \mathrm{a} \mathbf{g} \cdot(\mathbf{n} \times \overline{\mathbf{v}}) d s
$$

For discrete test and trial functions, the primal form (3.13) of the LDG method, together with (3.11) and (3.12), is equivalent to the mixed system (3.10). However, unlike (3.10), the formulation (3.13) is no longer consistent, due to the discrete nature of the lifting operators. Nevertheless, the form $\mathcal{B}_{h}(\cdot, \cdot)$ has the continuity and coercivity properties that allow us to carry out an error analysis in a straightforward way by using Strang's lemma. Regarding this point, our approach differs from the analysis in [6].

Remark 3.5. Other DG methods can be defined by modifying the definitions of $\mathcal{A}_{h}(\mathbf{u}, \mathbf{v}), \mathcal{I}_{h}(\mathbf{u}, \mathbf{v})$, and $\mathcal{F}_{h}(\mathbf{v})$ in (3.13). The interior penalty (IP) method and its nonsymmetric variant (NIP), for instance, can be obtained by taking in (3.13) the 
same $\mathcal{I}_{h}(\mathbf{u}, \mathbf{v})$ as in the LDG method, and instead of $\mathcal{A}_{h}(\mathbf{u}, \mathbf{v})$ and $\mathcal{F}_{h}(\mathbf{v})$,

$$
\begin{aligned}
\mathcal{A}_{h}^{\mathrm{IP}}(\mathbf{u}, \mathbf{v})= & \int_{\Omega} \mu^{-1} \nabla_{h} \times \mathbf{u} \cdot \nabla_{h} \times \overline{\mathbf{v}} d \mathbf{x}-\int_{\mathcal{E}} \llbracket \mathbf{u} \rrbracket_{T} \cdot\left\{\left\{\mu^{-1} \nabla_{h} \times \overline{\mathbf{v}}\right\}\right\} d s \\
& -\int_{\mathcal{E}} \llbracket \overline{\mathbf{v}} \rrbracket_{T} \cdot\left\{\left\{\mu^{-1} \nabla_{h} \times \mathbf{u}\right\} d s+\int_{\Omega_{0}} \nu \nabla_{h} \cdot(\varepsilon \mathbf{u}) \nabla_{h} \cdot(\varepsilon \overline{\mathbf{v}}) d \mathbf{x}\right. \\
& -\int_{\mathcal{E}_{\mathcal{I}}^{0}} \llbracket \varepsilon \mathbf{u} \rrbracket_{N}\left\{\left\{\nu \nabla_{h} \cdot(\varepsilon \overline{\mathbf{v}})\right\}\right\} d s-\int_{\mathcal{E}_{\mathcal{I}}^{0}} \llbracket \varepsilon \overline{\mathbf{v}} \rrbracket_{N}\left\{\left\{\nu \nabla_{h} \cdot(\varepsilon \mathbf{u})\right\} d s,\right. \\
\mathcal{F}_{h}^{\mathrm{IP}}(\mathbf{v})= & \int_{\Omega} \mathbf{F} \cdot \overline{\mathbf{v}} d \mathbf{x}-\int_{\mathcal{E}_{\mathcal{D}}} \mathbf{g} \cdot \mu^{-1} \nabla_{h} \times \overline{\mathbf{v}} d s+\int_{\mathcal{E}_{\mathcal{D}}} \mathrm{a} \mathbf{g} \cdot(\mathbf{n} \times \overline{\mathbf{v}}) d s
\end{aligned}
$$

and

$$
\begin{aligned}
& \mathcal{A}_{h}^{\mathrm{NIP}}(\mathbf{u}, \mathbf{v})= \int_{\Omega} \mu^{-1} \nabla_{h} \times \mathbf{u} \cdot \nabla_{h} \times \overline{\mathbf{v}} d \mathbf{x}+\int_{\mathcal{E}} \llbracket \mathbf{u} \rrbracket_{T} \cdot\left\{\left\{\mu^{-1} \nabla_{h} \times \overline{\mathbf{v}}\right\}\right\} d s \\
&\left.-\int_{\mathcal{E}} \llbracket \overline{\mathbf{v}} \rrbracket_{T} \cdot\left\{\mu^{-1} \nabla_{h} \times \mathbf{u}\right\}\right\} d s+\int_{\Omega_{0}} \nu \nabla_{h} \cdot(\varepsilon \mathbf{u}) \nabla_{h} \cdot(\varepsilon \overline{\mathbf{v}}) d \mathbf{x} \\
&+\int_{\mathcal{E}_{\mathcal{I}}^{0}} \llbracket \varepsilon \mathbf{u} \rrbracket_{N}\left\{\left\{\nu \nabla_{h} \cdot(\varepsilon \overline{\mathbf{v}})\right\} d s-\int_{\mathcal{E}_{\mathcal{I}}^{0}} \llbracket \varepsilon \overline{\mathbf{v}} \rrbracket_{N}\left\{\left\{\nu \nabla_{h} \cdot(\varepsilon \mathbf{u})\right\} d s\right.\right. \\
& \mathcal{F}_{h}^{\mathrm{NIP}}(\mathbf{v})=\int_{\Omega} \mathbf{F} \cdot \overline{\mathbf{v}} d \mathbf{x}+\int_{\mathcal{E}_{\mathcal{D}}} \mathbf{g} \cdot \mu^{-1} \nabla_{h} \times \overline{\mathbf{v}} d s+\int_{\mathcal{E}_{\mathcal{D}}} \mathbf{a} \mathbf{g} \cdot(\mathbf{n} \times \overline{\mathbf{v}}) d s
\end{aligned}
$$

These formulations can also be derived by using the same mixed formulation as for the LDG method, and defining appropriately the numerical fluxes (see [6]). The analysis of the IP and NIP methods can be carried out in an almost identical manner to the one of the LDG method presented in the next section. A slight difference consists in a restriction on the choice of the stabilization parameters a and $\mathrm{c}$ in the IP method which in fact have to be large enough. We refer to [6] and [18] for an extensive discussion and comparison of different DG methods for diffusion problems, from a theoretical and a computational point of view.

\section{ERROR ANALYSIS}

The aim of this section is to present an $h p$-error analysis of the LDG method introduced in Section 3, based on its primal formulation (3.13). Although we use the same setting of [6], our analysis differs from the one presented there since we directly work on the discrete form (3.13), taking into account nonconsistency terms by Strang's lemma. This approach in the analysis of DG methods seems to be new and more suited for $h p$-version approaches.

Our analysis is carried out under the assumption that the coefficients of the electric permittivity tensor are piece-wise constant; i.e.,

$$
\varepsilon_{i j} \in \mathcal{S}^{0,0}\left(\mathcal{T}_{h}\right), \quad i, j=1,2,3
$$

so that the spaces $Q_{h}$ and $M_{h}$ consist of piece-wise polynomials. 
The main result (see Theorem 4.11 below) consists in error estimates, in a suitable energy-norm, of the form

$$
\begin{aligned}
\left\|\mathbf{u}-\mathbf{u}_{h}\right\|_{h}^{2} \leq C & \sum_{K \in \mathcal{T}_{h}} \frac{h_{K}^{2 \min \left(p_{K}, s_{K}\right)}}{p_{K}^{2 s_{K}-1}}\left(\|\mathbf{u}\|_{s_{K}+1, K}^{2}+\left\|\mu^{-1} \nabla \times \mathbf{u}\right\|_{s_{K}, K}^{2}\right) \\
& +C \sum_{K \in \mathcal{T}_{h}^{0}} \frac{h_{K}^{2 \min \left(p_{K}, s_{K}\right)}}{p_{K}^{2 s_{K}-1}}\|\nu \nabla \cdot(\varepsilon \mathbf{u})\|_{s_{K}, K}^{2},
\end{aligned}
$$

for exact solutions $\mathbf{u}$ that satisfy $\mathbf{u} \in H^{s_{K}+1}(K)^{3}, \mu^{-1} \nabla \times \mathbf{u} \in H^{s_{K}}(K)^{3}$, for all $K \in$ $\mathcal{T}_{h}$, and $\nu \nabla \cdot(\varepsilon \mathbf{u}) \in H^{s_{K}}(K)$, for all $K \in \mathcal{T}_{h}^{0}$, with local regularity exponents $s_{K} \geq 1$. These estimates are optimal in the local meshsizes $h_{K}$ and slightly suboptimal in the local approximation degree $p_{K}$. Furthermore, in Theorem 4.11 we also make explicit the dependence on the local material properties.

The outline of this section is as follows. In subsection 4.1 we define the discontinuity stabilization parameters a and $\mathrm{c}$ in terms of the local meshsize, approximation degree and magnetic permeability. Subection 4.2 is devoted to establish $h p$-stability estimates for the lifting operators $\mathcal{L}$ and $\mathcal{M}$ in the definition of the primal formulation. These estimates will be crucial in subsection 4.3 where we prove continuity and coercivity properties of the bilinear form $\mathcal{B}_{h}(\cdot, \cdot)$. Based on Strang's lemma, we derive our main $h p$-error estimates in subsection 4.4 In subsection 4.5 we recover error estimates for the auxiliary variables $\mathbf{s}$ and $\varphi$ used in the derivation of the LDG method. Recall that the variable $\mathbf{s}$ is related to the magnetic field, and therefore its computation might be of interest. We conclude in subsection 4.6 by investigating the stability of the discrete problem with respect to the data.

4.1. The discontinuity stabilization parameters. In this subsection, we define the discontinuity stabilization parameters a and c in terms of the "local meshsize", "local polynomial degree" and "local magnetic permeability". This allows us to obtain continuity and coercivity constants independent of global bounds for these quantities.

Let us start by introducing the functions $\mathrm{h}$ and $\mathrm{p}$ in $L^{\infty}(\mathcal{E})$, related to the local meshsize and polynomial degree, defined as

$$
\begin{aligned}
& \mathrm{h}=\mathrm{h}(\mathbf{x}):= \begin{cases}\min \left\{h_{K}, h_{K^{\prime}}\right\} & \text { if } \mathbf{x} \text { is in the interior of } \partial K \cap \partial K^{\prime}, \\
h_{K} & \text { if } \mathbf{x} \text { is in the interior of } \partial K \cap \partial \Omega,\end{cases} \\
& \mathrm{p}=\mathrm{p}(\mathbf{x}):= \begin{cases}\max \left\{p_{K}, p_{K^{\prime}}\right\} & \text { if } \mathbf{x} \text { is in the interior of } \partial K \cap \partial K^{\prime}, \\
p_{K} & \text { if } \mathbf{x} \text { is in the interior of } \partial K \cap \partial \Omega .\end{cases}
\end{aligned}
$$

Regarding the magnetic permeability, we assume $\mu$ to be Lipschitz continuous in $K$, for any $K \in \mathcal{T}_{h}$. This implies that $\left.\mu\right|_{K}$ can be extended up to $\partial K$, and we denote this extension by $\mu_{K}$. Therefore, for any $K \in \mathcal{T}_{h}$, there are positive constants $m_{K}$ and $M_{K}$ such that

$$
m_{K} \leq \lambda_{i}\left(\mu_{K}(\mathbf{x})\right) \leq M_{K} \quad \forall \mathbf{x} \in \bar{K},
$$

where $\lambda_{i}\left(\mu_{K}(\mathbf{x})\right), i=1,2,3$, are the eigenvalues of $\mu_{K}(\mathbf{x})$. Note that, for any $K \in \mathcal{T}_{h}$, the constants $m_{K}$ and $M_{K}$ satisfy $0<m \leq m_{K}$ and $M_{K} \leq M<+\infty$, where $m$ is the uniform ellipticity constant of $\mu$ and $M$ is the reciprocal of the uniform ellipticity constant of $\mu^{-1}$. 
We choose the scalar function $\nu$ in the formulation of the problem as $\nu(\mathbf{x})=$ $1 /|\mu(\mathbf{x})|$, for all $\mathbf{x} \in \Omega_{0}$, where $|\mu(\mathbf{x})|$ is the spectral norm of the tensor $\mu(\mathbf{x})(|\mu(\mathbf{x})|$ simply reduces to $\mu(\mathbf{x})$ whenever $\mu$ is a scalar function). Then we also have that $\nu$ satisfies

$$
\frac{1}{M_{K}} \leq \nu_{K}(\mathbf{x}) \leq \frac{1}{m_{K}} \quad \forall \mathbf{x} \in \bar{K}
$$

for any $K \in \mathcal{T}_{h}^{0}$, where we have defined $\nu_{K}$ in the same way as $\mu_{K}$.

We make the additional assumption that there exists $\kappa>0$ such that

$$
\frac{M_{K}}{m_{K}} \leq \kappa \quad \forall K \in \mathcal{T}_{h}, \forall \mathcal{T}_{h} .
$$

Whenever $\mu$ is a piece-wise constant scalar function, (4.3) holds true with $\kappa=1$. For $\mu$ piece-wise constant tensor, $\kappa$ in (4.3) expresses the maximum anisotropy among the different materials. We set

$\mathrm{m}=\mathrm{m}(\mathbf{x}):= \begin{cases}\min \left\{\left|\mu_{K}(\mathbf{x})\right|,\left|\mu_{K^{\prime}}(\mathbf{x})\right|\right\} & \text { if } \mathbf{x} \text { is in the interior of } \partial K \cap \partial K^{\prime}, \\ \left|\mu_{K}(\mathbf{x})\right| & \text { if } \mathbf{x} \text { is in the interior of } \partial K \cap \partial \Omega .\end{cases}$

We are now ready to define the discontinuity stabilization parameters a and $c$ in terms of $\mathrm{h}, \mathrm{p}$ and $\mathrm{m}$. They are chosen as

$$
\mathrm{a}=\alpha \mathrm{h}^{-1} \mathrm{p}^{2} \mathrm{~m}^{-1} \quad \text { in } L^{\infty}(\mathcal{E}), \quad \mathrm{c}=\alpha \mathrm{h}^{-1} \mathrm{p}^{2} \mathrm{~m}^{-1} \quad \text { in } L^{\infty}\left(\mathcal{E}_{\mathcal{I}}^{0}\right),
$$

with $\alpha>0$ independent of the meshsize, approximation order, and the magnetic permeability. The parameters $\mathrm{b}$ and $\mathrm{d}$ are taken to be of order one; i.e.,

$$
\|\mathrm{b}\|_{L^{\infty}\left(\mathcal{E}_{\mathcal{I}}\right)} \leq \delta, \quad\|\mathrm{d}\|_{L^{\infty}\left(\mathcal{E}_{\mathcal{I}}^{0}\right)^{3}} \leq \delta,
$$

with $\delta \geq 0$ independent of $h$ and $p$.

Remark 4.1. The choice of the stabilization parameters of order $p^{2} / h$ is the $h p$ extension of the choice in 6] for $h$-version DG methods for the Laplacian. This choice balances the interior penalty terms in $\mathcal{I}_{h}(\cdot, \cdot)$ with the stability estimates in Proposition 4.2 below for the lifting operators $\mathcal{L}$ and $\mathcal{M}$, or, equivalently, with the inverse estimate (4.6) below.

Stabilization parameters of order $p^{2} / h$ can also be found in the $h p$-literature on DG methods for diffusion problems (see, e.g., 34], 41] and [43, where different error analyses are developed). The choice $p / h$ is investigated in [34] for the NIP method, still leading to a suboptimal error bound in $p$. Furthermore, an improved $p$-bound has been recently obtained in 32 for two-dimensional reaction-diffusion problems on affine quadrilateral grids with hanging nodes, for solutions belonging to certain "augmented" Sobolev spaces. The same result can be established in our case, leading to $h p$-optimal bounds on structured grids, provided that the corresponding approximation properties can be extended to three space-dimensions.

4.2. The lifting operators. In this section, we derive $h p$-stability estimates for the lifting operators introduced in subsection 3.5. To do this, we define the space

$$
\mathbf{V}(h):=\left\{\mathbf{v}=\mathbf{w}_{h}+\mathbf{w} \mid \mathbf{w}_{h} \in \mathbf{V}_{h}, \mathbf{w} \in \mathbf{V} \text { with } \mathbf{n} \times \mathbf{w} \in L^{2}(\partial \Omega)^{3}\right\} .
$$

Owing to (3.1), the exact solution $\mathbf{u}$ belongs to $\mathbf{V}(h)$. 
Proposition 4.2. Let $\mathcal{L}$ and $\mathcal{M}$ be the lifting operators defined in subsection 3.5. Under the above assumptions on $\mu, \nu$ and $\varepsilon$, and assumption (4.5) on the parameters $\mathrm{b}$ and $\mathrm{d}$, we have that, for all $\mathbf{v} \in \mathbf{V}(h)$,

$$
\begin{aligned}
& \left\|\mu^{-\frac{1}{2}} \mathcal{L}\left(\llbracket \mathbf{v} \rrbracket_{T}\right)\right\|_{0, \Omega} \leq C_{\text {lift }} \kappa(\delta+1)\left\|\mathrm{h}^{-\frac{1}{2}} \mathrm{pm}^{-\frac{1}{2}} \llbracket \mathbf{v} \rrbracket_{T}\right\|_{0, \mathcal{E}}, \\
& \left\|\nu^{\frac{1}{2}} \mathcal{M}\left(\llbracket \varepsilon \mathbf{v} \rrbracket_{N}\right)\right\|_{0, \Omega_{0}} \leq C_{\text {lift }} \kappa(\delta+1)\left\|\mathrm{h}^{-\frac{1}{2}} \mathrm{pm}^{-\frac{1}{2}} \llbracket \varepsilon \mathbf{v} \rrbracket_{N}\right\|_{0, \mathcal{E}_{I}^{0}},
\end{aligned}
$$

with a constant $C_{\text {lift }}>0$ only depending on the shape regularity of the mesh. Moreover, for $\mathbf{G}_{\mathcal{D}}$ defined in subsection 3.5, we have

$$
\left\|\mu^{-\frac{1}{2}} \mathbf{G}_{\mathcal{D}}\right\|_{0, \Omega} \leq C_{\text {lift }} \kappa\left\|\mathrm{h}^{-\frac{1}{2}} \mathrm{pm}^{-\frac{1}{2}} \mathbf{g}\right\|_{0, \mathcal{E}_{\mathcal{D}}} .
$$

Proof. Let us first recall the inverse inequality

$$
\|q\|_{0, \partial K}^{2} \leq C_{\mathrm{inv}} \frac{p_{K}^{2}}{h_{K}}\|q\|_{0, K}^{2} \quad \forall q \in \mathcal{S}^{p_{K}}(K),
$$

with a constant $C_{\text {inv }}>0$ only depending on the shape regularity of the mesh. For two-dimensional elements, the proof of (4.6) can be found in [44, formula (4.6.4) of Theorem 4.76]; for three-space dimensions, the proof is analogous (see also [34]).

From the definition of $\mathcal{L}$ and $\mathcal{M}$ in terms of $\mathcal{L}_{i}$ and $\mathcal{M}_{i}, i=1,2$ (see subsection (3.5), the bounds for $\mathcal{L}$ and $\mathcal{M}$ can be proved by combining estimates for $\mathcal{L}_{i}$ and $\mathcal{M}_{i}, i=1,2$. We develop in detail the proof of the following estimate for $\mathcal{L}_{1}$ :

$$
\left\|\mu^{-\frac{1}{2}} \mathcal{L}_{1}\left(\mathrm{~b} \llbracket \mathbf{v} \rrbracket_{T}\right)\right\|_{0, \Omega} \leq C_{\text {lift }} \kappa \delta\left\|\mathrm{h}^{-\frac{1}{2}} \mathrm{pm}^{-\frac{1}{2}} \llbracket \mathbf{v} \rrbracket_{T}\right\|_{0, \mathcal{E}_{\mathcal{I}}} .
$$

Recall that, for $\mathbf{v}=\mathbf{w}_{h}+\mathbf{w} \in \mathbf{V}(h)$, we have $\llbracket \mathbf{v} \rrbracket_{T}=\llbracket \mathbf{w}_{h} \rrbracket_{T}$ on $\mathcal{E}_{\mathcal{I}}$. Denoting by $\boldsymbol{\Pi}_{\boldsymbol{\Sigma}_{h}}$ the $L^{2}$-projection onto $\boldsymbol{\Sigma}_{h}$, by the definition of the operator $\mathcal{L}_{1}$ and the Cauchy-Schwarz inequality, we have

$$
\begin{aligned}
\left\|\mu^{-\frac{1}{2}} \mathcal{L}_{1}\left(\mathrm{~b} \llbracket \mathbf{v} \rrbracket_{T}\right)\right\|_{0, \Omega} & =\sup _{\mathbf{z} \in L^{2}(\Omega)^{3}} \frac{\int_{\Omega} \mathcal{L}_{1}\left(\mathrm{~b} \llbracket \mathbf{v} \rrbracket_{T}\right) \cdot \mu^{-\frac{1}{2}} \overline{\mathbf{z}} d \mathbf{x}}{\|\mathbf{z}\|_{0, \Omega}} \\
& =\sup _{\mathbf{z} \in L^{2}(\Omega)^{3}} \frac{\int_{\Omega} \mathcal{L}_{1}\left(\mathrm{~b} \llbracket \mathbf{v} \rrbracket_{T}\right) \cdot \Pi_{\boldsymbol{\Sigma}_{h}}\left(\mu^{-\frac{1}{2}} \overline{\mathbf{z}}\right) d \mathbf{x}}{\|\mathbf{z}\|_{0, \Omega}} \\
& =\sup _{\mathbf{z} \in L^{2}(\Omega)^{3}} \frac{\int_{\mathcal{E}_{\mathcal{I}}} \mathbf{b} \llbracket \mathbf{v} \rrbracket_{T} \cdot \llbracket \Pi_{\boldsymbol{\Sigma}_{h}}\left(\mu^{-\frac{1}{2}} \overline{\mathbf{z}}\right) \rrbracket_{T} d s}{\|\mathbf{z}\|_{0, \Omega}} \\
& \leq \delta \sup _{\mathbf{z} \in L^{2}(\Omega)^{3}} \frac{\left\|\mathrm{h}^{-\frac{1}{2}} \mathrm{pm}^{-\frac{1}{2}} \llbracket \mathbf{v} \rrbracket_{T}\right\|_{0, \mathcal{E}_{\mathcal{I}}}\left\|\mathrm{h}^{\frac{1}{2}} \mathbf{p}^{-1} \mathrm{~m}^{\frac{1}{2}} \llbracket \boldsymbol{\Pi}_{\boldsymbol{\Sigma}_{h}}\left(\mu^{-\frac{1}{2}} \mathbf{z}\right) \rrbracket_{T}\right\|_{0, \mathcal{E}_{\mathcal{I}}}}{\|\mathbf{z}\|_{0, \Omega}} .
\end{aligned}
$$

Then, by using conditions (4.2) on $\mu$, the definitions of $\llbracket \cdot \rrbracket_{T}, \mathrm{~m}, \mathrm{~h}$ and $\mathrm{p}$, the inverse inequality (4.6), and properties of the $L^{2}$-projection, we obtain

$$
\begin{aligned}
\left\|\mathrm{h}^{\frac{1}{2}} \mathrm{p}^{-1} \mathrm{~m}^{\frac{1}{2}} \llbracket \boldsymbol{\Pi}_{\boldsymbol{\Sigma}_{h}}\left(\mu^{-\frac{1}{2}} \mathbf{z}\right) \rrbracket_{T}\right\|_{0, \mathcal{E}_{\mathcal{I}}}^{2} \leq 2 \sum_{K \in \mathcal{T}_{h}} \frac{h_{K} M_{K}}{p_{K}^{2}}\left\|\mathbf{n}_{K} \times \boldsymbol{\Pi}_{\boldsymbol{\Sigma}_{h}}\left(\mu^{-\frac{1}{2}} \mathbf{z}\right)\right\|_{0, \partial K}^{2} \\
\leq 2 C_{\mathrm{inv}} \sum_{K \in \mathcal{T}_{h}} M_{K}\left\|\boldsymbol{\Pi}_{\boldsymbol{\Sigma}_{h}}\left(\mu^{-\frac{1}{2}} \mathbf{z}\right)\right\|_{0, K}^{2} \leq 2 C_{\mathrm{inv}} \sum_{K \in \mathcal{T}_{h}} M_{K}\left\|\mu^{-\frac{1}{2}} \mathbf{z}\right\|_{0, K}^{2} \\
\leq 2 C_{\mathrm{inv}} \sum_{K \in \mathcal{T}_{h}} \frac{M_{K}}{m_{K}}\|\mathbf{z}\|_{0, K}^{2} \leq 2 C_{\mathrm{inv}} \kappa\|\mathbf{z}\|_{0, \Omega}^{2},
\end{aligned}
$$

where in the last step we used (4.3). This proves the desired estimate for $\mathcal{L}_{1}$ in (4.7). Analogous estimates can be obtained for $\mathcal{M}_{1}$ and $\mathcal{M}_{2}$, since assumption (4.1) on $\varepsilon$ guarantees that $Q_{h}$ is a polynomial space, as well as for $\mathcal{L}_{2}$, recalling 
that $\llbracket \mathbf{v} \rrbracket_{T} \in L^{2}\left(\mathcal{E}_{\mathcal{D}}\right)^{3}$ on $\mathcal{E}_{\mathcal{D}}$. Then the bounds for $\mathcal{L}$ and $\mathcal{M}$ immediately follow. Since $\mathbf{G}_{\mathcal{D}}=\mathcal{L}\left(\llbracket \mathbf{u} \rrbracket_{T}\right)$, the same arguments give the bound for $\mathbf{G}_{\mathcal{D}}$.

4.3. Continuity, coercivity, and error bound. In this section, we establish continuity and coercivity properties of the form $\mathcal{B}_{h}$. To do this, we introduce the seminorm $|\cdot|_{h}$ given by

$$
\begin{aligned}
|\mathbf{v}|_{h}^{2}=\left\|\mu^{-\frac{1}{2}} \nabla_{h} \times \mathbf{v}\right\|_{0, \Omega}^{2}+\left\|\mathrm{h}^{-\frac{1}{2}} \mathrm{pm}^{-\frac{1}{2}} \llbracket \mathbf{v} \rrbracket_{T}\right\|_{0, \mathcal{E}}^{2} \\
+\left\|\nu^{\frac{1}{2}} \nabla_{h} \cdot(\varepsilon \mathbf{v})\right\|_{0, \Omega_{0}}^{2}+\left\|\mathrm{h}^{-\frac{1}{2}} \mathrm{pm}^{-\frac{1}{2}} \llbracket \varepsilon \mathbf{v} \rrbracket_{N}\right\|_{0, \mathcal{E}_{\mathcal{I}}^{0}}^{2},
\end{aligned}
$$

as well as the norm $\|\cdot\|_{h}$

$$
\|\mathbf{v}\|_{h}^{2}=|\mathbf{v}|_{h}^{2}+|\omega|\left\|\sigma^{\frac{1}{2}} \mathbf{v}\right\|_{0, \Omega}^{2}+\lambda \sum_{j=1}^{J}\left|\left\langle\left.\varepsilon \mathbf{v}\right|_{\Omega_{0}} \cdot \mathbf{n}_{0, j}, 1\right\rangle_{\Gamma_{0, j}}\right|^{2} .
$$

That (4.9) is actually a norm in $\mathbf{V}(h)$ is proved in the following proposition.

Proposition 4.3. The quantity defined in (4.9) is a norm in $\mathbf{V}(h)$.

Proof. From $\|\mathbf{v}\|_{h}=0$, we immediately have $\mathbf{v}=\mathbf{0}$ in $\Omega_{\sigma}, \llbracket \mathbf{v} \rrbracket_{T}=0$ on $\mathcal{E}$ and $\llbracket \varepsilon \mathbf{v} \rrbracket_{N}=0$ on $\mathcal{E}_{\mathcal{I}}^{0}$; i.e., $\mathbf{v} \in H_{0}(\operatorname{curl} ; \Omega) \cap H\left(\operatorname{div}_{\varepsilon} ; \Omega_{0}\right)$. Now, from $\mathbf{v}=\mathbf{0}$ in $\Omega_{\sigma}$ and $\mathbf{v} \in H_{0}(\operatorname{curl} ; \Omega)$, it follows that $\mathbf{n}_{0} \times\left.\mathbf{v}\right|_{\Omega_{\sigma}}=\mathbf{0}$ on the interface $\Gamma=\partial \Omega_{\sigma} \cap \partial \Omega_{0}$, and therefore $\mathbf{n}_{0} \times\left.\mathbf{v}\right|_{\Omega_{0}}=\mathbf{0}$ on $\partial \Omega_{0}$. From $\nabla \times \mathbf{v}=\mathbf{0}$ in $\Omega, \mathbf{n}_{0} \times \mathbf{v}=\mathbf{0}$ on $\partial \Omega_{0}$, $\nabla \cdot(\varepsilon \mathbf{v})=0$ in $\Omega_{0}$ and $\left\langle\left.\varepsilon \mathbf{v}\right|_{\Omega_{0}} \cdot \mathbf{n}_{0, j}, 1\right\rangle_{\Gamma_{0, j}}=0, j=1 \ldots, J$, we get $\mathbf{v}=\mathbf{0}$ also in $\Omega_{0}$ (see, e.g., formula (4.14) in [30] with $\Omega=\Omega_{0}, \Gamma_{\tau}=\partial \Omega_{0}, \Gamma_{\nu}=\emptyset$, and $\omega=\varepsilon$ ), which concludes the proof.

Let us first prove continuity and coercivity properties for the LDG forms in (3.13).

Lemma 4.4. Assume the above hypotheses on $\mu, \nu, \varepsilon$ and on the coefficients in the definition of the numerical fluxes. Then the following continuity property holds true:

$$
\left|\mathcal{A}_{h}(\mathbf{w}, \mathbf{v})+\mathcal{I}_{h}(\mathbf{w}, \mathbf{v})\right| \leq C|\mathbf{w}|_{h}|\mathbf{v}|_{h} \quad \forall \mathbf{w}, \mathbf{v} \in \mathbf{V}(h)
$$

with a constant $C$ only depending on $\alpha, \delta, \kappa$, and $C_{\text {lift }}$.

Proof. For $\mathbf{w}, \mathbf{v} \in \mathbf{V}(h)$, we have

$$
\begin{aligned}
&\left|\mathcal{A}_{h}(\mathbf{w}, \mathbf{v})+\mathcal{I}_{h}(\mathbf{w}, \mathbf{v})\right| \\
& \leq\left\|\mu^{-\frac{1}{2}}\left[\nabla_{h} \times \mathbf{w}-\mathcal{L}\left(\llbracket \mathbf{w} \rrbracket_{T}\right)\right]\right\|_{0, \Omega}\left\|\mu^{-\frac{1}{2}}\left[\nabla_{h} \times \mathbf{v}-\mathcal{L}\left(\llbracket \mathbf{v} \rrbracket_{T}\right)\right]\right\|_{0, \Omega} \\
&+\left\|\nu^{\frac{1}{2}}\left[\nabla_{h} \cdot(\varepsilon \mathbf{w})-\mathcal{M}\left(\llbracket \varepsilon \mathbf{w} \rrbracket_{N}\right)\right]\right\|_{0, \Omega_{0}}\left\|\nu^{\frac{1}{2}}\left[\nabla_{h} \cdot(\varepsilon \mathbf{v})-\mathcal{M}\left(\llbracket \varepsilon \mathbf{v} \rrbracket_{N}\right)\right]\right\|_{0, \Omega_{0}} \\
&+\alpha\left\|\mathrm{h}^{-\frac{1}{2}} \mathrm{pm}^{-\frac{1}{2}} \llbracket \mathbf{w} \rrbracket_{T}\right\|_{0, \mathcal{E}}\left\|\mathrm{h}^{-\frac{1}{2}} \mathrm{pm}^{-\frac{1}{2}} \llbracket \mathbf{v} \rrbracket_{T}\right\|_{0, \mathcal{E}} \\
&+\alpha\left\|\mathrm{h}^{-\frac{1}{2}} \mathrm{pm}^{-\frac{1}{2}} \llbracket \varepsilon \mathbf{w} \rrbracket_{N}\right\|_{0, \mathcal{E}_{\mathcal{I}}^{0}}\left\|\mathrm{~h}^{-\frac{1}{2}} \mathrm{pm}^{-\frac{1}{2}} \llbracket \varepsilon \mathbf{v} \rrbracket_{N}\right\|_{0, \mathcal{E}_{\mathcal{I}}^{0}}
\end{aligned}
$$

From Proposition 4.2, we have $\left\|\mathcal{L}\left(\llbracket \mathbf{z} \rrbracket_{T}\right)\right\|_{0, \Omega} \leq C|\mathbf{z}|_{h}$ and $\left\|\mathcal{M}\left(\llbracket \varepsilon \mathbf{z} \rrbracket_{N}\right)\right\|_{0, \Omega_{0}} \leq C|\mathbf{z}|_{h}$, for $\mathbf{z}=\mathbf{w}$ and $\mathbf{z}=\mathbf{v}$, and the result immediately follows.

Lemma 4.5. Assume the above hypotheses on $\mu, \nu, \varepsilon$ and on the coefficients in the definition of the numerical fluxes. The coercivity property

$$
\mathcal{A}_{h}(\mathbf{v}, \mathbf{v})+\mathcal{I}_{h}(\mathbf{v}, \mathbf{v}) \geq C|\mathbf{v}|_{h}^{2} \quad \forall \mathbf{v} \in \mathbf{V}_{h}
$$

holds true for any choice of $\alpha>0$. The constant $C$ depends on $\alpha, \delta, \kappa$ and $C_{\text {lift }}$. 
Proof. We have

$$
\begin{aligned}
\mathcal{A}_{h}(\mathbf{v}, \mathbf{v})+\mathcal{I}_{h}(\mathbf{v}, \mathbf{v})= & \int_{\Omega}\left\{\mu^{-1}\left[\nabla_{h} \times \mathbf{v}-\mathcal{L}\left(\llbracket \mathbf{v} \rrbracket_{T}\right)\right]\right\}^{2} d \mathbf{x} \\
& +\int_{\Omega_{0}}\left\{\nu\left[\nabla_{h} \cdot(\varepsilon \mathbf{v})-\mathcal{M}\left(\llbracket \varepsilon \mathbf{v} \rrbracket_{N}\right)\right]\right\}^{2} d \mathbf{x} \\
& +\alpha\left\|\mathrm{h}^{-\frac{1}{2}} \mathrm{pm}^{-\frac{1}{2}} \llbracket \mathbf{v} \rrbracket_{T}\right\|_{0, \mathcal{E}}^{2}+\alpha\left\|\mathrm{h}^{-\frac{1}{2}} \mathrm{pm}^{-\frac{1}{2}} \llbracket \varepsilon \mathbf{v} \rrbracket_{N}\right\|_{0, \mathcal{E}_{I}^{0}}^{2} .
\end{aligned}
$$

The first term at the right-hand side can be bounded by

$$
\begin{aligned}
\left\|\mu^{-\frac{1}{2}} \nabla_{h} \times \mathbf{v}\right\|_{0, \Omega}^{2} & -2 \int_{\Omega} \mu^{-1} \nabla_{h} \times \mathbf{v} \cdot\left[\mathcal{L}\left(\llbracket \mathbf{v} \rrbracket_{T}\right)\right] d \mathbf{x}+\left\|\mu^{-\frac{1}{2}}\left[\mathcal{L}\left(\llbracket \mathbf{v} \rrbracket_{T}\right)\right]\right\|_{0, \Omega}^{2} \\
\geq & (1-\chi)\left\|\mu^{-\frac{1}{2}} \nabla_{h} \times \mathbf{v}\right\|_{0, \Omega}^{2}+\left(1-\frac{1}{\chi}\right)\left\|\mu^{-\frac{1}{2}}\left[\mathcal{L}\left(\llbracket \mathbf{v} \rrbracket_{T}\right)\right]\right\|_{0, \Omega}^{2},
\end{aligned}
$$

with $\chi>0$ still at our disposal. Similarly, the second term at the right-hand side can be bounded by

$$
(1-\chi)\left\|\nu^{\frac{1}{2}} \nabla_{h} \cdot(\varepsilon \mathbf{v})\right\|_{0, \Omega_{0}}^{2}+\left(1-\frac{1}{\chi}\right)\left\|\nu^{\frac{1}{2}}\left[\mathcal{M}\left(\llbracket \varepsilon \mathbf{v} \rrbracket_{N}\right)\right]\right\|_{0, \Omega_{0}}^{2} .
$$

Therefore, using the estimates of Proposition 4.2 and taking the $\chi$ that satisfies the inequalities

$$
\frac{C_{\text {lift }}^{2} \kappa^{2}(\delta+1)^{2}}{C_{\text {lift }}^{2} \kappa^{2}(\delta+1)^{2}+\alpha}<\chi<1
$$

we obtain the result.

Proposition 4.6. Assume the above hypotheses on $\mu, \nu, \varepsilon$ and on the coefficients in the definition of the numerical fluxes. The following continuity and coercivity properties hold true:

$$
\begin{array}{rlrl}
\left|\mathcal{B}_{h}(\mathbf{w}, \mathbf{v})\right| & \leq C_{\text {cont }}\|\mathbf{w}\|_{h}\|\mathbf{v}\|_{h} & & \forall \mathbf{w}, \mathbf{v} \in \mathbf{V}(h), \\
\left|\mathcal{B}_{h}(\mathbf{v}, \mathbf{v})\right| \geq C_{\text {coer }}\|\mathbf{v}\|_{h}^{2} & & \forall \mathbf{v} \in \mathbf{V}_{h},
\end{array}
$$

with $C_{\text {cont }}$ and $C_{\text {coer }}$ only depending on $\alpha, \delta, \kappa$ and $C_{\text {lift. }}$.

Proof. Since $\left|\mathcal{B}_{h}(\mathbf{v}, \mathbf{v})\right|=\left[\left(\mathcal{A}_{h}(\mathbf{v}, \mathbf{v})+\mathcal{I}_{h}(\mathbf{v}, \mathbf{v})+\mathcal{J}(\mathbf{v}, \mathbf{v})\right)^{2}+\omega^{2}\left\|\sigma^{\frac{1}{2}} \mathbf{v}\right\|_{0, \Omega}^{4}\right]^{\frac{1}{2}}$, the continuity and coercivity properties follow from Lemmas 4.4 and 4.5 and the definition of the norm $\|\cdot\|_{h}$.

As already pointed out, the primal formulation (3.13) that our analysis is based on is not consistent due to the discrete nature of the lifting operators. However, from Proposition 4.6 and Strang's lemma (see, e.g., 22, Theorem 4.2.2]), we immediately have the following error bound.

Theorem 4.7. Assume the above hypotheses on $\mu, \nu, \varepsilon$ and on the coefficients in the definition of the numerical fluxes. Then we have

$$
\left\|\mathbf{u}-\mathbf{u}_{h}\right\|_{h} \leq\left(1+\frac{C_{\text {cont }}}{C_{\text {coer }}}\right) \inf _{\mathbf{v} \in \mathbf{V}_{h}}\|\mathbf{u}-\mathbf{v}\|_{h}+\frac{1}{C_{\text {coer }}} \sup _{\mathbf{w} \in \mathbf{V}_{h}} \frac{\left|\mathcal{B}_{h}(\mathbf{u}, \mathbf{w})-\mathcal{F}_{h}(\mathbf{w})\right|}{\|\mathbf{w}\|_{h}} .
$$

Remark 4.8. In order to analyze stability properties of discrete solutions with respect to the data, the continuity of the functional $\mathcal{F}_{h}(\cdot)$ with respect to the norm $\|\cdot\|_{h}$ has to be investigated. This is not straightforward since $\|\cdot\|_{h}$ does not contain the $L^{2}$-norm over $\Omega_{0}$. In subsection [4.6, we prove a discrete Poincaré inequality 
that allows us to address this issue in the particular case where $\mu$ is the identity. In the general case of discontinuous permeabilities, we obtain the same stability estimates provided that the datum $\mathbf{F}$ satisfies certain restrictions. Also this point is addressed in subsection 4.6

4.4. $h p$-error estimates. In this section, we estimate the terms at the right-hand side in the error bound established in Theorem 4.7 and derive a priori error estimates for piece-wise smooth solutions. In order to do that, we need the following $h p$ approximation result.

Proposition 4.9. Let $K \in \mathcal{T}_{h}$ and suppose that $u \in H^{t_{K}}(K), t_{K} \geq 0$. Then there exists a sequence of polynomials $\pi_{p_{K}}^{h_{K}} u$ in $\mathcal{S}^{p_{K}}(K), p_{K}=1,2, \ldots$, satisfying

$$
\left\|u-\pi_{p_{K}}^{h_{K}} u\right\|_{q, K} \leq C \frac{h_{K}^{\min \left(p_{K}+1, t_{K}\right)-q}}{p_{K}^{t_{K}-q}}\|u\|_{t_{K}, K} \quad \forall 0 \leq q \leq t_{K} .
$$

Furthermore, if $t_{K} \geq 1$,

$$
\left\|u-\pi_{p_{K}}^{h_{K}} u\right\|_{0, \partial K} \leq C \frac{h_{K}^{\min \left(p_{K}+1, t_{K}\right)-\frac{1}{2}}}{p^{t_{K}-\frac{1}{2}}}\|u\|_{t_{K}, K} .
$$

The constant $C$ is independent of $u, h_{K}$ and $p_{K}$, but depends on the shape regularity of the mesh and on $t=\max _{K \in \mathcal{T}_{h}} t_{K}$.

Proof. The assertion (4.10) has been proved in 7, Lemma 4.5] for two-dimensional domains. For three-dimensional domains, the proof is analogous, see also 34 . In order to prove (4.11), we use the multiplicative trace inequality (see, e.g, [41, Lemma A.3])

$$
\|\eta\|_{0, \partial K}^{2} \leq C\left(\|\eta\|_{0, K}\|\nabla \eta\|_{0, K}+h_{K}^{-1}\|\eta\|_{0, K}^{2}\right)
$$

that holds true for any $\eta \in H^{1}(K)$ with a constant $C>0$ only depending on the shape regularity of the mesh. The second assertion now follows by applying in (4.12) the approximation result (4.10) for $q=0,1$.

We will denote by $\Pi_{p}^{h}$ the operator defined by $\left.\Pi_{p}^{h}(u)\right|_{K}=\pi_{p_{K}}^{h_{K}}\left(\left.u\right|_{K}\right)$, for any $K \in \mathcal{T}_{h}$, with $\pi_{p_{K}}^{h_{K}}\left(\left.u\right|_{K}\right)$ as in Proposition 4.9, and by $\boldsymbol{\Pi}_{p}^{h}$ the operator that maps $\mathbf{u}=\left(u_{1}, u_{2}, u_{3}\right)$ into $\left(\Pi_{p}^{h}\left(u_{1}\right), \Pi_{p}^{h}\left(u_{2}\right), \Pi_{p}^{h}\left(u_{3}\right)\right)$.

Next, we give an estimate of the residual $\mathcal{R}_{h}(\mathbf{u}, \mathbf{w}):=\mathcal{B}_{h}(\mathbf{u}, \mathbf{w})-\mathcal{F}_{h}(\mathbf{w})$.

Lemma 4.10. Let $\mathbf{u}$ be the exact solution. Assume $\left.\left(\mu^{-1} \nabla \times \mathbf{u}\right)\right|_{K} \in H^{s_{K}}(K)^{3}$, for all $K \in \mathcal{T}_{h}$, and $\left.(\nu \nabla \cdot(\varepsilon \mathbf{u}))\right|_{K} \in H^{s_{K}}(K)$, for all $K \in \mathcal{T}_{h}^{0}$, with local regularity exponents $s_{K} \geq 1$. Then, for any $\mathbf{w} \in \mathbf{V}_{h}$, the estimate

$$
\begin{aligned}
\left|\mathcal{R}_{h}(\mathbf{u}, \mathbf{w})\right| \leq C & \left(\sum_{K \in \mathcal{T}_{h}} \frac{h_{K}^{2 \min \left(p_{K}+1, s_{K}\right)} M_{K}}{p_{K}^{2 s_{K}}}\left\|\mu^{-1} \nabla \times \mathbf{u}\right\|_{s_{K}, K}^{2}\right)^{\frac{1}{2}}\|\mathbf{w}\|_{h} \\
& +C\left(\sum_{K \in \mathcal{T}_{h}^{0}} \frac{h_{K}^{2 \min \left(p_{K}+1, s_{K}\right)} M_{K}}{p_{K}^{2 s_{K}}}\|\nu \nabla \cdot(\varepsilon \mathbf{u})\|_{s_{K}, K}^{2}\right)^{\frac{1}{2}}\|\mathbf{w}\|_{h}
\end{aligned}
$$

hold true, where $M_{K}$ are the constants in (4.2). 
Proof. By straightforward calculations involving integration by parts, taking into account that $\mathbf{u} \in \mathbf{V}, \mathcal{L}\left(\llbracket \mathbf{u} \rrbracket_{T}\right)=\mathbf{G}_{\mathcal{D}}$, along with boundary and flux conditions, and the characterization of the data, we have that, for any $\mathbf{w} \in \mathbf{V}_{h}$,

$$
\begin{aligned}
\mathcal{R}_{h}(\mathbf{u}, \mathbf{w})= & \int_{\mathcal{E}}\left\{\left\{\mu^{-1} \nabla \times \mathbf{u}\right\} \cdot \llbracket \overline{\mathbf{w}} \rrbracket_{T} d s-\int_{\Omega} \mu^{-1} \nabla \times \mathbf{u} \cdot \mathcal{L}\left(\llbracket \overline{\mathbf{w}} \rrbracket_{T}\right) d \mathbf{x}\right. \\
& +\int_{\mathcal{E}_{\bar{I}}^{0}}\left\{\langle\nu \nabla \cdot(\varepsilon \mathbf{u})\} \llbracket \llbracket \varepsilon \overline{\mathbf{w}} \rrbracket_{N} d s-\int_{\Omega_{0}} \nu \nabla \cdot(\varepsilon \mathbf{u}) \mathcal{M}\left(\llbracket \varepsilon \overline{\mathbf{w}} \rrbracket_{N}\right) d \mathbf{x} .\right.
\end{aligned}
$$

Since

$$
\begin{aligned}
\int_{\Omega} \mu^{-1} \nabla \times \mathbf{u} \cdot \mathcal{L}\left(\llbracket \overline{\mathbf{w}} \rrbracket_{T}\right) d \mathbf{x} & =\int_{\Omega} \Pi_{\boldsymbol{\Sigma}_{h}}\left(\mu^{-1} \nabla \times \mathbf{u}\right) \cdot \mathcal{L}\left(\llbracket \overline{\mathbf{w}} \rrbracket_{T}\right) d \mathbf{x}, \\
\int_{\Omega_{0}} \nu \nabla \cdot(\varepsilon \mathbf{u}) \mathcal{M}\left(\llbracket \varepsilon \overline{\mathbf{w}} \rrbracket_{N}\right) d \mathbf{x} & =\int_{\Omega_{0}} \Pi_{Q_{h}}(\nu \nabla \cdot(\varepsilon \mathbf{u})) \mathcal{M}\left(\llbracket \varepsilon \overline{\mathbf{w}} \rrbracket_{N}\right) d \mathbf{x},
\end{aligned}
$$

for the $L^{2}$-projections $\boldsymbol{\Pi}_{\boldsymbol{\Sigma}_{h}}$ and $\Pi_{Q_{h}}$ onto $\boldsymbol{\Sigma}_{h}$ and $Q_{h}$, respectively, we can write

$$
\mathcal{R}_{h}(\mathbf{u}, \mathbf{w})=: T_{1}+T_{2}+T_{3}+T_{4},
$$

where

$$
\begin{aligned}
& T_{1}=\int_{\mathcal{E}}\left\{\mu^{-1} \nabla \times \mathbf{u}-\Pi_{\boldsymbol{\Sigma}_{h}}\left(\mu^{-1} \nabla \times \mathbf{u}\right)\right\} \cdot \llbracket \overline{\mathbf{w}} \rrbracket_{T} d s, \\
& T_{2}=\int_{\mathcal{E}_{\mathcal{I}}} \llbracket \mu^{-1} \nabla \times \mathbf{u}-\Pi_{\boldsymbol{\Sigma}_{h}}\left(\mu^{-1} \nabla \times \mathbf{u}\right) \rrbracket_{T} \cdot \mathrm{b} \llbracket \overline{\mathbf{w}} \rrbracket_{T} d s, \\
& T_{3}=\int_{\mathcal{E}_{I}^{0}}\left\{\nu \nabla \cdot(\varepsilon \mathbf{u})-\Pi_{Q_{h}}(\nu \nabla \cdot(\varepsilon \mathbf{u}))\right\} \llbracket \varepsilon \overline{\mathbf{w}} \rrbracket_{N} d s, \\
& T_{4}=\int_{\mathcal{E}_{I}^{0}} \llbracket \nu \nabla \cdot(\varepsilon \mathbf{u})-\Pi_{Q_{h}}(\nu \nabla \cdot(\varepsilon \mathbf{u})) \rrbracket \cdot \mathrm{d} \llbracket \varepsilon \overline{\mathbf{w}} \rrbracket_{N} d s .
\end{aligned}
$$

Let us bound the term $T_{1}$; the other terms are bounded similarly, observing our assumptions on $\mathrm{b}$ and $\mathrm{d}$. By the Cauchy-Schwarz and triangle inequalities, and the definition of $\|\cdot\|_{h}$, we obtain the bound

$$
\begin{aligned}
T_{1} \leq\|\mathbf{w}\|_{h}( & \left\|\mathrm{h}^{\frac{1}{2}} \mathbf{p}^{-1} \mathrm{~m}^{\frac{1}{2}}\left\{\left\{\mu^{-1} \nabla \times \mathbf{u}-\boldsymbol{\Pi}_{p}^{h}\left(\mu^{-1} \nabla \times \mathbf{u}\right)\right\}\right\}\right\|_{0, \mathcal{E}} \\
& +\| \mathrm{h}^{\frac{1}{2}} \mathrm{p}^{-1} \mathrm{~m}^{\frac{1}{2}}\left\{\left\{\boldsymbol{\Pi}_{p}^{h}\left(\mu^{-1} \nabla \times \mathbf{u}\right)-\boldsymbol{\Pi}_{\boldsymbol{\Sigma}_{h}}\left(\mu^{-1} \nabla \times \mathbf{u}\right)\right\} \|_{0, \mathcal{E}}\right) .
\end{aligned}
$$

From the definitions of $\mathrm{h}, \mathrm{p}$ and $\mathrm{m}$, and (4.11) with $t_{K}=s_{K}$, we conclude that

$$
\begin{aligned}
\| \mathrm{h}^{\frac{1}{2}} \mathrm{p}^{-1} \mathrm{~m}^{\frac{1}{2}} & \left\{\mu^{-1} \nabla \times \mathbf{u}-\mathbf{\Pi}_{p}^{h}\left(\mu^{-1} \nabla \times \mathbf{u}\right)\right\} \|_{0, \mathcal{E}}^{2} \\
& \leq C \sum_{K \in \mathcal{T}_{h}} \frac{h_{K} M_{K}}{p_{K}^{2}}\left\|\mu^{-1} \nabla \times \mathbf{u}-\boldsymbol{\Pi}_{p}^{h}\left(\mu^{-1} \nabla \times \mathbf{u}\right)\right\|_{0, \partial K}^{2} \\
& \leq C \sum_{K \in \mathcal{T}_{h}} \frac{h_{K}^{2 \min \left(p_{K}+1, s_{K}\right)} M_{K}}{p_{K}^{2 s_{K}+1}}\left\|\mu^{-1} \nabla \times \mathbf{u}\right\|_{s_{K}, K}^{2},
\end{aligned}
$$


and, similarly,

$$
\begin{aligned}
\| \mathrm{h}^{\frac{1}{2}} \mathrm{p}^{-1} \mathrm{~m}^{\frac{1}{2}} & \left\{\left\{\boldsymbol{\Pi}_{p}^{h}\left(\mu^{-1} \nabla \times \mathbf{u}\right)-\boldsymbol{\Pi}_{\boldsymbol{\Sigma}_{h}}\left(\mu^{-1} \nabla \times \mathbf{u}\right)\right\} \|_{0, \mathcal{E}}^{2}\right. \\
& \leq C \sum_{K \in \mathcal{T}_{h}} \frac{h_{K} M_{K}}{p_{K}^{2}}\left\|\boldsymbol{\Pi}_{p}^{h}\left(\mu^{-1} \nabla \times \mathbf{u}\right)-\boldsymbol{\Pi}_{\boldsymbol{\Sigma}_{h}}\left(\mu^{-1} \nabla \times \mathbf{u}\right)\right\|_{0, \partial K}^{2} \\
& \leq C \sum_{K \in \mathcal{T}_{h}} M_{K}\left\|\boldsymbol{\Pi}_{p}^{h}\left(\mu^{-1} \nabla \times \mathbf{u}\right)-\boldsymbol{\Pi}_{\boldsymbol{\Sigma}_{h}}\left(\mu^{-1} \nabla \times \mathbf{u}\right)\right\|_{0, K}^{2} \\
& \leq C \sum_{K \in \mathcal{T}_{h}} M_{K}\left\|\boldsymbol{\Pi}_{p}^{h}\left(\mu^{-1} \nabla \times \mathbf{u}\right)-\mu^{-1} \nabla \times \mathbf{u}\right\|_{0, K}^{2} \\
& \leq C \sum_{K \in \mathcal{T}_{h}} \frac{h_{K}^{2 \min \left(p_{K}+1, s_{K}\right)} M_{K}}{p_{K}^{s_{K}}}\left\|\mu^{-1} \nabla \times \mathbf{u}\right\|_{s_{K}, K}^{2},
\end{aligned}
$$

where we have used the inverse estimate (4.6), the fact that $\boldsymbol{\Pi}_{p}^{h}\left(\mu^{-1} \nabla \times \mathbf{u}\right)=$ $\boldsymbol{\Pi}_{\boldsymbol{\Sigma}_{h}} \boldsymbol{\Pi}_{p}^{h}\left(\mu^{-1} \nabla \times \mathbf{u}\right)$, the stability of the $L^{2}$-projection $\boldsymbol{\Pi}_{\boldsymbol{\Sigma}_{h}}$, and (4.10) with $t_{K}=s_{K}$ and $q=0$. Therefore, we obtain

$$
T_{1}^{2} \leq C\|w\|_{h}^{2} \sum_{K \in \mathcal{T}_{h}} \frac{h_{K}^{2 \min \left(p_{K}+1, s_{K}\right)} M_{K}}{p_{K}^{2 s_{K}}}\left\|\mu^{-1} \nabla \times \mathbf{u}\right\|_{s_{K}, K}^{2} .
$$

This, together with similar estimates for the terms $T_{2}, T_{3}$ and $T_{4}$ in the above expression for $\mathcal{R}_{h}(\mathbf{u}, \mathbf{w})$, proves the result.

In order to estimate the infimum at the right-hand side of the bound in Theorem 4.7 we make the assumption that the local meshsizes and approximation degrees have bounded variation; i.e., that there exist a constant $\ell>0$ such that

$$
\ell^{-1} h_{K} \leq h_{K^{\prime}} \leq \ell h_{K}, \quad \ell^{-1} p_{K} \leq p_{K^{\prime}} \leq \ell p_{K}
$$

for all $K$ and $K^{\prime}$ sharing a two-dimensional face. In particular, this assumption forbids the situation where the mesh is indefinitely refined in only one of two adjacent subdomains. Nevertheless, the above hypothesis is not restrictive in practice, and allows, for instance, for geometric refinement and linearly increasing approximation orders. For any element $K$, we define the quantities

$$
\begin{aligned}
m_{\delta K} & =\min \left\{m_{K^{\prime}}: K \text { and } K^{\prime} \text { share at least one face }\right\} \\
e_{\delta K} & =\max \left\{e_{K^{\prime}}: K \text { and } K^{\prime} \text { share at least one face }\right\}
\end{aligned}
$$

where $e_{K}$ denotes the maximum absolute value of the coefficients of $\left.\varepsilon\right|_{K}$, if $K \subset \Omega_{0}$, and is set for convenience to zero, if $K \subset \Omega_{\sigma}$.

We are now ready to prove the main approximation result.

Theorem 4.11. Assume the above hypotheses on $\mu, \nu, \varepsilon$ and on the coefficients in the definition of the numerical fluxes. Consider shape regular meshes and polynomial degree distributions obeying (4.14). Furthermore, denote by $\mathbf{u}_{h}$ the discrete solution of the LDG method defined in Section 3 and let the exact solution $\mathbf{u}$ satisfy $\left.\mathbf{u}\right|_{K} \in H^{s_{K}+1}(K)^{3},\left.\left(\mu^{-1} \nabla \times \mathbf{u}\right)\right|_{K} \in H^{s_{K}}(K)^{3}$, for all $K \in \mathcal{T}_{h}$, and $\left.(\nu \nabla \cdot(\varepsilon \mathbf{u}))\right|_{K} \in H^{s_{K}}(K)$, for all $K \in \mathcal{T}_{h}^{0}$, with local regularity exponents $s_{K} \geq 1$. 
Then we have the a priori error estimate

$$
\begin{aligned}
\left\|\mathbf{u}-\mathbf{u}_{h}\right\|_{h}^{2} \leq & C \sum_{K \in \mathcal{T}_{h}} \frac{h_{K}^{2 \min \left(p_{K}, s_{K}\right)}}{p_{K}^{2 s_{K}-1}}\left(N_{K}\|\mathbf{u}\|_{s_{K}+1, K}^{2}+M_{K}\left\|\mu^{-1} \nabla \times \mathbf{u}\right\|_{s_{K}, K}^{2}\right) \\
& +C \sum_{K \in \mathcal{T}_{h}^{0}} \frac{h_{K}^{2 \min \left(p_{K}, s_{K}\right)}}{p_{K}^{2 s_{K}-1}} M_{K}\|\nu \nabla \cdot(\varepsilon \mathbf{u})\|_{s_{K}, K}^{2},
\end{aligned}
$$

where $M_{K}$ are the constants in (4.2), $N_{K}=\frac{1+e_{\delta K}}{m_{\delta K}}+|\omega| \sup _{\mathbf{x} \in K}|\sigma(\mathbf{x})|+\lambda e_{\delta K}$, with $|\sigma(\mathbf{x})|$ denoting the spectral norm of the tensor $\sigma(\mathbf{x})$. The constant $C$ depends on $\Omega, \Omega_{0},\left\{s_{K}\right\}, \kappa, \ell, \alpha, \delta$, and on the shape regularity of the mesh, but is independent of the local meshsizes $h_{K}$ and the polynomial degrees $p_{K}$.

Proof. We start by estimating $\left\|\mathbf{u}-\boldsymbol{\Pi}_{p}^{h}(\mathbf{u})\right\|_{h}$, where $\boldsymbol{\Pi}_{p}^{h}$ is the operator defined after Proposition 4.9. From the definition of $\|\cdot\|_{h}$, the assumptions on $\mu, \nu, \varepsilon$ and on the coefficients in the definition of the numerical fluxes, and hypothesis (4.14), we have

$$
\begin{aligned}
\| \mathbf{u}- & \boldsymbol{\Pi}_{p}^{h} \mathbf{u} \|_{h}^{2} \leq C\left(\sum_{K \in \mathcal{T}_{h}} \frac{1+e_{\delta K}}{m_{\delta K}}\left\|\mathbf{u}-\boldsymbol{\Pi}_{p}^{h} \mathbf{u}\right\|_{1, K}^{2}+\sum_{K \in \mathcal{T}_{h}} \frac{p_{K}^{2}\left(1+e_{\delta K}\right)}{h_{K} m_{\delta K}}\left\|\mathbf{u}-\boldsymbol{\Pi}_{p}^{h} \mathbf{u}\right\|_{0, \partial K}^{2}\right. \\
& \left.+|\omega| \sum_{K \in \mathcal{T}_{h}} \sup _{\mathbf{x} \in K}|\sigma(\mathbf{x})|\left\|\mathbf{u}-\boldsymbol{\Pi}_{p}^{h} \mathbf{u}\right\|_{0, K}^{2}+\lambda e_{\delta K} \sum_{K \in \mathcal{T}_{h}^{0}: \partial K \cap \partial \Omega_{0} \neq \emptyset}\left\|\mathbf{u}-\boldsymbol{\Pi}_{p}^{h} \mathbf{u}\right\|_{0, \partial K}^{2}\right)
\end{aligned}
$$

( $C$ depends on $\Omega, \Omega_{0}, \kappa, \ell, \alpha, \delta$ and on the shape regularity of the mesh). The $h p$-approximation results with $t_{K}=s_{K}+1$ in Proposition 4.9 yield

$$
\left\|\mathbf{u}-\boldsymbol{\Pi}_{p}^{h} \mathbf{u}\right\|_{h}^{2} \leq C \sum_{K \in \mathcal{T}_{h}} \frac{h_{K}^{2 \min \left(p_{K}, s_{K}\right)}}{p_{K}^{2 s_{K}-1}} N_{K}\|\mathbf{u}\|_{s_{K}+1, K}^{2} .
$$

By inserting this and the estimate of Lemma 4.10 in the inequality of Theorem 4.7 we obtain the result.

Notice that for solutions $\mathbf{u} \in H^{s+1}\left(\mathcal{T}_{h}\right)^{3}$, with $\mu^{-1} \nabla \times \mathbf{u} \in H^{s}\left(\mathcal{T}_{h}\right)^{3}$ and $\nu \nabla \cdot(\varepsilon \mathbf{u}) \in H^{s}\left(\mathcal{T}_{h}^{0}\right), s \geq 1$, assuming constant approximation orders $p_{K}=p$ for all $K \in \mathcal{T}_{h}$, setting $h=\max _{K \in \mathcal{T}_{h}} h_{K}$, and incorporating bounds related to $\mu$, $\nu, \sigma, \varepsilon$ and $\lambda$ in the constant $C$, the estimate in Theorem 4.11 simply reads as

$$
\left\|\mathbf{u}-\mathbf{u}_{h}\right\|_{h} \leq C \frac{h^{\min (s, p)}}{p^{s-\frac{1}{2}}}\left(\|\mathbf{u}\|_{s+1, \mathcal{T}_{h}}+\left\|\mu^{-1} \nabla \times \mathbf{u}\right\|_{s, \mathcal{T}_{h}}+\|\nu \nabla \cdot(\varepsilon \mathbf{u})\|_{s, \mathcal{T}_{h}^{0}}\right) .
$$

This estimate is optimal in the meshsize $h$, and slightly suboptimal in $p$ (half a power of $p$ is lost). In the case of elliptic diffusion problems in two- or threedimensional domains, no better $p$-bound can be found in the literature for general unstructured grids (see, e.g., the $h p$-version analyses in [34, 41, 43]). Improved $p$-bounds have been obtained in [20] for one-dimensional convection-diffusion problems, and recently in [32] for two-dimensional reaction-diffusion problems on affine quadrilateral grids containing hanging nodes.

Remark 4.12. For solutions that are element-wise analytic, we have in fact exponential convergence as $p \rightarrow \infty$. This can be seen from the error bound in Theorem 4.7 and standard approximation properties for analytic functions (see, e.g., [44]). 
Note also that the restriction $s_{K} \geq 1$ has been made for convenience only, and it is possible to prove error estimates for $s_{K}>\frac{1}{2}$ as well. This minimal regularity assumption is still unrealistic when strong edge and corner singularities are present in the solutions (see [27]). On the other hand, the use of appropriate $h p$-mesh design principles might resolve these singularities at exponential convergence (see, e.g., 44). The extension of our analysis to such low-regularity cases remains to be done.

4.5. Error estimates for the auxiliary variables. By invoking the expressions in (3.11) and (3.12), we are able to derive error estimates for the auxiliary variables $\mathbf{s}$ and $\varphi$. This is important, in particular, because the variable $\mathbf{s}$ is related to the magnetic field that might be of interest. These estimates are a straightforward consequence of the following result.

Proposition 4.13. Under the same assumptions as in Theorem [4.11] we have

$$
\begin{aligned}
& \left\|\mu^{-1} \nabla \times \mathbf{u}-\mathbf{s}_{h}\right\|_{0, \Omega} \leq C\left(\left\|\mu^{-1} \nabla \times \mathbf{u}-\Pi_{p}^{h}\left(\mu^{-1} \nabla \times \mathbf{u}\right)\right\|_{0, \Omega}+\left\|\mathbf{u}-\mathbf{u}_{h}\right\|_{h}\right), \\
& \left\|\nu \nabla \cdot(\varepsilon \mathbf{u})-\varphi_{h}\right\|_{0, \Omega_{0}} \leq C\left(\left\|\nu \nabla \cdot(\varepsilon \mathbf{u})-\Pi_{p}^{h}(\nu \nabla \cdot(\varepsilon \mathbf{u}))\right\|_{0, \Omega_{0}}+\left\|\mathbf{u}-\mathbf{u}_{h}\right\|_{h}\right),
\end{aligned}
$$

with $C$ depending on $\alpha, \delta, \kappa$ and the shape regularity of the mesh.

Proof. Let us denote again by $\boldsymbol{\Pi}_{\boldsymbol{\Sigma}_{h}}$ the $L^{2}$-projection onto $\boldsymbol{\Sigma}_{h}$. Taking into account the identity (3.11), the triangle inequality, and that, for the exact solution $\mathbf{u}, \mathcal{L}\left(\llbracket \mathbf{u} \rrbracket_{T}\right)=\mathbf{G}_{\mathcal{D}}$, we obtain

$$
\left\|\mu^{-1} \nabla \times \mathbf{u}-\mathbf{s}_{h}\right\|_{0, \Omega} \leq T_{1}+T_{2},
$$

where

$$
\begin{aligned}
& T_{1}=\left\|\mu^{-1} \nabla \times \mathbf{u}-\boldsymbol{\Pi}_{\boldsymbol{\Sigma}_{h}}\left(\mu^{-1} \nabla_{h} \times \mathbf{u}_{h}\right)\right\|_{0, \Omega}, \\
& T_{2}=\left\|\boldsymbol{\Pi}_{\boldsymbol{\Sigma}_{h}}\left(\mu^{-1} \mathcal{L}\left(\llbracket \mathbf{u}-\mathbf{u}_{h} \rrbracket_{T}\right)\right)\right\|_{0, \Omega} .
\end{aligned}
$$

Using the stability of $\boldsymbol{\Pi}_{\boldsymbol{\Sigma}_{h}}$ and the estimates in Proposition 4.2 we obtain $T_{2} \leq$ $C\left\|\mathbf{u}-\mathbf{u}_{h}\right\|_{h}$. By using the the triangle inequality, the fact that $\Pi_{p}^{h}\left(\mu^{-1} \nabla \times \mathbf{u}\right)=$ $\boldsymbol{\Pi}_{\Sigma_{h}} \boldsymbol{\Pi}_{p}^{h}\left(\mu^{-1} \nabla \times \mathbf{u}\right)$, the stability of $\boldsymbol{\Pi}_{\Sigma_{h}}$, and the definition of $\|\cdot\|_{h}$, we estimate $T_{1}$ as

$$
\begin{aligned}
T_{1} & \leq\left\|\mu^{-1} \nabla \times \mathbf{u}-\boldsymbol{\Pi}_{\boldsymbol{\Sigma}_{h}}\left(\mu^{-1} \nabla \times \mathbf{u}\right)\right\|_{0, \Omega}+\left\|\boldsymbol{\Pi}_{\boldsymbol{\Sigma}_{h}}\left(\mu^{-1} \nabla \times \mathbf{u}-\mu^{-1} \nabla_{h} \times \mathbf{u}_{h}\right)\right\|_{0, \Omega} \\
& \leq C\left\|\mu^{-1} \nabla \times \mathbf{u}-\boldsymbol{\Pi}_{p}^{h}\left(\mu^{-1} \nabla \times \mathbf{u}\right)\right\|_{0, \Omega}+\left\|\mathbf{u}-\mathbf{u}_{h}\right\|_{h} .
\end{aligned}
$$

This completes the proof of the first estimate. The second one can be obtained in a similar way.

Proposition 4.13 together with Theorem 4.11 and Proposition 4.9 yields immediately $h p$-bounds for the error in $\mathbf{s}$ and $\varphi$. For instance, for solutions $\mathbf{u} \in H^{s+1}\left(\mathcal{T}_{h}\right)^{3}$, with $\mu^{-1} \nabla \times \mathbf{u} \in H^{s}\left(\mathcal{T}_{h}\right)^{3}$ and $\nu \nabla \cdot(\varepsilon \mathbf{u}) \in H^{s}\left(\mathcal{T}_{h}^{0}\right), s \geq 1$, and for constant approximation orders $p_{K}=p$, for all $K \in \mathcal{T}_{h}$, we get

$$
\begin{aligned}
& \left\|\mu^{-1} \nabla \times \mathbf{u}-\mathbf{s}_{h}\right\|_{0, \Omega} \leq C \frac{h^{\min (s, p)}}{p^{s-\frac{1}{2}}}\left(\|\mathbf{u}\|_{s+1, \mathcal{T}_{h}}+\left\|\mu^{-1} \nabla \times \mathbf{u}\right\|_{s, \mathcal{T}_{h}}+\|\nu \nabla \cdot(\varepsilon \mathbf{u})\|_{s, \mathcal{T}_{h}^{0}}\right), \\
& \left\|\nu \nabla \cdot(\varepsilon \mathbf{u})-\varphi_{h}\right\|_{0, \Omega_{0}} \leq C \frac{h^{\min (s, p)}}{p^{s-\frac{1}{2}}}\left(\|\mathbf{u}\|_{s+1, \mathcal{T}_{h}^{0}}+\left\|\mu^{-1} \nabla \times \mathbf{u}\right\|_{s, \mathcal{T}_{h}}+\|\nu \nabla \cdot(\varepsilon \mathbf{u})\|_{s, \mathcal{T}_{h}^{0}}\right) .
\end{aligned}
$$


4.6. Stability of discrete solutions. In this section, we investigate the stability of discrete solutions with respect to the data. First, we do this by proving a discrete Poincaré inequality, based on a duality argument similar to the one in [5]. We restrict ourselves to the case of $\mu=I$, with $I$ being the identity, since the elliptic regularity result needed for this argument does not hold true if $\mu$ is piecewise smooth (see [27]). Then, in the general case of discontinuous coefficients, the analogous stability result can be obtained, provided that the source term $\mathbf{F}$ satisfies certain restrictions.

Proposition 4.14. Assume that $\mu=I$, and take $\nu=1, \lambda=1$. Moreover, assume the above hypotheses (4.4) and (4.5) on the coefficients in the definition of the numerical fluxes. Then we have that

$$
\|\mathbf{v}\|_{0, \Omega} \leq C\|\mathbf{v}\|_{h}
$$

for any $\mathbf{v} \in H^{s}\left(\mathcal{T}_{h}\right)^{3}, s>\frac{1}{2}$. The constant $C$ is independent of the meshsizes and the approximation degrees.

Proof. For simplicity, we also assume that $\varepsilon=I$ (the case of piece-wise constant $\varepsilon$ being completely analogous). Fix $\mathbf{v} \in H^{s}\left(\mathcal{T}_{h}\right)^{3}$. Since $\mathbf{v} \in L^{2}(\Omega)^{3}$, we can decompose $\mathbf{v}$ according to (2.6) into $\mathbf{v}=\mathbf{F}^{\prime}+\mathbf{F}^{\prime \prime}$, with $\mathbf{F}^{\prime \prime}=\nabla f$ in $\Omega_{0}$ and $f=f_{j}$ on $\Gamma_{0, j}, j=1, \ldots, J$. We consider the following dual problem. Find $\mathbf{z} \in H(\operatorname{curl} ; \Omega) \cap H\left(\operatorname{div} ; \Omega_{0}\right)$ such that

$$
\begin{array}{ll}
\nabla \times \nabla \times \mathbf{z}+i \omega \sigma \mathbf{z}=\mathbf{F}^{\prime} & \text { in } \Omega, \\
\nabla \cdot \mathbf{z}=-f & \text { in } \Omega_{0}, \\
\mathbf{n} \times \mathbf{z}=\mathbf{0} & \text { on } \partial \Omega, \\
\left\langle\left.\mathbf{z}\right|_{\Omega_{0}} \cdot \mathbf{n}_{0, j}, 1\right\rangle_{\Gamma_{0, j}}=f_{j} & \forall j=1, \ldots, J .
\end{array}
$$

First, we claim that

$$
\nabla \times \mathbf{z} \in H^{s_{0}}(\Omega)^{3}, \quad\|\nabla \times \mathbf{z}\|_{s_{0}, \Omega} \leq C\|\mathbf{v}\|_{0, \Omega}, \quad \text { for } s_{0}>\frac{1}{2} .
$$

To prove this, set $\mathbf{w}=\nabla \times \mathbf{z}$. From equation (4.15), we have $\nabla \times \mathbf{w}=-i \omega \sigma \mathbf{z}+\mathbf{F}^{\prime} \in$ $L^{2}(\Omega)^{3}$. Furthermore, $\nabla \cdot \mathbf{w}=0$ and $\mathbf{w} \cdot \mathbf{n}=\nabla \times \mathbf{z} \cdot \mathbf{n}=0$ on $\partial \Omega$. Hence, from [4, Proposition 3.7], it follows that $\mathbf{w} \in H^{s_{0}}(\Omega)^{3}$ for a regularity exponent $s_{0}>\frac{1}{2}$, as well as $\|\mathbf{w}\|_{s_{0}, \Omega} \leq C\|\mathbf{w}\|_{H(\operatorname{curl} ; \Omega)}$. However, $\|\mathbf{w}\|_{H(\operatorname{curl} ; \Omega)} \leq C\|\mathbf{z}\|_{H(\operatorname{curl} ; \Omega)}+\left\|\mathbf{F}^{\prime}\right\|_{0, \Omega}$. Then, from the stability estimate in Theorem 2.1 and the $L^{2}$-orthogonality of the decomposition of $\mathbf{v}$, we also have $\|\mathbf{w}\|_{s_{0}, \Omega} \leq C\|\mathbf{v}\|_{0, \Omega}$, which completes the proof of (4.19).

Subtracting the gradient of equation (4.16) from (4.15), multiplying the result by $\overline{\mathbf{v}}$, and integrating over $\Omega$ and $\Omega_{0}$, owing to the decomposition of $\mathbf{v}$, we obtain that

$$
\|\mathbf{v}\|_{0, \Omega}^{2}=\int_{\Omega}(\nabla \times \nabla \mathbf{z}+i \omega \sigma \mathbf{z}) \cdot \overline{\mathbf{v}} d \mathbf{x}-\int_{\Omega_{0}}(\nabla \nabla \cdot \mathbf{z}) \cdot \overline{\mathbf{v}} d \mathbf{x} .
$$

Let us first consider the integral containing the curl-curl term. Integration by parts, together with the first rule in (3.9), as well as the Cauchy-Schwarz inequality and 
the definition of $\|\cdot\|_{h}$, gives

$$
\begin{aligned}
\left|\int_{\Omega} \nabla \times \nabla \mathbf{z} \cdot \overline{\mathbf{v}} d \mathbf{x}\right| & \leq\left|\int_{\Omega} \nabla \times \mathbf{z} \cdot \nabla_{h} \times \overline{\mathbf{v}} d \mathbf{x}\right|+\left|\int_{\mathcal{E}}\{\nabla \times \mathbf{z}\} \cdot \llbracket \overline{\mathbf{v}} \rrbracket_{T} d s\right| \\
& \leq\|\mathbf{z}\|_{H(\operatorname{curl} ; \Omega)}\|\mathbf{v}\|_{h}+C\left(\sum_{K \in \mathcal{T}_{h}} \frac{h_{K}}{p_{K}^{2}}\|\nabla \times \mathbf{z}\|_{0, \partial K}^{2}\right)^{\frac{1}{2}}\|\mathbf{v}\|_{h} .
\end{aligned}
$$

Using similar scaling arguments as the ones in [3, Lemma 5.2], together with the result of [3. Lemma 5.5], we can see that

$$
\|\nabla \times \mathbf{z}\|_{0, \partial K}^{2} \leq C h_{K}^{-1}\|\nabla \times \mathbf{z}\|_{s_{0}, K}^{2},
$$

with a constant $C$ only depending on the shape regularity of the meshes. This, together with $p_{K}^{-1} \leq 1$, yields $\sum_{K \in \mathcal{T}_{h}} \frac{h_{K}}{p_{K}^{2}}\|\nabla \times \mathbf{z}\|_{0, \partial K}^{2} \leq C\|\nabla \times \mathbf{z}\|_{s_{0}, \Omega} \leq C\|\mathbf{v}\|_{0, \Omega}$. Therefore, since also $\|\mathbf{z}\|_{H(\operatorname{curl} ; \Omega)} \leq C\|\mathbf{v}\|_{0, \Omega}$ (see Theorem 2.1), we get

$$
\left|\int_{\Omega} \nabla \times \nabla \mathbf{z} \cdot \overline{\mathbf{v}} d \mathbf{x}\right| \leq C\|\mathbf{v}\|_{0, \Omega}\|\mathbf{v}\|_{h}
$$

Similarly, by integration by parts, using the second identity in (3.9), we obtain

$$
\begin{aligned}
\left|\int_{\Omega_{0}}(\nabla \nabla \cdot \mathbf{z}) \cdot \overline{\mathbf{v}} d \mathbf{x}\right| \leq\|\mathbf{z}\|_{H\left(\operatorname{div} ; \Omega_{0}\right)}\|\mathbf{v}\|_{h} & \\
& +\left|\int_{\mathcal{E}_{\mathcal{I}}^{0}}\{\{\nabla \cdot \mathbf{z}\}\} \llbracket \overline{\mathbf{v}} \rrbracket_{N} d s\right|+\sum_{j=1}^{J}\left|f_{j}\right| \int_{\Gamma_{0, j}}\left|\overline{\mathbf{v}} \cdot \mathbf{n}_{0, j}\right| d s,
\end{aligned}
$$

where we also used the fact that $\nabla \cdot \mathbf{z}$ is zero on $\Gamma_{0,0}$ and equal to $-f_{j}$ on $\Gamma_{0, j}$, for $j=1, \ldots, J$. Since $\|\nabla \cdot \mathbf{z}\|_{0, \partial K} \leq C h_{K}^{-\frac{1}{2}}\|\nabla \cdot \mathbf{z}\|_{1, K}$, for all $K \in \mathcal{T}_{h}^{0}$, we get

$$
\left|\int_{\mathcal{E}_{\mathcal{I}}^{0}}\{\{\nabla \cdot \mathbf{z}\}\} \llbracket \overline{\mathbf{v}} \rrbracket_{N} d s\right| \leq C\|\nabla \cdot \mathbf{z}\|_{1, \Omega_{0}}\|\mathbf{v}\|_{h} .
$$

The Cauchy-Schwarz inequality, the trace theorem, and the standard Poincaré inequality then yield

$$
\sum_{j=1}^{J}\left|f_{j}\right| \int_{\Gamma_{0, j}}\left|\overline{\mathbf{v}} \cdot \mathbf{n}_{0, j}\right| d s \leq C\|\nabla f\|_{0, \Omega_{0}}\|\mathbf{v}\|_{h} .
$$

Combining the above estimates, together with $\|\mathbf{z}\|_{H\left(\text { div } ; \Omega_{0}\right)}+\|\nabla \cdot \mathbf{z}\|_{1, \Omega_{0}} \leq C\|\mathbf{v}\|_{0, \Omega}$ and a similar argument for the term $i \omega \int_{\Omega} \sigma \mathbf{z} \overline{\mathbf{v}} d \mathbf{x}$, shows that

$$
\|\mathbf{v}\|_{0, \Omega}^{2} \leq C\|\mathbf{v}\|_{0, \Omega}\|\mathbf{v}\|_{h} .
$$

This completes the proof.

From Proposition 4.14, we are able to establish the continuity of the functional $\mathcal{F}_{h}(\cdot)$ in (3.13) with respect to the norm $\|\cdot\|_{h}$, at least in the case where $\mu=I$.

Corollary 4.15. Under the same assumptions as in Proposition 4.14, we have for all $\mathbf{v} \in \mathbf{V}_{h}$,

$$
\left|\mathcal{F}_{h}(\mathbf{v})\right| \leq C\left(\|\mathbf{F}\|_{0, \Omega}^{2}+\left\|\mathbf{h}^{-\frac{1}{2}} \mathbf{p} \mathbf{g}\right\|_{0, \partial \Omega}^{2}\right)^{\frac{1}{2}}\|\mathbf{v}\|_{h},
$$

with a constant $C$ independent of the meshsizes and the approximation degrees. 
Proof. From the definition of $\mathcal{F}_{h}$, Proposition 4.14 and the Cauchy-Schwarz inequality, we obtain

$$
\left|\mathcal{F}_{h}(\mathbf{v})\right| \leq C\left(\|\mathbf{F}\|_{0, \Omega}^{2}+\left\|\mathbf{G}_{\mathcal{D}}\right\|_{0, \Omega}^{2}+\left\|\mathrm{h}^{-\frac{1}{2}} \mathbf{p} \mathbf{g}\right\|_{0, \partial \Omega}^{2}\right)^{\frac{1}{2}}\|\mathbf{v}\|_{h},
$$

for any $\mathbf{v} \in \mathbf{V}_{h}$. The result follows from the estimate for $\mathbf{G}_{\mathcal{D}}$ in Proposition 4.2.

Consider now the case where $\mu$ satisfies our more general assumptions. The part of the functional $\mathcal{F}_{h}$ involving the boundary term can be dealt with as in Corollary 4.15 therefore, we will focus on the term $\int_{\Omega} \mathbf{F} \cdot \overline{\mathbf{v}} d \mathbf{x}$. We can prove the following result.

Proposition 4.16. Assume our general hypotheses on $\mu, \nu, \varepsilon$ and the coefficients in the definition of the numerical fluxes. Whenever $\Omega_{0}$ is not simply connected, let $\left\{\Sigma_{\ell}\right\}_{\ell=1, \ldots, L}$ be an admissible set of cuts for $\Omega_{0}$ in the sense of [4, and denote by $\mathbf{n}_{\ell}$ the normal unit vector to $\Sigma_{\ell}$, pointing in one of the two possible directions. Given $\mathbf{F} \in L^{2}(\Omega)^{3}$, let $\varepsilon^{-1} \mathbf{F}=\mathbf{F}^{\prime}+\mathbf{F}^{\prime \prime}$ be its orthogonal decomposition, according to (2.6). If

$$
\begin{array}{ll}
\varepsilon \mathbf{F}^{\prime} \cdot \mathbf{n}_{0}=0 & \text { on } \partial \Omega_{0}, \\
\left\langle\varepsilon \mathbf{F}^{\prime} \cdot \mathbf{n}_{\ell}, 1\right\rangle_{\Sigma_{\ell}}=0 & \forall \ell=1, \ldots, L,
\end{array}
$$

then, for all $\mathbf{v} \in \mathbf{V}_{h}$, we have

$$
\int_{\Omega} \mathbf{F} \cdot \overline{\mathbf{v}} d \mathbf{x} \leq C\|\mathbf{F}\|_{0, \Omega}\|\mathbf{v}\|_{h}
$$

with a constant $C$ independent of the meshsizes and the approximation degrees.

We remark that, if $\mathbf{F}=\mathbf{J}$, then (4.20) is satisfied $\left(\varepsilon \mathbf{F}^{\prime}=\mathbf{J}\right.$ and $\left.\mathbf{F}^{\prime \prime}=\mathbf{0}\right)$. Moreover, whenever $\Omega_{0}$ is simply connected, the second condition in (4.20) is empty.

Proof of Proposition 4.16. Recall that in the orthogonal decomposition (2.6) of $\varepsilon^{-1} \mathbf{F}=\mathbf{F}^{\prime}+\mathbf{F}^{\prime \prime}$, the function $\mathbf{F}^{\prime \prime}$ is such that $\left.\mathbf{F}^{\prime \prime}\right|_{\Omega_{\sigma}}=\mathbf{0}$ and $\left.\mathbf{F}^{\prime \prime}\right|_{\Omega_{0}}=\nabla f$, with $f \in H^{1}\left(\Omega_{0}\right), f=0$ on $\Gamma_{0,0}$ and $f$ constant, say $f=f_{j}$, on each $\Gamma_{0, j}$ for $j=1, \ldots, J$. The standard Poincaré inequality implies that $\|f\|_{0, \Omega_{0}}$ and $\|f\|_{1, \Omega_{0}}$ are equivalent to $\|\nabla f\|_{0, \Omega_{0}}=\left\|\mathbf{F}^{\prime \prime}\right\|_{0, \Omega_{0}}$. From [4, Theorem 3.17], the assumptions (4.20) on $\mathbf{F}^{\prime}$, together with $\left.\nabla \cdot\left(\varepsilon \mathbf{F}^{\prime}\right)\right|_{\Omega_{0}}=0$, imply that there exists $\mathbf{w} \in H_{0}\left(\right.$ curl, div; $\left.\Omega_{0}\right)$ with $\nabla \cdot \mathbf{w}=0$ in $\Omega_{0}$ and $\left\langle\mathbf{w} \cdot \mathbf{n}_{0, j}, 1\right\rangle_{\Gamma_{0, j}}=0, j=1, \ldots, J$, such that $\varepsilon \mathbf{F}^{\prime}=\nabla \times \mathbf{w}$. Corollary 3.19 in 4 implies that $\|\mathbf{w}\|_{0, \Omega_{0}}$ and $\|\mathbf{w}\|_{H\left(\mathrm{curl} ; \Omega_{0}\right)}$ are equivalent to $\|\nabla \times \mathbf{w}\|_{0, \Omega_{0}}=\left\|\varepsilon \mathbf{F}^{\prime}\right\|_{0, \Omega_{0}}$. Moreover, from the continuous imbedding of $H_{0}\left(\right.$ curl, div; $\left.\Omega_{0}\right)$ in $H^{s}\left(\Omega_{0}\right)^{3}$, for some $s>\frac{1}{2}$, we also have that $\mathbf{w} \in H^{s}\left(\Omega_{0}\right)^{3}$ and $\|\mathbf{w}\|_{s, \Omega_{0}}$ can be controlled by $\|\mathbf{w}\|_{H\left(\operatorname{curl} ; \Omega_{0}\right)}$.

After these preliminaries, we can proceed by estimating $\int_{\Omega} \mathbf{F} \cdot \overline{\mathbf{v}} d \mathbf{x}$. We can write

$$
\begin{aligned}
\int_{\Omega} \mathbf{F} \cdot \overline{\mathbf{v}} d \mathbf{x}= & \int_{\Omega_{\sigma}} \mathbf{F} \cdot \overline{\mathbf{v}} d \mathbf{x}+\int_{\Omega_{0}} \nabla \times \mathbf{w} \cdot \overline{\mathbf{v}} d \mathbf{x}+\int_{\Omega_{0}} \nabla f \cdot(\varepsilon \overline{\mathbf{v}}) d \mathbf{x} \\
= & \int_{\Omega_{\sigma}} \mathbf{F} \cdot \overline{\mathbf{v}} d \mathbf{x}+\int_{\Omega_{0}} \mathbf{w} \cdot \nabla_{h} \times \overline{\mathbf{v}} d \mathbf{x}-\sum_{K \in \mathcal{T}_{h}^{0}} \int_{\partial K} \mathbf{w} \cdot \mathbf{n}_{K} \times \overline{\mathbf{v}} d s \\
& -\int_{\Omega_{0}} f \nabla_{h} \cdot(\varepsilon \overline{\mathbf{v}}) d \mathbf{x}+\sum_{K \in \mathcal{T}_{h}^{0}} \int_{\partial K} f \varepsilon \overline{\mathbf{v}} \cdot \mathbf{n}_{K} d s .
\end{aligned}
$$


Therefore,

$$
\begin{aligned}
\int_{\Omega} \mathbf{F} \cdot \overline{\mathbf{v}} & d \mathbf{x} \leq|\omega|^{-1}\left\|\sigma^{-\frac{1}{2}} \mathbf{F}\right\|_{0, \Omega_{\sigma}}|\omega|\left\|\sigma^{\frac{1}{2}} \mathbf{v}\right\|_{0, \Omega_{\sigma}}+\left\|\mu^{\frac{1}{2}} \mathbf{w}\right\|_{0, \Omega_{0}}\left\|\mu^{-\frac{1}{2}} \nabla_{h} \times \mathbf{v}\right\|_{0, \Omega_{0}} \\
& +\left\|\mathrm{h}^{\frac{1}{2}} \mathrm{p}^{-1} \mathrm{~m}^{\frac{1}{2}} \mathbf{w}\right\|_{0, \mathcal{E}_{I}^{0}}\left\|\mathrm{~h}^{-\frac{1}{2}} \mathrm{pm}^{-\frac{1}{2}} \llbracket \mathbf{v} \rrbracket_{T}\right\|_{0, \mathcal{E}_{I}^{0}}+\left\|\nu^{-\frac{1}{2}} f\right\|_{0, \Omega_{0}}\left\|\nu^{\frac{1}{2}} \nabla_{h} \cdot(\varepsilon \mathbf{v})\right\|_{0, \Omega_{0}} \\
& +\left\|\mathrm{h}^{\frac{1}{2}} \mathrm{p}^{-1} \mathrm{~m}^{\frac{1}{2}} f\right\|_{0, \mathcal{E}_{I}^{0}}\left\|\mathrm{~h}^{-\frac{1}{2}} \mathrm{pm}^{-\frac{1}{2}} \llbracket \varepsilon \mathbf{v} \rrbracket_{N}\right\|_{0, \mathcal{E}_{I}^{0}} \\
& +\left(\lambda^{-1} \sum_{j=1}^{J} f_{j}^{2}\right)^{\frac{1}{2}}\left(\lambda \sum_{j=1}^{J}\left|\left\langle\varepsilon \mathbf{v} \cdot \mathbf{n}_{0, j}, 1\right\rangle_{\Gamma_{0, j}}\right|^{2}\right)^{\frac{1}{2}}
\end{aligned}
$$

where we have used the fact that $\mathbf{n}_{0} \times \mathbf{w}=\mathbf{0}$ on $\partial \Omega_{0}$. Consequently,

$$
\begin{array}{r}
\int_{\Omega} \mathbf{F} \cdot \overline{\mathbf{v}} d \mathbf{x} \leq C\|\mathbf{v}\|_{h}\left(|\omega|^{-1}\left\|\sigma^{-\frac{1}{2}} \mathbf{F}\right\|_{0, \Omega_{\sigma}}^{2}+\left\|\mu^{\frac{1}{2}} \mathbf{w}\right\|_{0, \Omega_{0}}^{2}+\left\|\mathrm{h}^{\frac{1}{2}} \mathrm{p}^{-1} \mathrm{~m}^{\frac{1}{2}} \mathbf{w}\right\|_{0, \mathcal{E}^{0}}^{2}\right. \\
\left.+\left\|\nu^{-\frac{1}{2}} f\right\|_{0, \Omega_{0}}^{2}+\left\|\mathrm{h}^{\frac{1}{2}} \mathrm{p}^{-1} \mathrm{~m}^{\frac{1}{2}} f\right\|_{0, \mathcal{E}_{I}^{0}}^{2}+\lambda^{-1} \sum_{j=1}^{J} f_{j}^{2}\right) .
\end{array}
$$

For the volume terms at right-hand side, we have

$$
\begin{aligned}
& |\omega|^{-1}\left\|\sigma^{-\frac{1}{2}} \mathbf{F}\right\|_{0, \Omega_{\sigma}}^{2} \leq C\|\mathbf{F}\|_{0, \Omega_{\sigma}}^{2}, \\
& \left\|\mu^{\frac{1}{2}} \mathbf{w}\right\|_{0, \Omega_{0}}^{2} \leq C\|\mathbf{w}\|_{0, \Omega_{0}}^{2} \leq C\|\mathbf{F}\|_{0, \Omega_{0}}^{2}, \\
& \left\|\nu^{-\frac{1}{2}} f\right\|_{0, \Omega_{0}}^{2} \leq C\|f\|_{0, \Omega_{0}}^{2} \leq C\|\mathbf{F}\|_{0, \Omega_{0}}^{2},
\end{aligned}
$$

with $C$ independent of the meshsizes and the approximation degrees. Using standard scaling arguments, we obtain

$$
\begin{aligned}
\left\|\mathrm{h}^{\frac{1}{2}} \mathrm{p}^{-1} \mathrm{~m}^{\frac{1}{2}} \mathbf{w}\right\|_{0, \mathcal{E}^{0}}^{2} & \leq \sum_{K \in \mathcal{T}_{h}^{0}} \frac{h_{K} M_{K}}{p_{K}}\|\mathbf{w}\|_{0, \partial K}^{2} \leq \sum_{K \in \mathcal{T}_{h}^{0}} \frac{h_{K} M_{K}}{p_{K}} h_{K}^{-1}\|\mathbf{w}\|_{s, K}^{2} \\
& \leq C\|\mathbf{w}\|_{s, \Omega_{0}}^{2} \leq C\|\mathbf{w}\|_{H\left(\operatorname{curl} ; \Omega_{0}\right)}^{2} \leq C\|\mathbf{F}\|_{0, \Omega_{0}}^{2},
\end{aligned}
$$

again with $C$ independent of the meshsizes and the approximation degrees. Similarly,

$$
\begin{aligned}
\left\|\mathrm{h}^{\frac{1}{2}} \mathrm{p}^{-1} \mathrm{~m}^{\frac{1}{2}} f\right\|_{0, \mathcal{E}_{I}^{0}}^{2} & \leq \sum_{K \in \mathcal{T}_{h}^{0}} \frac{h_{K} M_{K}}{p_{K}}\|f\|_{0, \partial K}^{2} \leq \sum_{K \in \mathcal{T}_{h}^{0}} \frac{h_{K} M_{K}}{p_{K}} h_{K}^{-1}\|f\|_{1, K}^{2} \\
& \leq C\|f\|_{1, \Omega_{0}}^{2} \leq C\|F\|_{0, \Omega_{0}}^{2},
\end{aligned}
$$

and

$$
\lambda^{-1} \sum_{j=1}^{J} f_{j}^{2} \leq C \lambda^{-1}\|f\|_{1, \Omega_{0}} \leq C\|\mathbf{F}\|_{0, \Omega_{0}} .
$$

This completes the proof of the proposition.

Remark 4.17. In the particular case where $\Omega=\Omega_{0}$ (and $\Omega_{\sigma}=\emptyset$ ), the stability result of Proposition 4.16 holds true without any restriction on $\mathbf{F}$. In fact, the aim of assumption (4.20) is to guarantee the condition $\mathbf{n}_{0} \times \mathbf{w}=\mathbf{0}$ on $\partial \Omega_{0}$ for $\mathbf{w}$ in the expression $\varepsilon \mathbf{F}^{\prime}=\nabla \times \mathbf{w}$, which is needed in order to derive the estimates in the proof of Proposition 4.16 As can be inferred from the proof, this condition is no longer necessary if $\Omega=\Omega_{0}$. niIn this case, we can use [4, Theorem 3.12] instead of [4] Theorem 3.17] and express $\varepsilon \mathbf{F}^{\prime}$ as $\nabla \times \mathbf{w}$, with $\mathbf{w} \in H(\operatorname{curl} ; \Omega)$; the norm 
equivalence is established by [4, Corollary 3.16] instead of [4, Corollary 3.19]. Then the proof goes through without the condition $\mathbf{n} \times \mathbf{w}=\mathbf{0}$ on $\partial \Omega=\partial \Omega_{0}$.

\section{Conclusions and extensions}

In this paper, a local discontinuous Galerkin method for the discretization of the time-harmonic Maxwell equations in low-frequency regime has been proposed and its $h p$-error analysis has been carried out. Heterogeneous materials and topologically nontrivial domains have been considered; assumptions that are realistic in practice. Although the setting of [6] to cast the LDG method in its primal form has been used, a new technique to derive error estimates has been proposed, which is based on Strang's lemma and which might be of independent interest in the analysis of DG methods. For triangulations containing hanging nodes, $h p$-error estimates that are optimal in the meshsize and suboptimal in the approximation order have been derived. This analysis is the first $h p$-error analysis for the LDG method in several space dimensions and in this sense also extends our previous work in [19].

Let us also indicate some related issues that are still open:

- For the Laplacian, the parameters b and d can be chosen in such a way that the LDG method superconverges on Cartesian grids and for tensor product polynomials (see [24]). Whether or not a similar phenomenon takes place in the context of the $p$-version of the method is an open question and has to be addressed in future work.

- The study of $h p$-refinement toward edge and corner singularities, in order to resolve them at exponential convergence, remains an open problem.

- One of the drawbacks of discontinuous Galerkin methods is the relatively high number of degrees of freedom due to the discontinuous nature of the finite element spaces. This problem can be overcome by coupling discontinuous and conforming elements, following [40]. The approach there combines the ease with which the LDG method handles hanging nodes with the lower computational cost of standard finite elements.

- The extension of the LDG method to mixed boundary conditions can be done in a straightforward way (see, e.g, [19]). However, in order to avoid further complications in the analysis of the corresponding continuous problems, this point is omitted in this paper.

We conclude by pointing out that the analysis of discontinuous Galerkin methods for time-harmonic Maxwell equations in the high-frequency case as well as the numerical study and implementation of our methods are the subject of ongoing work.

\section{ACKNOWLEDGMENTS}

The authors would like to thank Ana Alonso for useful discussions on the lowfrequency model (2.1)-(2.4) for the heterogeneous time-harmonic Maxwell equations.

\section{Appendix A. The proof of Theorem 2.1}

This appendix is devoted to the proof of Theorem 2.1. We start by establishing, in subsection A.1 the existence of a continuous and $\varepsilon$-divergence-free lifting of tangential traces. This result is a key tool in the proof of existence and uniqueness of solutions to our model problem which is developed in subsection A.2 
A.1. Divergence-free continuous lifting of tangential traces. Recall that we denote by $\mathcal{H}(\partial \Omega)$ the space of tangential traces of $H(\operatorname{curl} ; \Omega)$-functions. We refer to [15] for its complete characterization and for the definition of its norm. The existence of a continuous lifting of the tangential traces of $H(\operatorname{curl} ; \Omega)$-functions is guaranteed by Theorem 3.1 in 14 in the case where $\Omega$ is a bounded, open Lipschitz polyhedron in $\mathbb{R}^{3}$, not necessarily simply connected. In this section, we show that we can actually require this lifting to have zero $\varepsilon$-divergence in a Lipschitz polyhedral subdomain $\Omega_{0}$ of $\Omega$, and to satisfy homogeneous flux conditions through surfaces of the possible cavities of $\Omega_{0}$. This result is stated in the following proposition. We define the space

$$
H_{\text {flux }}\left(\operatorname{div}_{\varepsilon} ; \Omega_{0}\right)=\left\{\mathbf{v} \in H\left(\operatorname{div}_{\varepsilon} ; \Omega_{0}\right):\left\langle\left.\varepsilon \mathbf{v}\right|_{\Omega_{0}} \cdot \mathbf{n}_{0, j}, 1\right\rangle_{\Gamma_{0, j}}=0, j=1, \ldots, J\right\},
$$

along with its subspace $H_{\text {flux }}\left(\operatorname{div}_{\varepsilon}^{0} ; \Omega_{0}\right)=H_{\text {flux }}\left(\operatorname{div}_{\varepsilon} ; \Omega_{0}\right) \cap H\left(\operatorname{div}_{\varepsilon}^{0} ; \Omega_{0}\right)$.

Proposition A.1. Let $\Omega$ be a connected, bounded, open Lipschitz polyhedron in $\mathbb{R}^{3}$, and $\Omega_{0} \subseteq \Omega$ a Lipschitz polyhedral subdomain. Then, given $\widetilde{\mathbf{g}} \in H(\operatorname{curl} ; \Omega)$, there is $\widetilde{\mathbf{u}} \in H(\operatorname{curl} ; \Omega) \cap H_{\text {flux }}\left(\operatorname{div}_{\varepsilon}^{0} ; \Omega_{0}\right)$ such that $\mathbf{n} \times \widetilde{\mathbf{u}}=\mathbf{n} \times \widetilde{\mathbf{g}}=: \mathbf{g}$ on $\partial \Omega$ and $\|\widetilde{\mathbf{u}}\|_{H(\operatorname{curl} ; \Omega)} \leq C\|\mathbf{g}\|_{\mathcal{H}(\partial \Omega)}$.

In order to prove Proposition A.1, we need the following two lemmas.

Lemma A.2. Let $\Omega$ be a connected, bounded, open Lipschitz polyhedron in $\mathbb{R}^{3}$. Then, given $\widetilde{\mathbf{g}} \in H(\operatorname{curl} ; \Omega)$, there is $\widetilde{\mathbf{u}}_{\mathrm{bc}} \in H(\operatorname{curl} ; \Omega) \cap H\left(\operatorname{div}_{\varepsilon}^{0} ; \Omega\right)$ such that $\mathbf{n} \times$ $\widetilde{\mathbf{u}}_{\mathrm{bc}}=\mathbf{n} \times \widetilde{\mathbf{g}}=: \mathbf{g}$ on $\partial \Omega$ and $\left\|\widetilde{\mathbf{u}}_{\mathrm{bc}}\right\|_{H(\mathrm{curl} ; \Omega)} \leq C\|\mathbf{g}\|_{\mathcal{H}(\partial \Omega)}$.

Proof. Let $\widetilde{\mathbf{g}} \in H(\operatorname{curl} ; \Omega)$. Since $\nabla \times H(\operatorname{curl} ; \Omega)=\nabla \times H^{1}(\Omega)^{3}$ (see [30]), there exists $\mathbf{g}_{1} \in H^{1}(\Omega)^{3}$ such that $\nabla \times \mathbf{g}_{1}=\nabla \times \widetilde{\mathbf{g}}$. Then, we can write $\widetilde{\mathbf{g}}=\mathbf{g}_{1}+\mathbf{g}_{2}$, with $\mathbf{g}_{2} \in H(\operatorname{curl} ; \Omega)$ and $\nabla \times \mathbf{g}_{2}=0$. Consequently, $\mathbf{g}_{2}$ admits the $\varepsilon$-orthogonal decomposition $\mathbf{g}_{2}=\nabla \varphi+\mathbf{w}$, with $\varphi \in H^{1}(\Omega)$, w $\in H_{0}\left(\operatorname{div}_{\varepsilon}^{0} ; \Omega\right) \cap H\left(\operatorname{curl}^{0} ; \Omega\right)$, (see [30], formula (4.14) with $\Gamma_{\nu}=\partial \Omega, \Gamma_{\tau}=\emptyset$, and $\omega=\varepsilon$ ).

Let $\widetilde{\varphi}$ be the unique solution to the following problem: Find $\widetilde{\varphi} \in H^{1}(\Omega)^{3}$ such that $\widetilde{\varphi}=\left.\varphi\right|_{\partial \Omega}$ on $\partial \Omega$, where $\left.\varphi\right|_{\partial \Omega}$ is the trace in $H^{\frac{1}{2}}(\partial \Omega)$ of $\varphi \in H^{1}(\Omega)$, and $\int_{\Omega} \varepsilon \nabla \widetilde{\varphi} \cdot \nabla \varphi^{*} d \mathbf{x}=-\int_{\Omega} \varepsilon \mathbf{g}_{1} \cdot \nabla \varphi^{*} d \mathbf{x}$, for any $\varphi^{*} \in H_{0}^{1}(\Omega)$. Observe that $\nabla \widetilde{\varphi}+\mathbf{g}_{1}$ is $\varepsilon$-orthogonal to $\nabla H_{0}^{1}(\Omega)$, and therefore $\nabla \widetilde{\varphi}+\mathbf{g}_{1} \in H\left(\operatorname{div}_{\varepsilon} ; \Omega\right)$ (again see [30]). Now, define $\widetilde{\mathbf{u}}_{\mathrm{bc}}=\mathbf{g}_{1}+\nabla \widetilde{\varphi}+\mathbf{w}$. By construction, $\mathbf{n} \times \widetilde{\mathbf{u}}_{\mathrm{bc}}=\mathbf{g}$ on $\partial \Omega$, since $\mathbf{n} \times \nabla \widetilde{\varphi}=\mathbf{n} \times \nabla \varphi$ on $\partial \Omega$, and $\nabla \cdot\left(\varepsilon \widetilde{\mathbf{u}}_{\mathrm{bc}}\right)=0$ in $\Omega$ immediately follows from $\nabla \cdot\left(\varepsilon \nabla \widetilde{\varphi}+\varepsilon \mathbf{g}_{1}\right)=0$ and $\nabla \cdot(\varepsilon \mathbf{w})=0$. Therefore, the tangential trace operator is linear continuous and surjective from the subspace $H(\operatorname{curl} ; \Omega) \cap H\left(\operatorname{div}_{\varepsilon}^{0} ; \Omega\right)$ of $H(\operatorname{curl} ; \Omega)$ onto $\mathcal{H}(\partial \Omega)$, and then, up to its kernel, its inverse is continuous, due to the open mapping theorem.

Lemma A.3. Let $\Omega$ and $\Omega_{0}$ be as in Proposition A.1 $\widetilde{\mathbf{u}}_{\mathrm{bc}}$ be as in Lemma A.2, and let $\alpha \in \mathbb{R}^{p}$ be the vector with components $\alpha_{j}=\left\langle\left.\varepsilon \widetilde{\mathbf{u}}_{\mathrm{bc}}\right|_{\Omega_{0}} \cdot \mathbf{n}_{0, j}, 1\right\rangle_{\Gamma_{0, j}}, j=$ $1, \ldots, J$. Then there is a function $\widetilde{\mathbf{u}}_{\mathrm{flux}} \in H_{0}\left(\operatorname{curl}^{0} ; \Omega\right) \cap H\left(\operatorname{div}_{\varepsilon}^{0} ; \Omega_{0}\right)$ such that $\left\langle\left.\varepsilon \widetilde{\mathbf{u}}_{\mathrm{flux}}\right|_{\Omega_{0}} \cdot \mathbf{n}_{0, j}, 1\right\rangle_{\Gamma_{0, j}}=\alpha_{j}, j=1, \ldots, J$, and $\left\|\widetilde{\mathbf{u}}_{\mathrm{flux}}\right\|_{0, \Omega} \leq C\left\|\widetilde{\mathbf{u}}_{\mathrm{bc}}\right\|_{0, \Omega}$.

Proof. The conditions $\left\langle\left.\varepsilon \mathbf{u}_{\text {flux }}\right|_{\Omega_{0}} \cdot \mathbf{n}_{0, j}, 1\right\rangle_{\Gamma_{0, j}}=\alpha_{j}, j=1, \ldots, J$, define a unique $\mathbf{u}_{\text {flux }} \in H_{0}\left(\operatorname{curl}^{0} ; \operatorname{div}_{\varepsilon}^{0} ; \Omega_{0}\right)$ satisfying $\left\|\mathbf{u}_{\text {flux }}\right\|_{0, \Omega_{0}} \leq C|\alpha|$ (see, e.g., 30, Theorem 8.4]; the proof of the inequality is immediate). Actually, $\left\|\mathbf{u}_{\mathrm{flux}}\right\|_{0, \Omega_{0}} \leq C\left\|\widetilde{\mathbf{u}}_{\mathrm{bc}}\right\|_{0, \Omega}$. This can be seen in the following way. By definition of dual norms, by the fact that 
$\Gamma_{0, j}$ is a surface without boundary, and by continuity of the normal trace operator from $H\left(\operatorname{div}_{\varepsilon} ; \Omega_{0}\right)$ onto $H^{-\frac{1}{2}}\left(\partial \Omega_{0}\right)$, we have

$$
\begin{aligned}
& \frac{\left|\left\langle\varepsilon \widetilde{\mathbf{u}}_{\mathrm{bc}} \mid \Omega_{0} \cdot \mathbf{n}_{0, j}, 1\right\rangle_{\Gamma_{0, j}}\right|}{\|1\|_{\frac{1}{2}, \Gamma_{0, j}}} \leq \sup _{\varphi \in H^{\frac{1}{2}}\left(\Gamma_{0, j}\right)} \frac{\left|\left\langle\varepsilon \widetilde{\mathbf{u}}_{\mathrm{bc}} \mid \Omega_{0} \cdot \mathbf{n}_{0, j}, \varphi\right\rangle_{\Gamma_{0, j}}\right|}{\|\varphi\|_{\frac{1}{2}, \Gamma_{0, j}}} \\
& =\left\|\left.\varepsilon \widetilde{\mathbf{u}}_{\mathrm{bc}}\right|_{\Omega_{0}} \cdot \mathbf{n}_{0, j}\right\|_{H^{-\frac{1}{2}}\left(\Gamma_{0, j}\right)} \leq\left\|\left.\varepsilon \widetilde{\mathbf{u}}_{\mathrm{bc}}\right|_{\Omega_{0}} \cdot \mathbf{n}\right\|_{H^{-\frac{1}{2}}\left(\partial \Omega_{0}\right)} \\
& \leq C\left\|\widetilde{\mathbf{u}}_{\mathrm{bc}}\right\|_{H\left(\operatorname{div}_{\varepsilon} ; \Omega_{0}\right)}=C\left\|\widetilde{\mathbf{u}}_{\mathrm{bc}}\right\|_{0, \Omega} .
\end{aligned}
$$

Therefore, $|\alpha| \leq C\left\|\widetilde{\mathbf{u}}_{\mathrm{bc}}\right\|_{0, \Omega}$, from where $\left\|\mathbf{u}_{\mathrm{flux}}\right\|_{0, \Omega_{0}} \leq C\left\|\widetilde{\mathbf{u}}_{\mathrm{bc}}\right\|_{0, \Omega}$ follows. Taking the trivial extension $\widetilde{\mathbf{u}}_{\mathrm{flux}}$ of $\mathbf{u}_{\mathrm{flux}}$ to $\Omega$ completes the proof.

Proof of Proposition A.1 It is enough to define $\widetilde{\mathbf{u}}=\widetilde{\mathbf{u}}_{\mathrm{bc}}-\widetilde{\mathbf{u}}_{\mathrm{flux}}$, where $\widetilde{\mathbf{u}}_{\mathrm{bc}}$ and $\widetilde{\mathbf{u}}_{\text {flux }}$ are as in Lemmas $\mathrm{A.2}$ and $\mathrm{A.3}$ respectively.

A.2. Proof of Theorem 2.1. Recalling that $\mathbf{V}=H(\operatorname{curl} ; \Omega) \cap H\left(\operatorname{div}_{\varepsilon} ; \Omega_{0}\right)$, endowed with the norm

$$
\begin{aligned}
\|\mathbf{v}\|_{\mathbf{V}}^{2}=|\omega| & \left\|\sigma_{\vartheta}^{\frac{1}{2}} \mathbf{v}\right\|_{0, \Omega}^{2}+\left\|\mu^{-\frac{1}{2}} \nabla \times \mathbf{v}\right\|_{0, \Omega}^{2} \\
& +\left\|\nu^{\frac{1}{2}} \nabla \cdot(\varepsilon \mathbf{v})\right\|_{0, \Omega_{0}}^{2}+\lambda \sum_{j=1}^{J}\left|\left\langle\left.\varepsilon \mathbf{v}\right|_{\Omega_{0}} \cdot \mathbf{n}_{0, j}, 1\right\rangle_{\Gamma_{0, j}}\right|^{2},
\end{aligned}
$$

we set $\mathbf{V}_{0}=H_{0}(\operatorname{curl} ; \Omega) \cap H_{\text {flux }}\left(\operatorname{div}_{\varepsilon} ; \Omega_{0}\right)$, with $H_{\text {flux }}\left(\operatorname{div}_{\varepsilon} ; \Omega_{0}\right)$ defined in (A.1).

Consider the bilinear form in (2.11)

$$
a(\mathbf{u}, \mathbf{v})=\int_{\Omega} \mu^{-1} \nabla \times \mathbf{u} \cdot \nabla \times \overline{\mathbf{v}} d \mathbf{x}+i \omega \int_{\Omega} \sigma \mathbf{u} \cdot \overline{\mathbf{v}} d \mathbf{x}+\int_{\Omega_{0}} \nu \nabla \cdot(\varepsilon \mathbf{u}) \nabla \cdot(\varepsilon \overline{\mathbf{v}}) d \mathbf{x} .
$$

In the following proposition, we prove $\mathbf{V}$-ellipticity of $a(\cdot, \cdot): \mathbf{V}_{0} \times \mathbf{V}_{0} \rightarrow \mathbb{R}$. The proof essentially follows the lines of [2, Theorem 3.1], but uses the result of Proposition A.1.

Proposition A.4. There is $C>0$ such that $a(\mathbf{u}, \mathbf{u}) \geq C\|\mathbf{u}\|_{\mathbf{V}}^{2}$ for all $\mathbf{u} \in \mathbf{V}_{0}$.

Proof. Since, for $\mathbf{u} \in \mathbf{V}_{0}, a(\mathbf{u}, \mathbf{u})$ differs from $\|\mathbf{u}\|_{\mathbf{V}}^{2}$ only in that it does not contain $|\omega|\left\|\vartheta^{\frac{1}{2}} \mathbf{u}\right\|_{0, \Omega_{0}}^{2}$, it is enough to prove that, for all $\mathbf{u} \in \mathbf{V}_{0}$,

$$
\|\mathbf{u}\|_{0, \Omega_{0}}^{2} \leq C a(\mathbf{u}, \mathbf{u}) .
$$

In order to do this, we need to establish the existence of a continuous lifting of tangential traces on $\Gamma=\partial \Omega_{\sigma} \cap \partial \Omega_{0}$ with zero divergence and flux conditions. To this end, we introduce the space $H_{0, \Gamma_{-}}\left(\operatorname{curl} ; \Omega_{0}\right)=\left\{\mathbf{v} \in H\left(\operatorname{curl} ; \Omega_{0}\right): \mathbf{n}_{0} \times\left.\mathbf{v}\right|_{\Gamma_{-}}=\right.$ 0 in $\left.H_{00}^{-\frac{1}{2}}\left(\Gamma_{-}\right)\right\}$, where $\Gamma_{-}=\partial \Omega_{0} \backslash \bar{\Gamma}$, i.e., $\Gamma_{-}$is the part of $\partial \Omega_{0}$ contained in $\partial \Omega$. For the definition of $H_{00}^{-\frac{1}{2}}\left(\Gamma_{-}\right)$, see, e.g., [35]. Let $\gamma^{-}$be the restriction of the tangential trace operator to $\bar{\Gamma}$ and $\mathcal{H}(\Gamma)$ its image. A complete characterization of this space, as well as the precise definition of its norm, can be found in [15]. Fix $\mathbf{v} \in H_{0, \Gamma_{-}}\left(\operatorname{curl} ; \Omega_{0}\right)$. According to Proposition A.1 with $\Omega=\Omega_{0}$, let $\mathbf{v}_{\mathrm{bc}} \in$ $H\left(\operatorname{curl} ; \Omega_{0}\right) \cap H_{\text {flux }}\left(\operatorname{div}_{\varepsilon}^{0} ; \Omega_{0}\right)$ be the lifting of $\mathbf{n}_{0} \times \mathbf{v}$ on $\partial \Omega_{0}$, i.e., $\mathbf{n}_{0} \times \mathbf{v}=\mathbf{n}_{0} \times \mathbf{v}_{\mathrm{bc}}$ on $\partial \Omega_{0}$. Then, we also have $\mathbf{v}_{\mathrm{bc}} \in H_{0, \Gamma_{-}}\left(\operatorname{curl} ; \Omega_{0}\right)$ and $\gamma^{-} \mathbf{v}_{\mathrm{bc}}=\gamma^{-} \mathbf{v}$. This shows that $\gamma^{-}$is linear continuous (see [16] and [14]) and surjective from $H_{0, \Gamma_{-}}\left(\operatorname{curl} ; \Omega_{0}\right) \cap$ $H_{\text {flux }}\left(\operatorname{div}_{\varepsilon}^{0} ; \Omega_{0}\right)$ onto $\mathcal{H}(\Gamma)$, and then, up to its kernel, its inverse is continuous, owing to the open mapping theorem. 
Now, let $\mathbf{u} \in \mathbf{V}_{0}$, and denote by $\mathbf{u}_{0}$ the restriction of $\mathbf{u}$ to $\Omega_{0}$. Then, due to the previous considerations, $\mathbf{u}_{0}$ can be decomposition as $\mathbf{u}_{0}=\mathbf{w}+\mathbf{u}_{\mathrm{bc}}$, where $\mathbf{w} \in H_{0}\left(\operatorname{curl} ; \Omega_{0}\right) \cap H_{\text {flux }}\left(\operatorname{div}_{\varepsilon} ; \Omega_{0}\right)$ and $\mathbf{u}_{\mathrm{bc}}$ is such that $\mathbf{u}_{\mathrm{bc}} \in H_{0, \Gamma_{-}}\left(\operatorname{curl} ; \Omega_{0}\right) \cap$ $H_{\text {flux }}\left(\operatorname{div}_{\varepsilon}^{0} ; \Omega_{0}\right), \mathbf{n}_{0} \times \mathbf{u}_{\mathrm{bc}}=\mathbf{n}_{0} \times \mathbf{u}_{0}$ on $\Gamma$ and $\left\|\mathbf{u}_{\mathrm{bc}}\right\|_{H\left(\operatorname{curl} ; \Omega_{0}\right)} \leq C\left\|\gamma^{-} \mathbf{u}\right\|_{\mathcal{H}(\Gamma)}$. From 4, Corollary 3.19], we have

$$
\|\mathbf{w}\|_{0, \Omega_{0}} \leq C\left(\|\nabla \times \mathbf{w}\|_{0, \Omega_{0}}+\|\nabla \cdot(\varepsilon \mathbf{w})\|_{0, \Omega_{0}}\right) \leq C a(\mathbf{u}, \mathbf{u})^{\frac{1}{2}} .
$$

As far as $\mathbf{u}_{\mathrm{bc}}$ is concerned, we can obtain a bound in terms of $a(\mathbf{u}, \mathbf{u})$ as follows. Since $\mathbf{u} \in H(\operatorname{curl} ; \Omega)$, we have that $\mathbf{n}_{0} \times \mathbf{u}_{0}=\mathbf{n}_{0} \times\left.\mathbf{u}\right|_{\Omega_{\sigma}}$ on $\Gamma$. This implies that $\left\|\gamma^{-} \mathbf{u}_{0}\right\|_{\mathcal{H}(\Gamma)}=\left\|\widetilde{\gamma}^{-} \mathbf{u}\right\|_{\mathcal{H}(\Gamma)}$, where $\widetilde{\gamma}^{-}$is the restriction to $\Gamma$ of the tangential trace operator taken from $\Omega_{\sigma}=\Omega \backslash \Omega_{0}$. By the continuity of $\widetilde{\gamma}^{-}$, we conclude that

$$
\left\|\mathbf{u}_{\mathrm{bc}}\right\|_{H\left(\operatorname{curl} ; \Omega_{0}\right)} \leq C\|\mathbf{u}\|_{H\left(\operatorname{curl} ; \Omega_{\sigma}\right)} \leq C a(\mathbf{u}, \mathbf{u})^{\frac{1}{2}} .
$$

This, together with (A.3), implies A.2 and finishes the proof.

Well-posedness of formulation (2.11) can now be proved in a standard way as follows. Let $\mathbf{u}_{f}$ be the (unique) function in $H_{0}\left(\operatorname{curl}^{0}, \operatorname{div}_{\varepsilon}^{0} ; \Omega_{0}\right)$ such that $\left\langle\left.\varepsilon \mathbf{u}_{f}\right|_{\Omega_{0}} \cdot \mathbf{n}_{0, j}, 1\right\rangle_{\Gamma_{0, j}}=\lambda^{-1} f_{j}, j=1, \ldots, J$ (see [30, Theorem 8.4]), and define $\widetilde{\mathbf{u}}_{f}$ as the trivial extension of $\mathbf{u}_{f}$ to $\Omega$. By the decomposition $\varepsilon^{-1} \mathbf{F}=\mathbf{F}^{\prime}+\mathbf{F}^{\prime \prime}$, as in (2.6), with $\mathbf{F}^{\prime \prime}=\nabla f$ in $\Omega_{0}$, and the trace theorem in $H^{1}\left(\Omega_{0}\right)$, we have $\left\|\widetilde{\mathbf{u}}_{f}\right\|_{\mathbf{v}} \leq C\|\mathbf{F}\|_{0, \Omega}$.

Furthermore, let $\widetilde{\mathbf{u}} \in H(\operatorname{curl} ; \Omega) \cap H_{\text {flux }}\left(\operatorname{div}_{\varepsilon}^{0} ; \Omega_{0}\right)$ be such that $\mathbf{n} \times \widetilde{\mathbf{u}}=\mathbf{g}$ on $\partial \Omega$ and $\|\widetilde{\mathbf{u}}\|_{H(\operatorname{curl} ; \Omega)} \leq C\|\mathbf{g}\|_{\mathcal{H}(\partial \Omega)}$, according to Proposition A.1. By defining $\mathbf{u}_{0}=\mathbf{u}-\widetilde{\mathbf{u}}_{f}-\widetilde{\mathbf{u}} \in \mathbf{V}_{0}$, the variational problem 2.11 can be written: Find $\mathbf{u}_{0} \in \mathbf{V}_{0}$ such that, for any $\mathbf{v} \in \mathbf{V}_{0}$,

$$
a\left(\mathbf{u}_{0}, \mathbf{v}\right)=L(\mathbf{v})
$$

where

$$
L(\mathbf{v})=\int_{\Omega} \mathbf{F} \cdot \overline{\mathbf{v}} d \mathbf{x}-\int_{\Omega} \mu^{-1} \nabla \times \widetilde{\mathbf{u}} \cdot \nabla \times \overline{\mathbf{v}} d \mathbf{x}-i \omega \int_{\Omega} \sigma \widetilde{\mathbf{u}} \cdot \overline{\mathbf{v}} d \mathbf{x} .
$$

From Proposition A.4 the bilinear form $a(\cdot, \cdot): \mathbf{V}_{0} \times \mathbf{V}_{0} \rightarrow \mathbb{R}$ is continuous and V-elliptic. Moreover, the linear functional $L(\cdot): \mathbf{V}_{0} \rightarrow \mathbb{R}$ is continuous in the $\mathbf{V}$ norm. Existence and uniqueness of the solution in $\mathbf{u}_{0} \in \mathbf{V}_{0}$, as well as continuous dependence in the $\mathbf{V}$-norm on the data $\mathbf{F}$ and $\widetilde{\mathbf{u}}$ then follow from the Lax-Milgram lemma. We conclude the existence and uniqueness of the solution $\mathbf{u} \in \mathbf{V}$ and the stability estimate

$$
\|\mathbf{u}\|_{\mathbf{v}} \leq C\|\mathbf{F}\|_{0, \Omega}+C\|\widetilde{\mathbf{u}}\|_{\mathbf{v}}+\left\|\widetilde{\mathbf{u}}_{f}\right\|_{\mathbf{v}} \leq C\|\mathbf{F}\|_{0, \Omega}+C\|\mathbf{g}\|_{\mathcal{H}(\partial \Omega)}
$$

where we used Proposition A.1 and the properties of $\widetilde{\mathbf{u}}_{f}$.

To obtain the equivalence of strong and variational form of our model problem we proceed as follows. Integrating by parts and taking into account the properties of $f$, it is obvious that, if $\mathbf{u}$ solves (2.7)-(2.10), then it also solves (2.11), for any $\mathbf{v} \in \mathbf{V}_{0}$. In order to prove the converse, define

$$
\begin{aligned}
& \mathbf{V}_{1}=\left\{\mathbf{v} \in H_{0}(\operatorname{curl} ; \Omega):\left.\mathbf{v}\right|_{\Omega_{0}} \in \nabla H_{0}^{1}\left(\Omega_{0}\right),\left.\mathbf{v}\right|_{\Omega_{\sigma}}=\mathbf{0}\right\}, \\
& \mathbf{V}_{2}=\left\{\mathbf{v} \in H_{0}(\operatorname{curl} ; \Omega):\left.\mathbf{v}\right|_{\Omega_{0}} \in H_{0}\left(\operatorname{curl}^{0}, \operatorname{div}_{\varepsilon}^{0} ; \Omega_{0}\right),\left.\mathbf{v}\right|_{\Omega_{\sigma}}=\mathbf{0}\right\}, \\
& \mathbf{V}_{3}=\left\{\mathbf{v} \in H_{0}(\operatorname{curl} ; \Omega):\left.\mathbf{v}\right|_{\Omega_{0}} \in H_{\text {flux }}\left(\operatorname{div}_{\varepsilon}^{0} ; \Omega_{0}\right)\right\} .
\end{aligned}
$$


Then

$$
\begin{aligned}
& H_{0}(\operatorname{curl} ; \Omega)=\mathbf{V}_{1} \oplus \mathbf{V}_{2} \oplus \mathbf{V}_{3}, \\
& \mathbf{V}_{0}=\left(\mathbf{V}_{1} \oplus \mathbf{V}_{2}\right) \cap H_{\text {flux }}\left(\operatorname{div}_{\varepsilon} ; \Omega_{0}\right) \oplus \mathbf{V}_{3},
\end{aligned}
$$

where the direct sums are understood with respect to the $\varepsilon$-inner product. For test functions $\mathbf{v} \in \mathbf{V}_{3}$, the variational problem (2.11) becomes

$$
\int_{\Omega} \mu^{-1} \nabla \times \mathbf{u} \cdot \nabla \times \overline{\mathbf{v}} d \mathbf{x}+i \omega \int_{\Omega_{\sigma}} \sigma \mathbf{u} \cdot \overline{\mathbf{v}} d \mathbf{x}=\int_{\Omega} \varepsilon \mathbf{F}^{\prime} \cdot \overline{\mathbf{v}} d \mathbf{x}
$$

recalling that $\varepsilon^{-1} \mathbf{F}=\mathbf{F}^{\prime}+\nabla f$. Since for any $\mathbf{v} \in \mathbf{V}_{1} \oplus \mathbf{V}_{2}, \nabla \times \mathbf{v}=\mathbf{0}$ in $\Omega$, $\mathbf{v}=\mathbf{0}$ in $\Omega_{\sigma}$ and $\mathbf{v}$ is $\varepsilon$-orthogonal to $\mathbf{F}^{\prime}$, owing to decomposition A.4 we can take any $\mathbf{v} \in H_{0}(\operatorname{curl} ; \Omega)$ in A.6) without altering the problem, and obtain that (2.7) is satisfied in the sense of the distributions. Since $\varepsilon \mathbf{F}^{\prime} \in L^{2}(\Omega)^{3}$ and $\sigma \mathbf{u} \in L^{2}(\Omega)^{3}$, we have that $\nabla \times\left(\mu^{-1} \nabla \times \mathbf{u}\right) \in L^{2}(\Omega)^{3}$ and (2.7) holds true almost everywhere.

For test functions $\mathbf{v} \in\left(\mathbf{V}_{1} \oplus \mathbf{V}_{2}\right) \cap H_{\text {flux }}\left(\operatorname{div}_{\varepsilon} ; \Omega_{0}\right)$, (2.11) becomes

$$
\int_{\Omega_{0}} \nu \nabla \cdot(\varepsilon \mathbf{u}) \nabla \cdot(\varepsilon \overline{\mathbf{v}}) d \mathbf{x}=\int_{\Omega_{0}} \nabla f \cdot \varepsilon \overline{\mathbf{v}} d \mathbf{x} .
$$

Recall that $f \in H^{1}\left(\Omega_{0}\right), f=0$ on $\Gamma_{0,0}$ and $f$ constant on each $\Gamma_{0, j}, j=1, \ldots, J$. Integrating the right-hand side by parts and taking into account these properties of $f$ and the flux conditions for $\varepsilon \mathbf{v}$, we have

$$
\int_{\Omega_{0}} \nu \nabla \cdot(\varepsilon \mathbf{u}) \nabla \cdot(\varepsilon \overline{\mathbf{v}}) d \mathbf{x}=-\int_{\Omega_{0}} f \nabla \cdot(\varepsilon \overline{\mathbf{v}}) d \mathbf{x},
$$

for any $\mathbf{v} \in\left(\mathbf{V}_{1} \oplus \mathbf{V}_{2}\right) \cap H_{\text {flux }}\left(\operatorname{div}_{\varepsilon} ; \Omega_{0}\right)$. We can take in (A.8) test functions $\mathbf{v} \in\left(\mathbf{V}_{1} \oplus \mathbf{V}_{2}\right) \cap H\left(\operatorname{div}_{\varepsilon} ; \Omega_{0}\right)$ without altering the problem. In fact, given $\mathbf{v} \in$ $\left(\mathbf{V}_{1} \oplus \mathbf{V}_{2}\right) \cap H\left(\operatorname{div}_{\varepsilon} ; \Omega_{0}\right)$, there is a (unique) $\mathbf{v}_{\beta} \in H_{0}\left(\operatorname{curl}^{0}, \operatorname{div}_{\varepsilon}^{0} ; \Omega_{0}\right)$ such that $\left\langle\left.\varepsilon \mathbf{v}_{\beta}\right|_{\Omega_{0}} \cdot \mathbf{n}_{0, j}, 1\right\rangle_{\Gamma_{0, j}}=\left\langle\left.\varepsilon \mathbf{v}\right|_{\Omega_{0}} \cdot \mathbf{n}_{0, j}, 1\right\rangle_{\Gamma_{0, j}}, j=1, \ldots, J$ (again, see 30]). Naming again $\mathbf{v}_{\beta}$ the trivial extension of $\mathbf{v}_{\beta}$ to $\Omega_{\sigma}$, we have that $\mathbf{v}_{0}=\mathbf{v}-\mathbf{v}_{\beta}$ belongs to $\left(\mathbf{V}_{1} \oplus \mathbf{V}_{2}\right) \cap H_{\text {flux }}\left(\operatorname{div}_{\varepsilon} ; \Omega_{0}\right)$, and $\nabla \cdot \varepsilon \mathbf{v}=\nabla \cdot \varepsilon \mathbf{v}_{0}$ in $\Omega_{0}$. Consequently, equation (A.8) holds true also for $\mathbf{v} \in\left(\mathbf{V}_{1} \oplus \mathbf{V}_{2}\right) \cap H\left(\operatorname{div}_{\varepsilon} ; \Omega_{0}\right)$.

Now, for any $\varphi \in L^{2}\left(\Omega_{0}\right)$, let $\psi$ be the (unique) solution in $H_{0}^{1}\left(\Omega_{0}\right)$ of the problem $\nabla \cdot(\varepsilon \nabla \psi)=\varphi$ in $\Omega_{0}, \psi=0$ on $\partial \Omega_{0}$. Denoting again by $\psi$ the trivial extension of $\psi$ to $\Omega_{\sigma}$, we have that $\nabla \psi$ belong to $H_{0}(\operatorname{curl} ; \Omega) \cap\left(\nabla H_{0}^{1}\left(\Omega_{0}\right) \cap H\left(\operatorname{div}_{\varepsilon} ; \Omega_{0}\right)\right)$, which is contained in $\left(\mathbf{V}_{1} \oplus \mathbf{V}_{2}\right) \cap H\left(\operatorname{div}_{\varepsilon} ; \Omega_{0}\right)$. Then, we can take $\mathbf{v}=\nabla \psi$ as test function in (A.8) and obtain that, for any $\varphi \in L^{2}\left(\Omega_{0}\right), \int_{\Omega_{0}} \nu \nabla \cdot(\varepsilon \mathbf{u}) \bar{\varphi} d \mathbf{x}=-\int_{\Omega_{0}} f \bar{\varphi} d \mathbf{x}$, from which (2.8) follows, along with the regularity property $\left.\nu \nabla \cdot(\varepsilon \mathbf{u})\right|_{\Omega_{0}} \in H^{1}\left(\Omega_{0}\right)$.

This completes the proof of Theorem 2.1

\section{REFERENCES}

1. A. Alonso, A mathematical justification of the low-frequency heterogeneous time-harmonic Maxwell equations, Math. Mod. Meth. Appl. Sci. 9 (1999), 475-489. MR 2000c:78002

2. A. Alonso and A. Valli, A domain decomposition approach for heterogeneous time-harmonic Maxwell equations, Comput. Meth. Appl. Mech. Engrg. 143 (1997), 97-112. MR 98b:78020

3. _ An optimal domain decomposition preconditioner for low-frequency time-harmonic Maxwell equations, Math. Comp. 68 (1999), 607-631. MR 99i:78002

4. C. Amrouche, C. Bernardi, M. Dauge, and V. Girault, Vector potentials in three-dimensional nonsmooth domains, Math. Models Appl. Sci. 21 (1998), 823-864. MR 99e:35037

5. D.N. Arnold, An interior penalty finite element method with discontinuous elements, SIAM J. Numer. Anal. 19 (1982), 742-760. MR 83f:65173 
6. D.N. Arnold, F. Brezzi, B. Cockburn, and L.D. Marini, Unified analysis of discontinuous Galerkin methods for elliptic problems, SIAM J. Numer. Anal. 39 (2001/02), 1749-1779. CMP 2002:09

7. I. Babuška and M. Suri, The hp-version of the finite element method with quasiuniform meshes, Modél. Math. Anal. Numér. 21 (1987), 199-238. MR 88d:65154

8. C.E. Baumann and J.T. Oden, A discontinuous hp-finite element method for convectiondiffusion problems, Comput. Methods Appl. Mech. Engrg. 175 (1999), 311-341. MR 2000d:65171

9. A.S. Bonnet-BenDhia, C. Hazard, and S. Lohrengel, A singular field method for the solution of Maxwell's equations in polyhedral domains, SIAM J. Appl. Math. 59 (1999), 2028-2044. MR 2000g:78032

10. A. Bossavit, A rationale for edge-elements in $3 D$ fields computation, IEEE Trans. on Magnetics 24 (1988), 74-79.

11. _ Computational electromagnetism, Academic Press, San Diego, 1998. MR 99m:78001

12. A. Bossavit, "Hybrid" electric-magnetic methods in eddy-current problems, Comput. Methods Appl. Mech. Engrg. 178 (1999), 383-391. MR 2000h:78006

13. C.F. Bryant, C.R.I. Emson, P. Fernandes, and C.W. Trowbridge, Lorentz gauge eddy current formulations for multiply connected piece-wise homogeneous conductors, COMPEL 17 (1998), 732-740.

14. A. Buffa, Hodge decomposition on the boundary of a polyhedron: the nonsimply connected case, Math. Models Methods Appl. Sci. 11 (2001), pp. 1491-1504.

15. A. Buffa and Jr. P. Ciarlet, On traces for functional spaces related to Maxwell's equations. Part I: An integration by parts formula in Lipschitz polyhedra, Math. Models Appl. Sci. 24 (2001), 9-30. MR 2002b:78024

16. _ On traces for functional spaces related to Maxwell's equations. Part II: Hodge decomposition on the boundary of Lipschitz polyhedra and applications, Math. Models Appl. Sci. 24 (2001), 31-48. MR 2002b:78025

17. P. Castillo, Local discontinuous Galerkin methods for convection-diffusion and elliptic problems, Ph.D. thesis, University of Minnesota, Minneapolis, MN, 2001.

18. P. Castillo, Performance of discontinuous Galerkin methods for elliptic partial differential equations, Tech. Report 1764, IMA, University of Minnesota, 2001.

19. P. Castillo, B. Cockburn, I. Perugia, and D. Schötzau, An a priori error analysis of the local discontinuous Galerkin method for elliptic problems, SIAM J. Numer. Anal. 38 (2000), 1676-1706. CMP 2001:08

20. P. Castillo, B. Cockburn, D. Schötzau, and C. Schwab, Optimal a priori error estimates for the hp-version of the local discontinuous Galerkin method for convection-diffusion problems, Math. Comp. 71 (2002), 455-478. CMP 2002:09

21. Z. Chen, Q. Du, and J. Zou, Finite element methods with matching and nonmatching meshes for Maxwell equations with discontinuous coefficients, SIAM J. Numer. Anal. 37 (2000), 15421570. MR 2001h:78044

22. P. Ciarlet, The finite element method for elliptic problems, North Holland, 1978. MR 58:25001

23. B. Cockburn and C. Dawson, Some extensions of the local discontinuous Galerkin method for convection-diffusion equations in multidimensions, The Proceedings of the 10th Conference on the Mathematics of Finite Elements and Applications: MAFELAP X (J. Whitemann, ed.), Elsevier, 2000, pp. 225-238. MR 2001j:65142

24. B. Cockburn, G. Kanschat, I. Perugia, and D. Schötzau, Superconvergence of the local discontinuous Galerkin method for elliptic problems on Cartesian grids, SIAM J. Numer. Anal. 39 (2001), 264-285.

25. B. Cockburn and C.-W. Shu, The local discontinuous Galerkin method for time-dependent convection-diffusion systems, SIAM J. Numer. Anal. 35 (1998), 2440-2463. MR 99j:65163

26. _ Runge-Kutta discontinuous Galerkin methods for convection-dominated problems, J. Sci. Comput. 16 (2001), 173-261.

27. M. Costabel, M. Dauge, and S. Nicaise, Singularities of Maxwell interface problems, Modél. Math. Anal. Numér. 33 (1999), 627-649. MR 2001g:78005

28. M. Dauge, M. Costabel, and D. Martin, Weighted regularization of Maxwell equations in polyhedral domains, Preprint, University of Rennes, 2001. 
29. L. Demkowicz and L. Vardapetyan, Modeling of electromagnetic absorption/scattering problems using hp-adaptive finite elements, Comput. Methods Appl. Mech. Engrg. 152 (1998), 103-124. MR 99b:78003

30. P. Fernandes and G. Gilardi, Magnetostatic and electrostatic problems in inhomogeneous anisotropic media with irregular boundary and mixed boundary conditions, Math. Models Methods Appl. Sci. 7 (1997), 957-991. MR 98h:78006

31. P. Fernandes and I. Perugia, Vector potential formulation for magnetostatics and modeling of permanent magnets, IMA J. Appl. Math. 66 (2001), 293-318. MR 2002e:78003

32. E.H. Georgoulis and E. Süli, hp-DGFEM on shape-irregular meshes: reaction-diffusion, Tech. Report NA 01-09, Oxford University Computing Laboratory, 2001.

33. R. Hiptmair, Symmetric coupling for eddy-current problems, Tech. Report 148, SFB382, Universität Tübingen, 2000.

34. P. Houston, C. Schwab, and E. Süli, Discontinuous hp finite element methods for advectiondiffusion problems, SIAM J. Numer. Anal., to appear.

35. J. L. Lions and E. Magenes, Problèmes aux limites nonhomogènes et applications, Dunod, Paris, 1968. MR 40:512. MR 40:513

36. P. Monk, A finite element method for approximating the time-harmonic Maxwell equations, Numer. Math. 63 (1992), 243-261. MR 94b:65134

37. J. C. Nédélec, Mixed finite elements in $\mathbb{R}^{3}$, Numer. Math. 35 (1980), 315-341. MR 81k:65125

38. Éléments finis mixtes incompressibles pour l'équation de Stokes dans $\mathbb{R}^{3}$, Numer. Math. 39 (1982), 97-112. MR 83g:65111

39. J.T. Oden, I. Babuška, and C.E. Baumann, A discontinuous hp finite element method for diffusion problems, J. Comput. Phys. 146 (1998), 491-519. MR 99m:65173

40. I. Perugia and D. Schötzau, On the coupling of local discontinuous Galerkin and conforming finite element methods, J. Sci. Comp. (2001), no. 16, 411-433.

41. S. Prudhomme, F. Pascal, J.T. Oden, and A. Romkes, Review of a priori error estimation for discontinuous Galerkin methods, Tech. Report 2000-27, TICAM, University of Texas at Austin, 2000.

42. B. Rivière and M.F. Wheeler, A discontinuous Galerkin method applied to nonlinear parabolic equations, Discontinuous Galerkin Methods: Theory, Computation and Applications (B. Cockburn, G.E. Karniadakis, and C.-W. Shu, eds.), Lect. Notes Comput. Sci. Eng., vol. 11, Springer-Verlag, 2000, pp. 231-244. MR 2002d:65105

43. B. Rivière, M.F. Wheeler, and V. Girault, Improved energy estimates for interior penalty, constrained and discontinuous Galerkin methods for elliptic problems, Part I, Computational Geosciences 3 (4) (1999), 337-360. MR 2001d:65145

44. C. Schwab, $p$ - and hp-FEM-Theory and application to solid and fluid mechanics, Oxford University Press, Oxford, 1998. MR 2000d:65003

45. L. Vardapetyan and L. Demkowicz, hp-adaptive finite elements in electromagnetics, Comput. Methods Appl. Mech. Engrg. 152 (1998), 103-124. MR 99b:78003

Dipartimento di Matematica, Università di Pavia, Via Ferrata 1, 27100 Pavia, Italy

E-mail address: perugia@dimat.unipv.it

Department of Mathematics, University of Basel, Rheinsprung 21, CH-4051 Basel, SWITZERLAND

E-mail address: schotzau@math.unibas.ch 